\title{
Nuclear star clusters
}

\section{Nadine Neumayer ${ }^{1}$ (D) Anil Seth ${ }^{2}$ (D) Torsten Böker $^{3}$}

Received: 13 December 2019/Published online: 6 July 2020

(C) The Author(s) 2020

\begin{abstract}
We review the current knowledge about nuclear star clusters (NSCs), the spectacularly dense and massive assemblies of stars found at the centers of most galaxies. Recent observational and theoretical works suggest that many NSC properties, including their masses, densities, and stellar populations, vary with the properties of their host galaxies. Understanding the formation, growth, and ultimate fate of NSCs, therefore, is crucial for a complete picture of galaxy evolution. Throughout the review, we attempt to combine and distill the available evidence into a coherent picture of NSC evolution. Combined, this evidence points to a clear transition mass in galaxies of $\sim 10^{9} M_{\odot}$ where the characteristics of nuclear star clusters change. We argue that at lower masses, NSCs are formed primarily from globular clusters that inspiral into the center of the galaxy, while at higher masses, star formation within the nucleus forms the bulk of the NSC. We also discuss the co-existence of NSCs and central black holes, and how their growth may be linked. The extreme densities of NSCs and their interaction with massive black holes lead to a wide range of unique phenomena including tidal disruption and gravitational-wave events. Finally, we review the evidence that many NSCs end up in the halos of massive galaxies stripped of the stars that surrounded them, thus providing valuable tracers of the galaxies' accretion histories.
\end{abstract}

Nadine Neumayer

neumayer@mpia.de

Anil Seth

aseth@astro.utah.edu

Torsten Böker

tboeker@cosmos.esa.int

1 Max Planck Institute for Astronomy, Königstuhl 17, 69117 Heidelberg, Germany

2 Department of Physics and Astronomy, University of Utah, 115 South 1400 East, Salt Lake City, UT 84112, USA

3 European Space Agency, c/o STScI, 3700 San Martin Drive, Baltimore, MD 21218, USA 


\section{Contents}

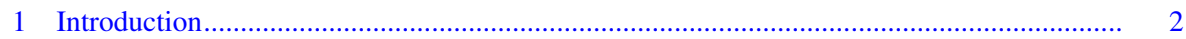

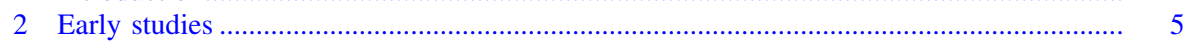

2.1 Imaging nuclear star clusters: the Hubble Space Telescope ..................................... 6

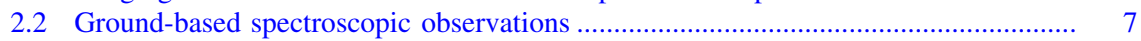

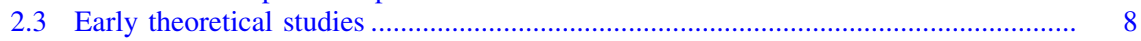

3 What is a nuclear star cluster?.............................................................................. 9

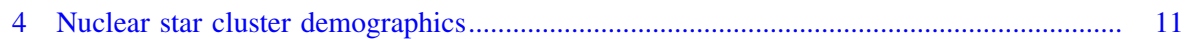

4.1 Trends with host galaxy mass, morphology, and color ...................................... 12

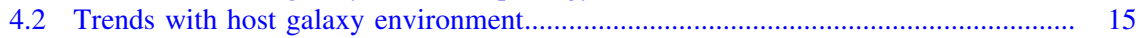

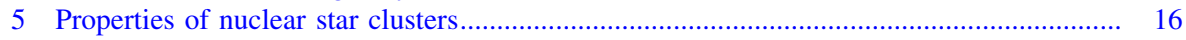

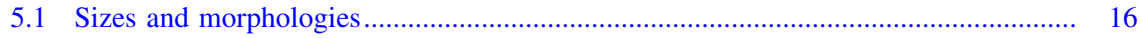

5.2 Luminosities and stellar masses .................................................................. 18

5.3 Size-mass relation and stellar densities........................................................ 19

5.4 Stellar ages and metallicities......................................................................... 21

5.5 Kinematics of NSCs ....................................................................................... 24

5.6 Properties of the Milky Way nuclear star cluster .................................................. 25

5.7 Properties of the M 31 nuclear star cluster ....................................................... 27

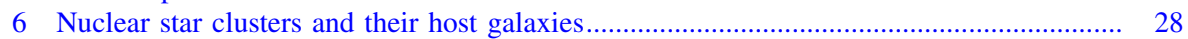

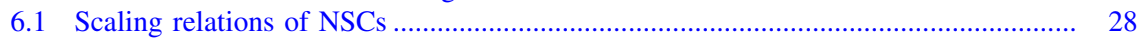

6.2 Other correlations between NSCs and their hosts............................................ 30

7 Scenarios for the formation and growth of nuclear star clusters ............................... 31

7.1 Initial formation of NSCs............................................................................. 31

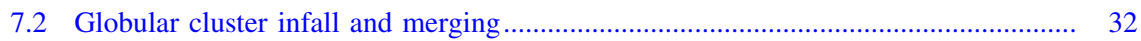

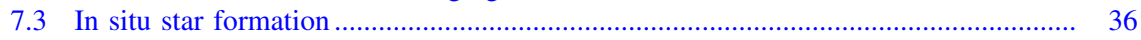

7.4 NSC formation: different galaxies and different mechanisms.............................. 38

8 Nuclear star clusters and massive black holes ....................................................... 40

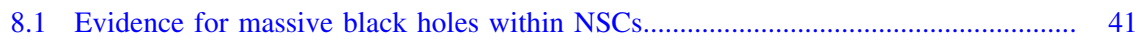

8.2 Co-existence of NSCs and massive black holes ............................................... 41

8.3 Massive black hole formation and growth within NSCs ...................................... 44

9 Stripped down: connecting nuclear star clusters, globular clusters, and ultra-compact dwarf

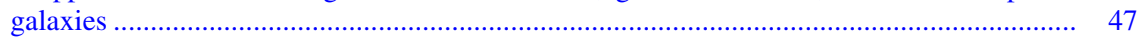

9.1 Extragalactic evidence for stripped NSCs: ultra-compact dwarfs ........................ 47

9.2 Abundance of stripped NSCs ......................................................................... 48

9.3 Stripped NSCs amongst Milky Way globular clusters .............................................. 49

10 The unique astrophysics of very dense stellar systems ........................................... 50

10.1 Stellar dynamical evolution 0

10.2 Nuclear star formation and stellar collisions ....................................................... 51

10.3 Tidal disruption events and SMBH growth................................................... 51

10.4 NSCs as gravitational-wave emitters ............................................................ 52

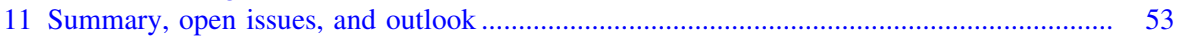

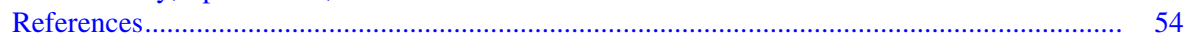

\section{Introduction}

Nuclear star clusters (NSCs) are extremely dense and massive star clusters occupying the innermost region or 'nucleus' of most galaxies. Observationally, NSCs are identified as luminous and compact sources that clearly 'stand out' above their surroundings. This is illustrated in Fig. 1 which shows images and surface 

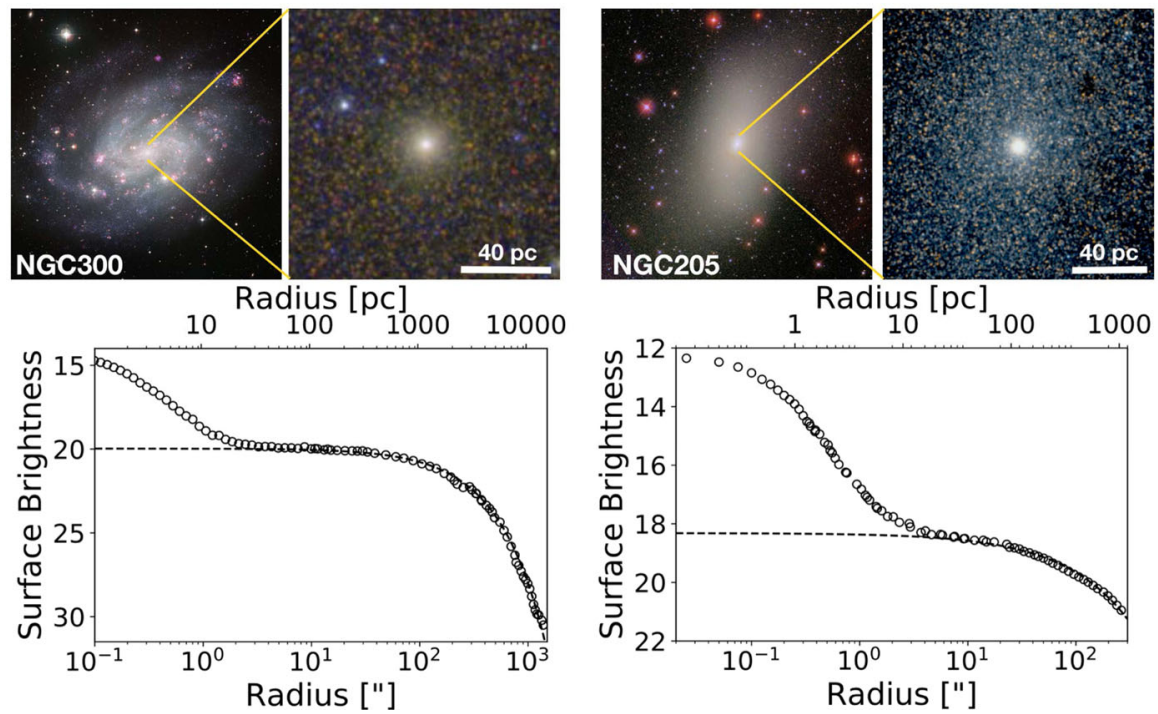

Fig. 1 The NSCs in the late-type spiral NGC 300 (left) and early-type galaxy NGC 205 (M 110, right). The top panels show galaxy-wide images with zoom-ins into the central regions of each galaxy. The bottom panels show surface brightness profiles of the two galaxies, which, in both cases, indicate the presence of an NSC by a rise above the light profile of the host galaxy body. Measured data (in units of $I$ band $\mathrm{mag} / \operatorname{arcsec}^{2}$ ) are shown with open circles, while the dashed line shows a fit to the galaxy profile outside of the NSC proper (an exponential in NGC 300 and a $n=1.4$ Sérsic function in NGC 205; data sources: Böker et al. 2002; Kim et al. 2004; Bland-Hawthorn et al. 2005; Valluri et al. 2005). The largescale image of NGC 300 is from https://www.eso.org/public/images/eso1037a/, while the inset HST image is from Carson et al. (2015). The large-scale image of NGC 205 is from Sloan imaging (retrieved from https://cseligman.com/text/atlas/ngc2.htm), while the inset HST image is from https://www.nasa. gov/feature/goddard/2018/messier-110

brightness profiles of two nearby NSCs in galaxies with very different morphologies: NGC 300 is a disk-dominated late-type spiral without any discernible bulge component, while NGC 205 is a low-mass early-type galaxy with only a minimal amount of gas and recent star formation. The comparison demonstrates that NSCs exist in very different host environments, which raises the question of whether NSC formation and evolution are governed by similar processes in all galaxy types, or whether NSCs follow evolutionary paths that depend on the properties of their host galaxy.

Providing an answer to this question is the goal of a very active research field. To date, more than 400 refereed articles have been written on NSCs, and the pace of discovery about these objects is accelerating, with $\sim 100$ articles appearing in the past 3 years alone. The observational studies cover a wide range of topics, from measuring detailed properties of nearby NSCs (including the one in the Milky Way) to identifying large samples of nuclear star clusters in more distant galaxies of various morphological types. The literature also contains a large number of theoretical studies discussing the two main mechanisms for NSC formation that have been identified so far: the inward migration of star clusters on the one hand, and in situ star formation triggered by high gas densities in the galaxy nucleus on 
the other. Other theoretical papers are focused on the unique and exotic events that can only occur in galaxy nuclei, e.g., tidal disruption events during which a supermassive black hole tears apart one of the stars of the nuclear star cluster, or gravitational-wave emission by mergers of binary black holes.

Despite (or maybe because of) the vast amount of information available, a comprehensive compilation of our current knowledge is lacking, and a literature review that puts NSC properties in the context of their host galaxies is overdue. Specifically, we aim to shed light on the following three questions:

- How do the stars of the NSC get to the nucleus?

- When do they get there, i.e., at what point in the evolution of the host galaxy?

- Are the answers to these questions the same across different galaxy masses and morphologies?

As will become clear, NSCs are very different from other massive star clusters, the properties of which have recently been reviewed by Krumholz et al. (2019) who explicitly exclude NSCs. We also note that the central star cluster of the Milky Way, the closest and best studied example for a typical NSC, will be discussed in some detail in Sect. 5.6 and whenever relevant in the context of the wider family of NSCs. However, we do not provide a comprehensive literature discussion for this object, as it is extensively covered by other recent reviews (Genzel et al. 2010; Schödel et al. 2014b; Bland-Hawthorn and Gerhard 2016). We also point out that the black hole review of Kormendy and Ho (2013) discusses NSCs, especially in the context of how their masses scale with the properties of their host galaxies, a topic we will also discuss in this review. Finally, the formation of NSCs and their relation to ultracompact dwarf galaxies are briefly reviewed by Renaud (2018).

We have organized this review into a number of topical sections: after a short summary of early NSC studies in Sect. 2, we discuss in Sect. 3 how NSCs are identified observationally, including some of the challenges involved. In Sect. 4, we examine how frequently galaxies have NSCs at their centers, and how this varies with galaxy mass and environment. The physical properties of NSCs, including their morphology, masses, densities, stellar populations, and kinematics, are discussed in Sect. 5. We then discuss in Sect. 6 the evidence for any dependencies between NSC properties and those of their host galaxies, focusing, especially, on trends with galaxy stellar mass. Section 7 then discusses different formation scenarios for NSCs, and their implications for the evolution of galactic nuclei. The co-existence of NSCs and massive black holes in galactic nuclei is reviewed in Sect. 8. In Sect. 9, we examine the evidence that stripped NSCs is hidden amongst the populations of globular clusters (GCs) or ultra-compact dwarf galaxies (UCDs). Finally, we discuss the unique dynamics of NSCs in Sect. 10, which can lead to transient tidal disruption and gravitational-wave events. We conclude in Sect. 11 by outlining some open issues and providing an outlook towards future observing facilities which will likely provide important new constraints on the nature of NSCs. We note that the code and data used to make figures in this review is available at https://github.com/anilseth/nsc_review. 


\section{Early studies}

In this section, we provide a short overview of 'historical' (pre-2006) NSC studies. We (somewhat arbitrarily) consider 2006 as an inflection point, because, in that year, three independent studies (Rossa et al. 2006; Wehner and Harris 2006; Ferrarese et al. 2006a) concluded that NSCs are related to their host galaxies in much the same way as SMBHs, which triggered a renewed wave of interest in NSCs and their role in $\mathrm{SMBH}$ growth.

The fact that many galaxies, irrespective of their morphological type, exhibit a prominent and compact surface brightness peak in their morphological center has been known for a long time. Early observations of nearby 'nebula' such as M 31 (Redman and Shirley 1937) or M 33 (Mayall and Aller 1942; Walker 1964) revealed the star-like (i.e., unresolved) surface brightness peak in the nucleus, and even hinted at the presence of young stars and ongoing star formation within it. Later on, high-quality photographic plate catalogs such as those of Sandage (1961) and Sandage and Bedke (1994) clearly showed that the presence of such a bright and unresolved central source is common in nearby galaxies.

For many decades, however, detailed studies of these structures-and the central morphology of external galaxies in general-were difficult or impossible. One important reason is that galactic nuclei were often saturated or 'burned out' in photographic plates, especially in high-surface brightness galaxies. In addition, the seeing-imposed resolution limit of early ground-based observatories smooths out small-scale features in the surface brightness profiles. This makes it difficult to distinguish between a steep, but continuous, rise towards the nucleus on one hand, and a distinctive upturn or 'kink' in the central few parsec on the other. The latter would indicate the presence of a separate morphological component, likely with structural and photometric properties different from those of the surrounding bulge and/or disk.

The first study to unambiguously demonstrate that an extragalactic stellar nucleus is indeed a separate morphological component of its host galaxy was published by Light et al. (1974) who used a stratospheric balloon telescope to improve the resolution of their images of the Andromeda nucleus. Their decomposition of the surface brightness profile in the central arcminute of M 31 led them to conclude that “... the nucleus of M 31 is a distinct and separate feature of that galaxy", albeit without making any statement as to the nature of its emission. Their results motivated the initial theoretical work on NSC formation through dynamical friction by Tremaine et al. (1975). Similar structures were soon confirmed in other nearby galaxies, e.g., M 33 (Gallagher et al. 1982; Nieto and Auriere 1982), M 81, and a handful of others (Kormendy 1985). Other early studies of spiral galaxy (disk) structure such as the one by Romanishin et al. (1983) clearly reveal the ubiquitous presence of unresolved central sources, but do not discuss these in any detail.

As for the Milky Way, the initial detection of its NSC required the development of infrared cameras because of the extreme extinction towards the Galactic center. The first literature reference to the Milky Way NSC was made by Becklin and Neugebauer (1968) who include the following note: "We have observed an 
extended source of infrared radiation which we believe to be at the nucleus of the Galaxy ... We believe that the infrared radiation most likely originates from stars ...". However, resolving individual stars within the Milky Way NSC became possible only several years later (Becklin and Neugebauer 1975).

Around 1980, high-quality photographic plates of nearby galaxy clusters became available which allowed the systematic study of large samples of low-surface brightness galaxies (whose centers were not saturated). Soon after, 'nucleation' was recognized as a common morphological feature of (dwarf) galaxies (e.g., Reaves 1983; Caldwell 1983; Binggeli et al. 1985; Caldwell and Bothun 1987). Initially, these compact central structures were referred to as 'star-like nuclei' or 'semi-stellar nuclei' because of their morphological appearance, i.e., their resemblance to a central bright star. However, it was already suggested by Caldwell (1983) that "Perhaps these nuclei are just tightly bound clusters of stars...", a notion that soon found support from spectroscopic observations. For example, Bothun et al. (1985) concluded that the nuclear emission was produced by a dense accumulation of stars, and so, the term 'stellar nucleus' began to describe both morphology and composition.

The review article by Kormendy and Djorgovski (1989) appears to be the first to clearly equate the terms 'stellar nucleus' and 'nuclear star cluster' while explicitly excluding emission from an active galactic nucleus (AGN). Until recently, the terminology remained somewhat unsettled: 'stellar nucleus' was traditionally used when describing (dwarf) elliptical galaxies, while studies of disk galaxies predominantly used 'nuclear star cluster' (NSC). Most recent publications, however, have adopted the latter term, and we will also use it throughout this review, because it more clearly describes the nature of these objects.

\subsection{Imaging nuclear star clusters: the Hubble Space Telescope}

The rise of digital detectors and the launch of the Hubble Space Telescope (HST) revolutionized the study of NSCs. By the mid-1990s, both ground-based observations using CCDs (e.g., Matthews and Gallagher 1997) and early HST-based studies (Phillips et al. 1996; Sarajedini et al. 1996) had clearly demonstrated that NSCs are much more common than originally thought, and already revealed some early hints at scaling relations between NSC and host galaxy (Phillips et al. 1996). The enormous advance in spatial resolution of the refurbished HST triggered a number of large programs specifically aimed at the nuclear morphology of galaxies. ${ }^{1}$ Much of our current knowledge about NSCs is based on three sets of early HST studies, which were divided by Hubble type and are briefly summarized below.

- A large sample of NSCs in early-type spiral galaxies were observed in the optical (WFPC2, Carollo et al. 1997, 1998; Carollo and Stiavelli 1998), ultraviolet (STIS, Scarlata et al. 2004; Hughes et al. 2005), and infrared (NICMOS,

\footnotetext{
${ }^{1}$ We ignore here the plethora of HST studies aimed at characterizing the AGN phenomenon (Ravindranath et al. 2001). Many of these programs did provide important insight into the circum-nuclear morphology on scales of a few hundred pc, but the presence of a bright AGN usually outshines the stellar light emitted from the central few tens of pc.
} 
Carollo et al. 2002; Seigar et al. 2002). These studies found 'superluminous star clusters' in the nuclei of more than half their sample galaxies, and provided early evidence for scaling relations between the luminosity of NSCs and that of their host galaxies. The latter result was soon refined by Balcells et al. $(2003,2007)$ who pointed out a luminosity correlation between NSC and host galaxy bulge.

- In late-type spirals, the lack of bulges and lower dust content compared to earlytype spirals makes the properties of NSCs easier to study. An early study of 'pure' disk galaxies by Matthews et al. (1999) observed four such galaxies and demonstrated the feasibility to derive structural parameters of their NSCs. A more systematic survey of late-type spirals was performed by Böker et al. (2002) who put on a firm statistical footing the notion that NSCs are ubiquitous even in these dynamically unevolved galaxies.

- In early-type galaxies, Lotz et al. (2001) examined the NSCs in dwarfs in the Virgo and Fornax cluster to test the dynamical friction paradigm for NSC formation. This was followed by the systematic study of Côté et al. (2006) of 100 Virgo cluster galaxies across a wide range of luminosities which provided a clear view of NSC-galaxy scaling relations (Ferrarese et al. 2006a). Their results demonstrated that the fraction of galaxies with NSCs varies with galaxy luminosity, and is nearly $100 \%$ at the low-luminosity end of their sample.

All these studies agree that the vast majority of galaxies with stellar masses $\left(M_{\star}\right)$ between $10^{8}$ and $10^{11} M_{\odot}$ harbour a dominant, compact, and massive stellar cluster in their photometric center (discussed further in Sect. 4). We note that following Bullock and Boylan-Kolchin (2017), when we use the terms "low mass" or "dwarf galaxy" in this review, we refer to galaxies with $M_{\star} \lesssim 10^{9} M_{\odot}$.

\subsection{Ground-based spectroscopic observations}

Given the modest aperture size of HST, its use for spectroscopic NSC studies proves to be challenging. The survey of Rossa et al. (2006), who obtained HST/STIS spectra of $40 \mathrm{NSCs}$ from the Carollo and Böker samples, remains the only large HST-based effort to derive the stellar population of NSCs. While they were able to show that NSCs contain multiple stellar populations, and were among the first $^{2}$ to suggest a correlation between the stellar masses of NSC and host galaxy bulge, the relatively poor signal-to-noise ratio in many of their spectra demonstrates the limited use of HST for NSC spectroscopy.

In general, the superior light-collecting power of large ground-based telescopes is required to obtain deep spectroscopic data of individual NSCs. In low-mass spheroidal galaxies with shallow surface brightness profiles, as well as in bulge-less spiral galaxies with a high nucleus-to-disk contrast, spectroscopy of NSCs is possible even in seeing-limited conditions. This was demonstrated by the work of Bothun et al. (1985) and Bothun and Mould (1988) who concluded that the stellar populations of dE,N nuclei in Virgo have relatively high metallicities, pointing to an

\footnotetext{
${ }^{2}$ Two additional papers (Wehner and Harris 2006; Ferrarese et al. 2006a) arriving at similar conclusions were published in the same year.
} 
extended star formation history. Similarly, Walcher et al. (2006) revealed the presence of young stellar populations in the NSCs of many late-type disk galaxies (see also Sect. 5.4).

In most circumstances, however, seeing-limited observing conditions prevent a clean separation between NSC light and background emission from the host galaxy. Fortunately, over the same time span covered by the HST observations, advances in adaptive optics (AO) technology and laser guide star systems made it possible to overcome these seeing-imposed limitations.

An especially powerful observational technique to study the structure, kinematics, and stellar populations of NSCs is integral-field spectroscopy (IFS). IFS not only allows to probe the extent to which NSCs truly occupy the dynamical center of their hosts, but also to reveal (kinematic and/or stellar population) sub-structures within the NSC, provided that it is nearby enough to be spatially resolved. Some examples for this type of analysis are highlighted in Sect. 5.5. Moreover, when combined with accurate surface brightness profiles, IFS data sets can provide strong constraints on the mass distribution, and in particular the presence of black holes in the mostly unexplored low-mass regime of galaxies with prominent NSCs. This topic is an especially important one which we will discuss further in Sect. 8.

\subsection{Early theoretical studies}

Although Tremaine et al. (1975) had already suggested that NSCs form through the infall and merging of globular clusters driven into the nucleus by dynamical friction, theories for the formation of nucleated low-mass early-type galaxies received renewed attention only following the Virgo cluster study of Binggeli et al. (1985), which identified nucleation as a common feature in many low-mass $\left(M_{\star} \leq 10^{9} M_{\odot}\right)$ early-type galaxies.

The coalescence of globular clusters was further developed analytically by Capuzzo-Dolcetta (1993), as well as by Lotz et al. (2001), who also provided some observational evidence in support of the globular cluster infall scenario. The general validity of dynamical friction as a mechanism for nucleation was confirmed with $\mathrm{N}$ body simulations by Oh and Lin (2000). On the other hand, Milosavljević (2004) concluded that in pure disk galaxies, the migration time scales are too long, and, instead, suggested gas infall and subsequent in situ star formation as the more plausible alternative in disk galaxies.

Other early theoretical studies focused on gas infall and subsequent centralized star formation even in early-type galaxies include Bailey (1980) who argues that inward-flowing stellar mass loss can lead to nuclear stellar disks, and Mihos and Hernquist (1994) who point out that gas-rich mergers will produce dense stellar cores which could be identified as NSCs. In a similar vein, Silk et al. (1987) invoke late gas accretion and the ensuing star formation as a plausible mechanism to explain nucleation, and already point out that this may also explain the higher nucleation rate in more massive dwarf galaxies (see Sect. 4).

We will provide an updated and more detailed summary of NSC formation mechanisms discussed in the recent literature in Sect. 7. 

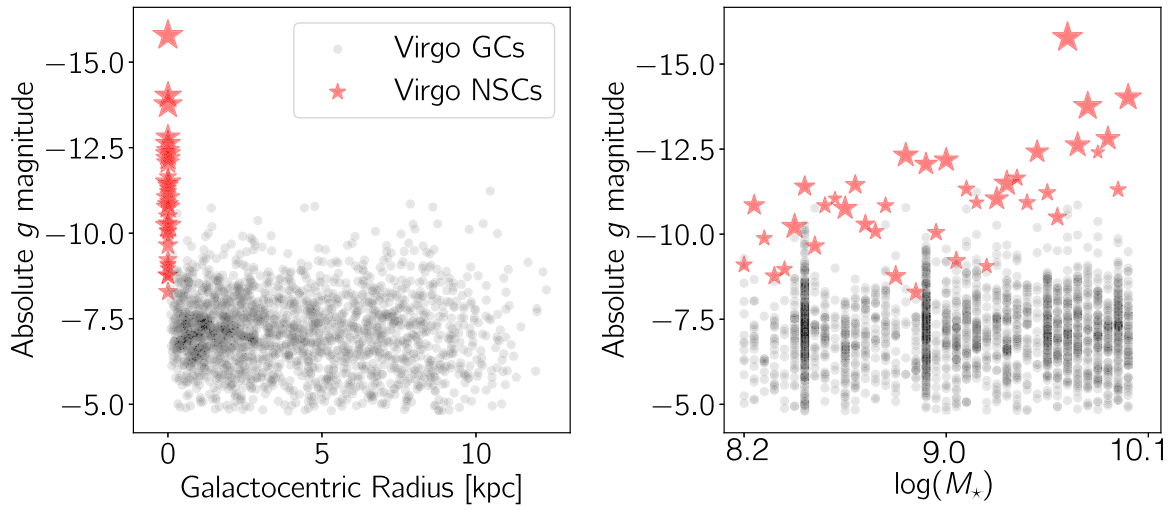

Fig. 2 NSCs (red star symbols) are distinct from globular clusters (GCs, grey points). In both panels, NSCs in the Virgo cluster (Côté et al. 2006) are compared to the GC systems of their hosts, as derived by Jordán et al. (2009). The left panel shows the radial distribution of the clusters vs. their g-band luminosity (note that $M_{g}=-10$ corresponds to a luminosity of $1.1 \times 10^{6} L_{\odot}$ ). The NSCs clearly have exceptional luminosities, and almost all are consistent with being at the photocenter of the galaxy. The right panel shows the distribution of GC and NSC absolute magnitudes separately for each galaxy, and ordered by host stellar mass (from Spengler et al. 2017). We note that, here, the galaxies are evenly spaced in order of increasing stellar mass, and so, their location on the x-axis is only approximate. In both panels, the symbol size for each NSC is proportional to its effective radius, ranging from 2 to $40 \mathrm{pc}$. Note that the increasing symbol size at higher luminosities is a result of a size-luminosity relation for these NSCs; this will be discussed further in Sect. 5.3

\section{What is a nuclear star cluster?}

Given the wealth of observational data on NSCs collected over the past 2 decades, and the wide range of host galaxy properties covered (i.e., Hubble type, stellar masses, environments, star formation activity, etc.), it seems useful to include a clarification of what a nuclear star cluster is.

A broad definition that encompasses the range of objects identified as nuclear star clusters in the literature is the following:

"The detection of stellar excess light above the inward extrapolation of the host galaxy's surface brightness profile on scales of $\lesssim 50$ pc."

While this definition leaves a number of gray areas, in the vast majority of cases, the NSC is unambiguously identified as a dense and massive compact stellar system located at the dynamical center of the host galaxy (e.g., Neumayer et al. 2011), as clearly illustrated in Fig. 1.

In general, NSCs are very distinct, bright, and compact stellar clusters that are unrivalled in luminosity by other stellar clusters. This is clearly demonstrated in Fig. 2 which compares NSCs to the globular cluster systems of their hosts for the Côté et al. (2006) sample of early-type galaxies in Virgo. The right panel shows that independent of host galaxy mass, the NSC is nearly always the brightest cluster in the galaxy, often by a wide margin. This result is remarkable, given that both populations have very similar effective radii (e.g., Böker et al. 2004, see also Sect. 5.1). It seems to hold also for NSC candidates in other types of galaxies such as low-mass dwarfs, as pointed out by Georgiev et al. (2009). Even in the gas-rich 
environment of late-type disk galaxies, the NSC clearly stands out in terms of luminosity, as pointed out by Böker et al. (2002). The only other compact stellar systems rivalling NSCs in luminosity are young and massive 'super star clusters' created in merger events or circum-nuclear starburst rings, which can outshine the NSC for a short time, and in blue passbands.

Despite the prominence of NSCs, there are some complications in identifying them, both observationally and conceptually. These mostly make it difficult to exclude their presence. In what follows, we list some of the challenges and ambiguities in identifying NSCs.

- Steep inner profiles: it can sometimes be difficult to decide what exactly the "inward extrapolation of the host galaxy's surface brightness profile" is. This is especially true in more massive ellipticals and bulge-dominated early-type spirals with steep light profiles (e.g., Carollo et al. 2002; Côté et al. 2007; Kormendy et al. 2009). Galaxies are typically fitted by Sérsic profiles, and Côté et al. (2007) has shown that for a small range of early-type galaxy luminosities around $M_{B}=-20 \mathrm{mag}$, a single Sérsic profile fits the galaxy all the way to the center, while at fainter luminosities, most early-type galaxies show an excess within $0.02 R_{\text {eff }}$ (typically $\lesssim 20 \mathrm{pc}$ ), which they refer to as NSCs. However, this interpretation is somewhat controversial, as Hopkins et al. (2009) and Kormendy et al. (2009) further subdivide this group of lower mass early-type galaxies into ellipticals with central light excess on one hand, and diskier spheroidals with NSCs on the other. ${ }^{3}$ For example, the center of M 32 is described as having a central light excess by Kormendy et al. (2009), but Graham and Spitler (2009) classify it as an NSC with an effective radius of $6 \mathrm{pc}$, well within the distribution of sizes of typical NSCs. In this review, we choose not to make a distinction between NSCs and central light excesses, but will return to this issue when talking about the formation of NSCs in Sect. 7.

- Nuclear disks: flattened structures with disky morphologies are found in massive NSCs in both early-type (Côté et al. 2006; Nguyen et al. 2018) and late-type galaxies including the Milky Way (Seth et al. 2006; Schödel et al. 2014b). These structures show clear evidence for rotation (Seth et al. 2008b; Feldmeier et al. 2014). While the term "nuclear star clusters" may imply spheroidal objects, it is clear that any recent gas infall will likely occur along a preferred plane, and thus, NSC growth via in situ star formation will likely produce a disky morphology. At the same time, many galaxies have larger (circum)nuclear stellar disks on scales of $\approx 100 \mathrm{pc}$ that can be difficult to separate from the NSC proper. For example, the Milky Way has an extended nuclear stellar disk that is clearly distinct from the more compact NSC (Launhardt et al. 2002). On the other hand, some compact nuclear star-forming disks have been identified as a separate class of objects rather than being considered NSCs (e.g., Morelli et al. 2010) despite having scales of only tens of parsecs. Clearly, some ambiguity exists, which has led some authors to exclude large flattened objects from being considered as NSCs (e.g., Scott and Graham 2013). However, we stress that, in

\footnotetext{
3 These papers further argue that light excesses in typically brighter ellipticals and fainter spheroidal systems are not comparable, as they are formed in different processes.
} 
most cases, there is a clear distinction between NSCs (with typical sizes of $5 \mathrm{pc}$ ) and more extended circum-nuclear disks. We note that in both items discussed so far, the ambiguity is primarily present in the nuclei of the most massive galaxies.

- Ongoing starbursts: some galactic nuclei are currently experiencing an episode of active star formation. In these cases, the obscuration by dense clouds of gas and dust, combined with the presence of young stars that dominate the light, can make it difficult to discern the true stellar mass distribution. A good example is NGC 6946 which, despite its proximity, has eluded a characterization of its nuclear morphology (Schinnerer et al. 2006, 2007).

- Ambiguous centers/non-nuclear clusters: for higher mass galaxies with welldefined morphologies and organized rotation, the NSCs appear to be coincident with the photocenter (e.g., Böker et al. 2002; Côté et al. 2006) as well as the dynamical center (Neumayer et al. 2011). However, for many lower luminosity galaxies, the exact location of the galaxy center becomes more ambiguous, especially in irregular galaxies. Nonetheless, the clusters living nearest the centers of these galaxies have similarly exceptional luminosities, suggesting that they are in fact NSCs (Georgiev et al. 2009). Adding to the ambiguity, some star-forming galaxies harbor multiple bright clusters near their centers, making the identification of a bona-fide NSC difficult (e.g., Georgiev and Böker 2014).

- Very low-mass galaxies: the recent discovery of low-mass star clusters $(<$ $6000 \mathrm{M}_{\odot}$ ) near the centers of very faint local group spheroidals (Crnojević et al. 2016; Caldwell et al. 2017) highlights an additional ambiguity. In these galaxies, as well as in somewhat higher luminosity galaxies in Virgo and Fornax (Sánchez-Janssen et al. 2019a; Ordenes-Briceño et al. 2018), the NSCs have lower masses than typical GCs. In contrast to the majority of NSCs, their properties or formation histories may be no different than those of "normal" star clusters.

- Dust lanes: the centers of massive spiral galaxies such as the Milky Way contain dense and thin dust lanes that can make the identification of NSCs challenging. In the Milky Way, the NSC is obscured by $A_{V} \sim 30$ magnitudes (e.g., Fritz et al. 2011; Nogueras-Lara et al. 2019b), making it impossible to identify at optical wavelengths.

To summarize this section, we stress again that in most cases, NSCs are distinct, well-defined objects, even though there are some ambiguous cases, particularly at the extreme ends of the NSC size and mass distribution.

\section{Nuclear star cluster demographics}

In this section, we focus on the question of how frequently NSCs are present in galaxies of different masses and types. Over the last decade, significant additional data sets beyond the initial HST work have been obtained which characterize nuclear star clusters in a wide range of galaxy types and in different environments. 

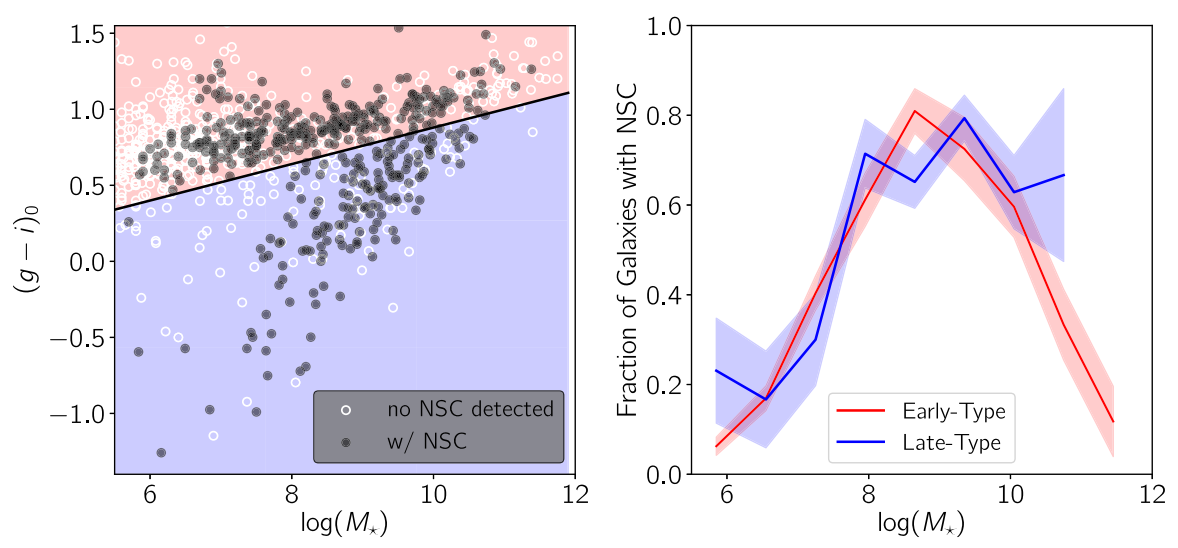

Fig. 3 Nuclear star cluster are found in a wide range of galaxies, but preferentially those with stellar masses between $10^{8}$ and $10^{10} M_{\odot}$. Left: A color-magnitude diagram of galaxies with (filled black circles) and without (open white circles) detected NSCs. The background colors indicate the division between early-type galaxies (the red sequence) and late-type galaxies (the blue cloud). Right: The fraction of galaxies with identified NSCs as a function of stellar mass. Because of various challenges in identifying NSCs (see Sect. 3), these numbers can be considered lower limits for the NSC occupation fraction, especially in late-type galaxies. The data samples for which NSC searches have been conducted (Lauer et al. 2005; Ferrarese et al. 2006b; Georgiev et al. 2009; Georgiev and Böker 2014; Eigenthaler et al. 2018; Sánchez-Janssen et al. 2019a) comprise a total of 1180 galaxies after removal of duplicates. All have available color information that has been transformed to a common color of $(g-i)_{0}$. The values for $M_{\star}$, the stellar masses of the host galaxies, are derived using the Bruzual and Charlot (2003) models with a Chabrier (2003) IMF. Where comparable masses were not listed in the original source, they were calculated using the $g-i$ color-M/L relations of Roediger and Courteau (2015)

A comprehensive list of large NSC studies, which are mostly focused on a specific range of Hubble type, is shown in Table 1.

\subsection{Trends with host galaxy mass, morphology, and color}

We first quantify the fraction of galaxies with NSCs as a function of stellar mass, i.e., the 'NSC occupation fraction'. For early-type galaxies, the recent studies of hundreds of dwarf galaxies in galaxy clusters by den Brok et al. (2014), SánchezJanssen et al. (2019a), and Ordenes-Briceño et al. (2018) have made it clear that NSCs are found in $\gtrsim 80 \%$ of $\sim 10^{9} M_{\odot}$ early-type galaxies, while the NSC occupation fraction drops steadily towards lower host masses, reaching nearly zero at galaxy stellar masses of $\sim 10^{6} M_{\odot}$. These data in clusters also appear to be consistent with the low $<25 \%$ nucleation fraction in early-type dwarf galaxies found around nearby (mostly spiral) galaxies within $12 \mathrm{Mpc}$ by Carlsten et al. (2019). As for the high-mass end, earlier data from the HST surveys of Virgo and Fornax (Côté et al. 2006; Turner et al. 2012) show a decline in the NSC occupation fraction at masses above $10^{9} M_{\odot}$, which is put in context with the lower mass galaxies in the more recent study by Sánchez-Janssen et al. (2019a). In the highest mass ellipticals, NSCs are clearly rarer, but some do appear to exist. For example, Lauer et al. (2005) catalogs a number of nuclear sources with nuclear absorption line spectra and colors similar to the main galaxy, suggesting that they are in fact 
Table 1 NSC literature samples

\begin{tabular}{|c|c|c|c|}
\hline $\begin{array}{l}\text { (1) } \\
\text { Authors }\end{array}$ & $\begin{array}{l}(2) \\
\text { Galaxy sample }\end{array}$ & $\begin{array}{l}\text { (3) } \\
\text { Occ. fraction }\end{array}$ & $\begin{array}{l}\text { (4) } \\
\text { In } \\
\text { Fig. 3? }\end{array}$ \\
\hline $\begin{array}{l}\text { Carollo et al. (1997, 1998, 2002); } \\
\text { Carollo and Stiavelli (1998) }\end{array}$ & $\begin{array}{l}94 \text { early-type spirals (Sa-Sbc) with } \\
\mathrm{v}_{\text {hel }}<2500 \mathrm{~km} / \mathrm{s}, i \leq 75^{\circ} \text {, and } d>1^{\prime}\end{array}$ & $62 \%(58 / 94)$ & No \\
\hline Böker et al. (2002) & 77 face-on late-type spirals (Scd-Sm) & $77 \%(59 / 77)$ & Yes \\
\hline Lauer et al. $(2005)^{\mathrm{a}}$ & $\begin{array}{l}77 \text { massive early-type spirals, } 40 \text { with } \\
\text { color information }\end{array}$ & $20 \%(8 / 40)$ & Yes \\
\hline Côté et al. (2006) & $\begin{array}{l}100 \text { early types in Virgo }(\mathrm{E}, \mathrm{S} 0, \mathrm{dE} \text {, } \\
\mathrm{dS} 0)\end{array}$ & $66 \%(66 / 100)$ & Yes \\
\hline Seth et al. (2006) & 14 edge-on late-type spirals & $64 \%(9 / 14)$ & No \\
\hline Georgiev et al. (2009) & 68 dwarf galaxies (mostly late-type) & $13 \%(9 / 68)$ & Yes \\
\hline Turner et al. (2012) & 43 early types in Fornax & $72 \%(31 / 43)$ & No \\
\hline Georgiev and Böker (2014) & $\begin{array}{l}323 \text { non-active late-type spirals (Sbc- } \\
\mathrm{Sm} \text { ) }\end{array}$ & $\begin{array}{l}71 \%(228 / \\
323)\end{array}$ & Yes \\
\hline den Brok et al. (2014) & 198 early types in Coma & $\begin{array}{l}84 \%(166 / \\
198)\end{array}$ & No \\
\hline Baldassare et al. (2014) & 23 early-type field galaxies & $26 \%(6 / 23)$ & No \\
\hline Ordenes-Briceño et al. (2018) & 225 dwarf galaxies in Fornax & $32 \%(71 / 225)$ & Yes \\
\hline Sánchez-Janssen et al. (2019b) & 404 dwarf galaxies in Virgo & $\begin{array}{l}26 \%(107 / \\
404)\end{array}$ & Yes \\
\hline
\end{tabular}

We note that the sample of Lauer et al. (2005) overlaps with that of Côté et al. (2006) and Turner et al. (2012); a discussion of these overlaps can be found at https://github.com/anilseth/nsc_review. These overlaps have been removed in Fig. 3

${ }^{a}$ We note that the sample of Lauer et al. (2005) overlaps with that of Côté et al. (2006) and Turner et al. (2012); a discussion of these overlaps can be found at https://github.com/anilseth/nsc_review. These overlaps have been removed in Fig. 3

NSCs, although their classification does not universally agree with those of Côté et al. (2006). Counting just these absorption line nuclei as bona-fide NSCs, the nucleation fraction in the Lauer et al. (2005) sample is $20 \%$ (8 out of 40 galaxies with available colors).

As pointed out by Côté et al. (2006), a plausible explanation for the scarcity of NSCs in high-mass ellipticals is the merging of galactic nuclei with SMBHs. This process likely leads to the formation of binary black holes which, in the process of coalescence, transfer energy to the surrounding stars, thus decreasing the central density and effectively destroying the NSC (e.g., Quinlan and Hernquist 1997; Milosavljević and Merritt 2001).

In late-type galaxies, the available data sets are less extensive, with Georgiev and Böker (2014) finding an NSC occupation fraction of $80 \%$ in a sample of over 300 spirals of Hubble-type Sbc and, later, roughly consistent with earlier findings (Böker et al. 2002; Carollo et al. 2002). A much lower NSC occupation fraction is found in a small sample of dwarf galaxies in Georgiev et al. (2009). Similarly, studies of satellite galaxies in Habas et al. (2019) suggest a low occupation fraction in dwarf irregular satellite galaxies in the nearby universe, lower than found for early-type 
galaxies at the same luminosity (but not mass). As discussed in Sect. 3, NSCs can be more challenging to identify in late-type galaxies due to uncertain photocenters, dust obscuration, star formation, and young star clusters that create ambiguity when identifying the NSC. Because of this, the NSC occupation fraction in these studies is really lower limits.

In Fig. 3, we combine the available information on the NSC occupation fraction from all major studies listed above in Table 1. More specifically, we use all objects from the NSC samples of Lauer et al. (2005), Côté et al. (2006), Georgiev et al. (2009), Georgiev and Böker (2014), Ordenes-Briceño et al. (2018), and SánchezJanssen et al. (2019a) for which color information on the host galaxy is available to create the color-mass diagram in the left-hand panel. Galaxies with and without identified NSCs are indicated by filled and open symbols, respectively. Note that the colors and stellar masses of the host galaxies are homogenized to a common photometric system.

In contrast to earlier studies, we here classify NSC host galaxies based on their color rather than their Hubble type, to enable inference of NSC properties in modern large galaxy samples such as those from the Sloan Digital Sky Survey (e.g., Blanton et al. 2005) and future galaxy samples with, e.g., the Large Synoptic Survey Telescope. We use a color cut to divide the galaxy sample into 'blue cloud' and 'red sequence' (corresponding to late and early types, respectively), indicated by the dividing line, as shown in Fig. 3. The amount and uniformity of data on the red sequence is exceptional, with large numbers of galaxies across the full mass range. On the other hand, there is a clear lack of low-mass blue cloud galaxies. This implies that the galaxy samples for which NSC searches have been conducted are significantly biased: at lower masses, the dominant population of galaxies in the universe is actually blue and of late Hubble type (Blanton et al. 2005). We note that the Hubble-type classifications follow closely to the color cut shown here, with just a few galaxies from the early-type samples falling in the blue cloud and vice versa. In the rest of this review, galaxies are divided based on the Hubble type and not based on the color-mass relation shown here due to a lack of available colors and masses for many NSC sample host galaxies.

The right panel of Fig. 3 shows the NSC occupation as a function of galaxy mass, again separated into blue/late-type, and red/early-type galaxies. For the red sequence, this figure is nearly identical to the one shown in Sánchez-Janssen et al. (2019a), although the inclusion of the Lauer et al. (2005) data makes the downturn at the highest masses less pronounced. Remarkably, the blue cloud NSC occupation fraction depends on mass in a way that is very similar to the early types with a peak at $\sim 10^{9} M_{\odot}$ as well. However, note that (1) at low masses, the consistency of early and late types seen here is apparently at odds with initial results from the MATLAS survey where early types have a higher occupation (Habas et al. 2019), and (2) at higher masses, the occupation fraction in late-type galaxies appears to remain high with no clear drop in the highest mass galaxies. This is consistent with the fact that

\footnotetext{
${ }^{4}$ In two cases, the galaxy data are presented in separate papers: Ferrarese et al. (2006b) contains the host galaxy information for the NSC sample of Côté et al. (2006), while Eigenthaler et al. (2018) describes the host galaxy sample of Ordenes-Briceño et al. (2018).
} 
both the Milky Way and Andromeda host prominent NSCs despite having quite high stellar masses. The sample of Carollo et al. (1998) and Carollo et al. (2002) (which are not included in these plots due to a lack of homogeneous galaxy color information) show a nucleation fraction of at least $60 \%$, and given the high masses of these galaxies, this provides further evidence that the highest mass spirals are typically nucleated.

The structural properties of the host galaxies may also affect nucleation. Two recent works have interesting implications for this topic: Lim et al. (2018) find lower surface brightness galaxies to be less frequently nucleated than their higher surface brightness counterparts of the same mass. Moreover, van den Bergh (1986), Ferguson and Sandage (1989), and Lisker et al. (2007) all find that low-mass earlytype galaxies with NSCs are rounder than non-nucleated galaxies of the same galaxy mass. Similarly, den Brok et al. (2015) show that in the Coma cluster, rounder galaxies harbor more massive NSCs than more flattened galaxies of the same stellar mass. Sánchez-Janssen et al. (2019b) find that these results hold in multiple environments, and suggest that nucleation happens preferentially in galaxies that were formed more rapidly, and, thus, have a less flattened stellar mass distribution.

\subsection{Trends with host galaxy environment}

Recently, Sánchez-Janssen et al. (2019a) compared the NSC occupation fraction in lower mass early-type hosts $\left(M_{\star} \lesssim 10^{9} M_{\odot}\right)$ across different galaxy environments. Using the data from den Brok et al. (2014), they found that the NSC occupation fraction is significantly higher in the Coma cluster than in Virgo, with the lowest occupation fractions found in the local group. This conclusion appears to be at odds with a previous study of 28 field ellipticals by Baldassare et al. (2014), who find an NSC occupation fraction similar to that in a mass-matched sample of galaxies in Virgo. We note that a key difference between these two studies is the mass range of galaxies for which the comparison was conducted-the Baldassare et al. (2014) work is focused on high-mass galaxies with $M_{\star}>3 \times 10^{9} M_{\odot}$, while this regime was not probed by the study of Sánchez-Janssen et al. (2019a).

A related observation is the radial distribution of nucleated galaxies in cluster environments, a measurement that has only been done for spheroidal galaxies. Early studies of nucleated early-type dwarfs in Virgo $\left(M_{\star} \lesssim 10^{8} M_{\odot}\right)$ suggested that they were more centrally concentrated within the cluster than non-nucleated galaxies (Ferguson and Sandage 1989). Put differently, the NSC occupation fraction declines at lower galaxy number densities. This result was confirmed in subsequent work by Lisker et al. (2007), and for low-surface brightness galaxies in Coma by Lim et al. (2018). Overall, there is strong evidence that in denser environments, the NSC occupation fraction is enhanced for low-mass early-type galaxies $\left(M_{\star} \lesssim 10^{9} M_{\odot}\right)$. However, the same may not be true for higher host galaxy masses. We note that all of these studies focus on early-type galaxies in cluster environments. The Georgiev et al. (2009) and Georgiev and Böker (2014) samples of late-type galaxies are based on archival data of galaxies, and mostly are in the field, yet their occupation is very high in the mass range between $M_{\star} \sim 10^{8}$ and $10^{10} M_{\odot}$. This would seem to argue 
against the environmental dependence extending across all Hubble types, and perhaps being just restricted to nucleation in early-type galaxies. Careful studies comparing satellites and central galaxies in a range of environments are clearly needed to resolve this issue.

\section{Properties of nuclear star clusters}

Perhaps unsurprisingly given their unique location in the deep potential well of a dark-matter halo, NSCs have extreme properties: they are the brightest, most massive, and densest stellar clusters known. In this section we will review the observational evidence for this statement, as well as the data for other NSC properties such as their stellar composition and kinematics. Finally, we briefly summarize the properties of the NSCs of the Milky Way and M 31, to compare these unique objects to the population of NSCs. We tabulate typical properties of NSCs in different types and masses of galaxies, as well as the Milky Way NSC properties in Table 2.

\subsection{Sizes and morphologies}

The size of an NSC, and in fact any star cluster, is not easily defined, because it depends on the analytic model used to describe the distribution of stars within the cluster. Typically, the metric used is the effective radius $r_{\text {eff }}$ within which half of the cluster light is contained. This measurement is preferred, because it is fairly robust against the details of the model parameters used to fit the NSC profile (Larsen 1999).

Because all but the closest NSCs are barely resolved even with HST, their effective radii are usually measured via PSF-fitting techniques that describe the observed NSC shape as a convolution of the instrumental point spread function (PSF) with an analytical function used to describe Galactic GCs or galaxy light profiles, e.g., the widely used King models (King 1962) or Sérsic profiles (Sérsic 1968; Graham and Driver 2005). This approach has been applied both to onedimensional surface brightness profiles (e.g., Böker et al. 2002; Côté et al. 2006) and two-dimensional images of the NSC (e.g., Böker et al. 2004; Turner et al. 2012; Georgiev and Böker 2014; Spengler et al. 2017). The latter approach has the advantage that one can simultaneously quantify the ellipticity of (well-resolved) NSCs.

The results from the various studies are summarized in Fig. 4, which illustrates that the median size of NSCs $\left(r_{\mathrm{eff}}=3.3_{-1.9}^{+7.0} \mathrm{pc}\right)$ is comparable to that of globular clusters (Harris 1996; McLaughlin and van der Marel 2005), although the distribution has a noticeable tail towards larger sizes.

Figure 5 demonstrates that many NSCs appear non-spherical, with ellipticities as high as 0.6 (ellipticity: $\epsilon=1-b / a, a$ : major axis, and $b$ : minor axis). Reliably measuring the axis ratio $b / a$ of barely resolved sources is difficult, especially in nuclei of spiral galaxies which are often affected by patchy dust extinction. Nevertheless, observations of edge-on spirals by Seth et al. (2006) have clearly 
Table 2 NSC properties

\begin{tabular}{llllll}
\hline$(1)$ & $(2)$ & $(3)$ & $(4)$ & $(5)$ & $(6)$ \\
Galaxy type & $\begin{array}{l}\text { Size } \\
r_{\text {eff }}[\mathrm{pc}]\end{array}$ & $\begin{array}{l}\text { Ellipticity } \\
\epsilon=1-b / a\end{array}$ & $\begin{array}{l}\text { Mass } \\
\log \left(M_{\mathrm{NSC}}\right)\end{array}$ & $\begin{array}{l}\text { Mass fraction } \\
M_{\mathrm{NSC}} / M_{\star}\end{array}$ & $\begin{array}{l}\text { Surface mass density } \\
\Sigma_{\mathrm{eff}}\left[M_{\odot} / \mathrm{pc}^{2}\right]\end{array}$ \\
\hline Early types & & & & \\
$<10^{9} M_{\odot}$ & $4.4_{-1.7}^{+6.5}$ & $0.16_{-0.08}^{+0.06}$ & $5.8_{-0.6}^{+0.6}$ & 0.020 & $10^{4.1}$ \\
$>10^{9} M_{\odot}$ & $4.4_{-1.8}^{+17.9}$ & $0.17_{-0.08}^{+0.20}$ & $7.0_{-0.6}^{+0.8}$ & 0.004 & $10^{4.6}$ \\
Late types & & & & \\
$<10^{9} M_{\odot}$ & $3.1_{-2.0}^{+2.4}$ & $0.37_{-0.22}^{+0.14}$ & $6.2_{-0.5}^{+0.4}$ & 0.007 & $10^{3.6}$ \\
$>10^{9} M_{\odot}$ & $4.8_{-2.5}^{+7.5}$ & $0.20_{-0.09}^{+0.17}$ & $6.8_{-0.8}^{+0.8}$ & 0.001 & $10^{4.7}$ \\
Milky Way & $4.2 \pm 0.4$ & $0.29 \pm 0.02$ & 7.5 & 0.0005 & $10^{5.3}$ (within 4.2 pc) \\
& & & & & $10^{6.4}$ (within 0.5 pc)
\end{tabular}

For all galaxy samples, NSC sizes and surface mass density values are derived from the data shown in Fig. 7, ellipticities from Fig. 5, and the masses and mass fractions from Fig. 12. We note that the samples of early and late-type galaxies differ in their stellar mass distribution, especially at low masses. Values given are medians for the sample, while error bars indicate the 16th and 84th percentiles. For more details on the Milky Way estimates, see Sect. 5.6

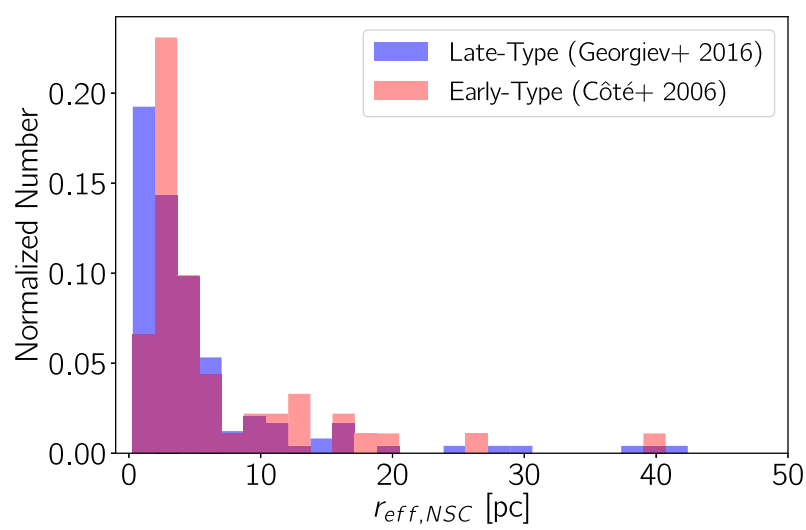

Fig. 4 The effective radii of most NSCs are below $10 \mathrm{pc}$, but a tail of larger NSCs exists in both early and late-type galaxies. Data for early-type galaxies are taken from the ACSVCS survey (Côté et al. 2006), and for late-type spiral galaxies from Georgiev et al. (2016). Note that Côté et al. (2006) resolve effective radii only above $\sim 2 \mathrm{pc}$, but all data have been plotted. For the Georgiev et al. (2016) sample, we exclude objects with only upper limits for $r_{\mathrm{eff}}$, although this has a little impact on the size distribution (because of the wide range of distances in this sample)

identified elongated, disk-like structures in NSCs that are well aligned with the disk of their host galaxies. The results of Spengler et al. (2017) demonstrate that many nuclei in spheroidal galaxies also show a pronounced ellipticity, and that the most elongated ones are also well aligned with their host bodies. Moreover, the amount of ellipticity appears to scale with NSC and host galaxy mass. We will come back to these results in Sect. 7, because they add important constraints on competing formation scenarios for NSCs. 


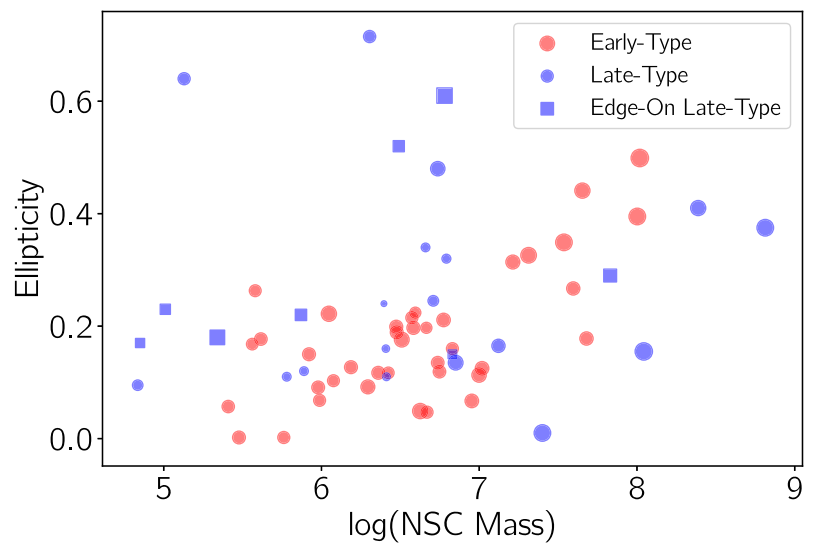

Fig. 5 The highest mass NSCs tend to be more flattened (i.e., have higher ellipticity) than lower mass NSCs. This is especially true in the early-type galaxy sample of Spengler et al. (2017), while late-type galaxies can have very flattened NSCs even in low-mass galaxies. The late-type galaxies plotted contain reliable measurements from Georgiev and Böker (2014) (circles), while squares are edge-on galaxies including the Milky Way (Seth et al. 2006; Schödel et al. 2014a). All symbols are sized based on their effective radius; the range in the effective radii is $0.4-44 \mathrm{pc}$

\subsection{Luminosities and stellar masses}

One of the most directly measurable properties of NSCs is their luminosity. However, aperture photometry cannot usually be applied, as the underlying host galaxy structure is often complex and steeply rising towards the center, and, thus, cannot easily be removed. The preferred method to measure the magnitudes of resolved NSCs, therefore, is to integrate a model parameterization of their surface brightness distribution. This approach has been used both for spiral galaxies (Carollo et al. 2002; Böker et al. 2002; Georgiev and Böker 2014) as well as for early-type samples (Côté et al. 2006; Turner et al. 2012).

The total stellar mass of an NSC is an even more fundamental quantity which, however, is not straightforward to measure. Generally speaking, the mass of a stellar system can be derived via three different observational approaches: (1) photometric, (2) spectroscopic, and (3) dynamical.

Most of the mass measurements for NSCs come from photometric studies, where luminosities are converted into mass using relations between the color and the massto-light ratio of a stellar population, which are selected based on (fits to) the spectral energy distribution (SED) of the stellar population (Georgiev et al. 2016; Spengler et al. 2017; Sánchez-Janssen et al. 2019a). To make this method robust, multi-band photometry is required, with at least one colour measurement to constrain the stellar population. Unfortunately, there is no common set of filters used for all the observations, which adds further complexity.

Stellar population synthesis methods can also be used to estimate the mass-tolight ratio (Rossa et al. 2006; Seth et al. 2010; Kacharov et al. 2018). These massto-light ratios based on full spectrum fitting are more reliable than photometric measurements, especially if those are based on just a single color. Dynamical mass 
measurements are considered the most accurate way to estimate masses. To measure the mass, the mass-to-light ratio of the NSC is determined by comparing the measured (typically integrated) velocity dispersion to a dynamical model for the stellar potential based on the luminosity profile of the NSC. Since the integrated velocity dispersions of NSCs are typically low $(<20-30 \mathrm{~km} / \mathrm{s})$, the spectral resolution of the observations needs to be sufficiently high $(R \gtrsim 5000$, e.g., Walcher et al. 2005; Kormendy et al. 2010). Recently, Nguyen et al. (2019) showed that mass-to-light ratios based on stellar population synthesis vary by less than $10 \%$ with dynamical estimates based on kinematic maps of four NSCs, suggesting that stellar population synthesis also provide quite reliable masses.

Our current knowledge of the NSC mass distribution is summarized in Fig. 6 which demonstrates that NSCs are, on average, much more massive than globular clusters, especially in hosts with masses above $10^{9} M_{\odot}$-we consider the scaling of NSC and galaxy mass further in Sect. 6.1. The high masses of NSCs combined with their similar sizes to GCs (see Sect. 5.1) already suggest that the stellar densities of NSCs are extremely high, a fact that we will discuss next.

\subsection{Size-mass relation and stellar densities}

In Fig. 7, we compare the mass-density and size-mass relations of NSCs to those of other stellar systems. Similar plots have previously been published by various authors (e.g., Hopkins and Quataert 2010b; Misgeld and Hilker 2011; Norris et al. 2014). Our version has a more complete sample of NSCs including recently

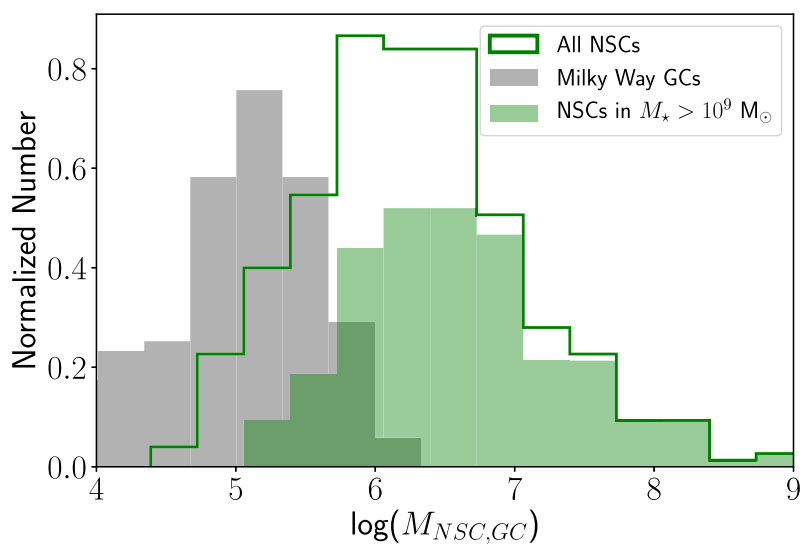

Fig. 6 Mass distribution of NSCs relative to that of globular clusters, showing that NSCs in massive galaxies are typically an order of magnitude more massive. Data for NSCs (open and filled green histograms) are estimated via color $-M / L$ relations or SED fitting to determine their $M / L$, and using the Bruzual and Charlot (2003) models with a Chabrier (2003) IMF. The sample of NSCs includes both earlytype galaxies from the Virgo cluster (Spengler et al. 2017; Sánchez-Janssen et al. 2019a) and late-type galaxies from (Georgiev et al. 2016). The masses of NSCs are very dependent on the host galaxy sample: the filled green histogram only includes host galaxies with masses above $10^{9} \mathrm{M}_{\odot}$, while the open green histogram also includes less massive hosts. The globular cluster masses are taken from the updated Harris catalog (Harris 1996) assuming an $M / L_{V}$ of 2, typical for GCs of all metallicities (e.g., Voggel et al. 2019) 

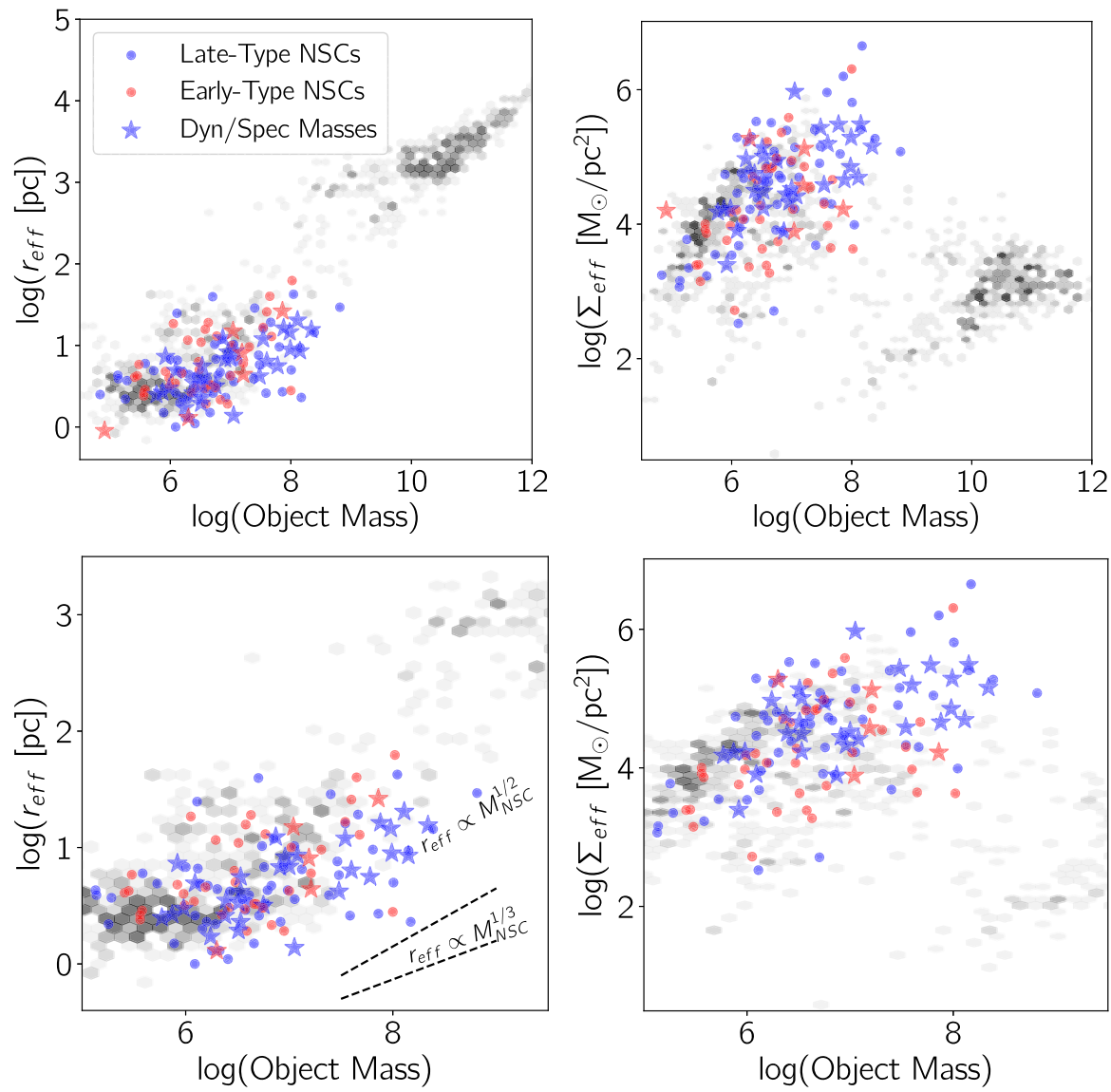

Fig. 7 Mass-radius and mass-surface density relations for NSCs (colored points), compared to other hot dynamical systems (GCs, bulges, elliptical galaxies; grayscale) from Norris et al. (2014). The blue asterisks denote measurements compiled by Erwin and Gadotti (2012), which stem from dynamical measurements or spectral synthesis fits, taken mostly from Walcher et al. (2005) and Rossa et al. (2006). Other NSC data are taken from Georgiev et al. (2016) for late-types, and the ACSVCS for early-types (Côté et al. 2006; Spengler et al. 2017). Other hot stellar systems plotted in gray include globular clusters, ultra-compact dwarfs, compact ellipticals, and normal early-type galaxies. For these, the masses plotted denote the total stellar mass of the compact object/galaxy. The top two panels plot the data over the full mass range of hot stellar systems, while the bottom two panels 'zoom in' to just the region of parameter space covered by NSCs. The dashed lines in the lower left panel are a line of constant surface density (top line) and constant mass density (bottom line). Above a few million solar masses, NSCs show a positive mass-radius relation, which results in a flattening in surface densities with increasing mass

published data, and distinguishes the most reliable NSC dynamical and stellar population synthesis mass measurements (Erwin and Gadotti 2012; Nguyen et al. 2018). We draw three conclusions from Fig. 7 :

1. For NSCs with masses below a few $10^{6} M_{\odot}$, the size of the NSC does not depend on their mass, while for higher masses (above $\sim 10^{7} M_{\odot}$ ), the size appears to increase proportionally to the square root of the mass, thus preserving 
a constant surface density. We note that we have excluded size upper limits in this figure and only plotted NSCs where the effective radius was measured through modeling of the cluster and the PSF.

2. At a given NSC stellar mass, NSCs in early-type galaxies are on average larger than those in late-type galaxies. A factor of two difference was previously noted by Georgiev et al. (2016), but the data shown here suggest that this offset may not extend down to the lowest mass NSCs. Quantitatively, above $10^{7} M_{\odot}$, the median size of NSCs in early-types is $11.4 \mathrm{pc}$, more than double that of the NSCs in late-types (median of $5.1 \mathrm{pc}$ ). However, below $10^{7} M_{\odot}$, the median sizes are 3.3/4.5 pc in early/late-type galaxies.

3. The most massive NSCs are the densest known stellar systems, and can reach mass surface densities of $\sim 10^{6} M_{\odot} / \mathrm{pc}^{2}$ or more. This is true even for reliable (i.e., spectroscopically derived) NSC masses, which suggests that the $\sim 10^{5} M_{\odot} / \mathrm{pc}^{2}$ upper limit in surface density suggested by Hopkins et al. (2010) to be due to stellar feedback may need to be revised upward. These dense massive clusters have masses derived primarily from the stellar population fits of Rossa et al. (2006).

While surface mass densities are available in a wide range of NSCs, estimates of the volume densities of NSCs have been derived in only a handful of systems. Lauer et al. (1998) derived deprojected density profiles in M 31, M 32, and M 33, and found the central densities (on scales of $\sim 0.1 \mathrm{pc}$ ) are $\sim 10^{6} M_{\odot} / \mathrm{pc}^{3}$ for M 31 and M 33 (see also Lauer et al. 1993; Kormendy and McClure 1993) and $\sim 10^{7} M_{\odot} / \mathrm{pc}^{3}$ for M 32, and that the volume density profile of M 32 goes as $\rho(r) \propto r^{-3 / 2}$. Schödel et al. (2018) derived the three-dimensional stellar density for the Milky Way NSC on scales of $0.01 \mathrm{pc}$ to $2.6 \pm 0.3 \times 10^{7} M_{\odot} / \mathrm{pc}^{3}$. A recent study by Pechetti et al. (2019) derived volume density profiles in a number of the nearest NSCs and finds a clear correlation between the galaxy mass and the density and slope of their NSC profiles, with higher mass galaxies having denser NSCs and shallower density profiles. The NSC mass profiles range from $\rho(r) \propto r^{-1}$ to $r^{-3}$ with the densities at $r=5$ pc ranging from $10^{2}$ to $\sim 10^{4} M_{\odot} / \mathrm{pc}^{3}$.

\subsection{Stellar ages and metallicities}

Measuring individual stellar ages and metallicities within an NSC is the most direct way to derive its formation history. Unfortunately, this is an extremely difficult undertaking, because individual stars can only be resolved in a few very nearby NSCs. For most NSCs in external galaxies, we must rely on the analysis of the integrated light of the entire cluster after careful subtraction of the surrounding galaxy light. Population fitting techniques are usually applied to this integrated light data to determine the combination of stellar ages and metallicities that best describe the observed spectral energy distribution. Even with high-quality spectra or multiband images, there are many challenges and limitations associated with stellar population modeling, which have been extensively discussed recently by Conroy (2013). Generally speaking, the youngest population of stars in an NSC can be fairly 
reliably determined, because they dominate the light. On the other hand, the age and mass of the oldest populations (which provides the strongest constraints on when the NSC formed) are extremely challenging to derive. We focus first on late-type galaxies, then early-types, followed by a short section on the nucleus of the Sgr dwarf spheroidal galaxy.

Late-type galaxies: Spectroscopic and multi-band photometric studies of latetype galaxies, for both individual objects (e.g., Böker et al. 1997, 2001; Seth et al. 2006) and larger samples of galaxies (Walcher et al. 2005; Rossa et al. 2006; Carson et al. 2015; Kacharov et al. 2018), all agree that most late-type NSCs contain a mix of stellar populations, with the mass dominated by old stars with ages of more than a few Gyr. In all cases, an extended star formation history provides significantly better fits to the NSC spectra than single stellar populations. This is demonstrated in Fig. 8 for the case of NGC 247. All NSCs in the latest-type spirals appear to contain stars younger than $100 \mathrm{Myr}$ (Walcher et al. 2005; Kacharov et al. 2018). In earlier type spirals, Rossa et al. (2006) find that half of their NSCs have a significant population of stars younger than one Gyr. The Milky Way NSC appears to be a typical example, containing both very young stars and a dominant old population (Blum et al. 2003; Pfuhl et al. 2011, see also Sect. 5.6). A young, 200 Myr old stellar population is also seen at the center of the M 31 NSC (Bender et al. 2005). These young stars are typically centrally concentrated within the NSC (Georgiev and Böker 2014; Carson et al. 2015), although, occasionally, they will be found in a larger disk or ring structure (Seth et al. 2006). As discussed further in Sect. 7, the ubiquitous presence of a young population is strong evidence that NSCs in late-type hosts experience periodic in situ star formation triggered by infalling gas.

Early-type galaxies: The situation is less clear in early-type hosts. The nearest examples seem to have substantial young populations, with ages less than a Gyr

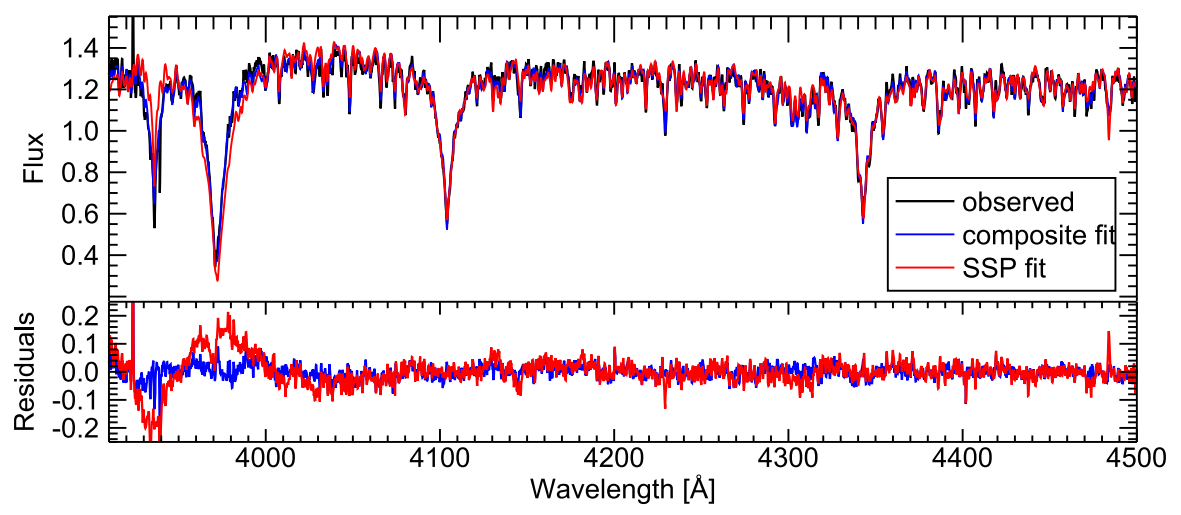

Fig. 8 Stellar population fits for NGC 247 (from Kacharov et al. 2018). The fit residuals are significantly improved when including multiple populations of stars. In this case, the best-fit stellar population model fit (blue line) has $\sim 50 \%$ of the light and $90 \%$ of the mass coming from populations with ages $>1 \mathrm{Gyr}$ and subsolar metallicity, while significant light contributions are also seen from stars of $\sim 10$ and $\sim 100$ Myr, both with slightly super-solar metallicities. The best-fit single stellar population has an age of 0.22 Gyr, and $[\mathrm{Fe} / \mathrm{H}]=+0.26$ 

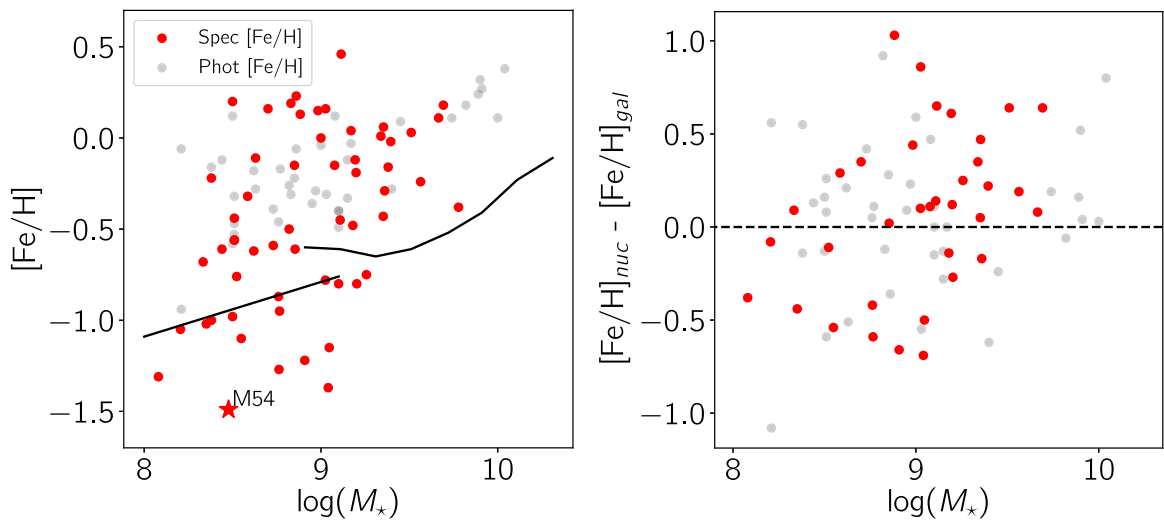

Fig. 9 Metallicity estimates for NSCs in early-type galaxies. Left: NSCs in higher mass galaxies are all metal-rich, while a range of metallicities exist at lower host masses. The spectroscopic data (red symbols) include results from four studies (Koleva et al. 2009; Paudel et al. 2011; Spengler et al. 2017; Kacharov et al. 2018). The photometric metallicity estimates (grey symbols) are from Spengler et al. (2017). The estimate for the nucleus of the Sgr dwarf (labeled M 54) is that of its dominant metal-poor population (e.g., Mucciarelli et al. 2017). M 54 is the only NSC plotted here for which individual stars can be resolved and their abundances determined. The two solid lines are the mass-metallicity relations of Kirby et al. (2013) at the low-mass end, and Gallazzi et al. (2005) at the high mass end. Right: the difference in metallicities between the NSC and their host galaxies. Data are from Koleva et al. (2009); Paudel et al. (2011); Kacharov et al. (2018). Difference values above zero indicate that the NSC is more metal-rich than its host, which predominantly occurs in high-mass galaxies

(Monaco et al. 2009; Kacharov et al. 2018). However, most early-type NSC hosts are found in dense galaxy clusters where typically, star formation has ceased due to the lack of molecular gas. Therefore, most stellar population studies of NSCs in early-type galaxies assume stellar ages above $1 \mathrm{Gyr}$, and use single stellar population models to fit their spectra and spectral energy distributions (e.g., Koleva et al. 2009; Paudel et al. 2011; Spengler et al. 2017). These studies find median spectroscopic ages of NSCs of $\sim 3$ Gyr (Paudel et al. 2011; Spengler et al. 2017). Furthermore, NSCs in early-type galaxies are typically younger than their surrounding galaxy (Koleva et al. 2009; Chilingarian 2009; Paudel et al. 2011). We caution against interpreting these age results too strongly, as the SSP assumptions made by these studies are likely incorrect (see section on M 54 below). Furthermore, the age and metallicity estimates are degenerate, with the age being significantly more uncertain (e.g., Worthey 1994; Spengler et al. 2017).

In Fig. 9, we show metallicities for a sample of NSCs in early-type galaxies. The NSCs with spectroscopically measured metallicities (red symbols; Koleva et al. 2009; Paudel et al. 2011; Spengler et al. 2017) show a fairly clear transition at galaxy stellar masses of $\sim 10^{9} M_{\odot}$. Above this galaxy mass, NSCs are uniformly metal-rich, and fall above the median mass-metallicity relationship, as might be expected due to metallicity gradients within the galaxies (e.g., Koleva et al. 2011). For galaxies with $M_{\star}<10^{9} M_{\odot}$, a much wider range of NSC metallicities is found, with roughly half of the measurements falling on or below the median galaxy massmetallicity relationship. This same trend is seen when directly comparing spectroscopic estimates of NSCs and their hosts for a smaller sample of galaxies 
in the right panel of Fig. 9. This trend with galaxy mass is not as evident in the photometric measurements alone, but we regard the spectroscopic results as more reliable, given the near independence of the Lick index-based metallicities on age (e.g., Schiavon 2007).

M 54-a unique example: The only NSC for which we can obtain optical spectroscopy of individual member stars is the nucleus of the Sgr dwarf spheroidal galaxy (a.k.a. the globular cluster M 54). This makes it a key object for our understanding of NSCs, especially for low-mass early-type galaxies $\left(M_{\star} \sim 3 \times 10^{8} M_{\odot}\right)$. The dominant population in this NSC is old and metal-poor $([\mathrm{Fe} / \mathrm{H}]=-1.5)$, with a small number of much younger $(\lesssim 2 \mathrm{Gyr})$ and more metalrich $([\mathrm{Fe} / \mathrm{H}]=-0.5)$ stars (Monaco et al. 2005; Siegel et al. 2007; Mucciarelli et al. 2017; Alfaro-Cuello et al. 2019, 2020). The old metal-poor population has light-element abundance variations similar to those found in globular clusters (Mucciarelli et al. 2017; Sills et al. 2019), and recent work by Alfaro-Cuello et al. (2019) suggests that it also has a significant spread in both age and metallicity. We include the dominant metal-poor component of M 54 in the left-hand panel of Fig. 9, and see that it fits with the general trend of low metallicities in similar mass galaxies.

\subsection{Kinematics of NSCs}

To measure the kinematic structure of NSCs, one needs to resolve their morphology into many resolution elements. The sensitivity and high spatial resolution required to do this only became available with the advent of adaptive optics facilities on ground-based 8-m class telescopes. Earlier studies without the aid of adaptive optics were only able to get integrated velocity dispersion measurements (e.g., Böker et al. 1999; Walcher et al. 2005; Barth et al. 2009).

As discussed in Sect. 2.2, coupling the advantages of adaptive optics with integral-field spectrographs enabled spatially resolved studies of NSCs in a number of nearby galaxies (Seth et al. 2008b, 2010). These studies showed that most NSCs rotate in the same sense as the underlying host galaxy. This is true for both spiral and spheroidal galaxies, although in spheroidals, the amount of NSC rotation is typically lower and their kinematics can be quite complex: some show little or no rotation (e.g., in NGC 205; Nguyen et al. 2018), while others have high angular momentum (e.g., FCC 47 Fahrion et al. 2019), or even counter-rotating stars (Seth et al. 2010; Lyubenova et al. 2013). The ratio $v / \sigma$ of rotational velocity $v$ and velocity dispersion $\sigma$ provides a sense of whether the NSC dynamics are dominated by rotation or random motion. Typical values of $(v / \sigma)_{\mathrm{r}_{\text {eff }}}$ (measured at the NSC effective radius) for NSCs in early-type galaxies are in the range of $0-0.5$ (Seth et al. 2010; Nguyen et al. 2018; Lyubenova and Tsatsi 2019). In comparison, the NSC of the Milky Way has $(v / \sigma)_{\mathrm{r}_{\text {eff }}}=0.6$ (Feldmeier et al. 2014). Another edge-on spiral galaxy, NGC 4244, has an even higher value of $(v / \sigma)_{\mathrm{r}_{\mathrm{eff}}} \sim 1$ (Seth et al. 2008a). A compilation of available kinematic data is shown in Fig. 11, which compares these rough NSC measurements with globular cluster measurements from Bianchini et al. (2013) and Kamann et al. (2018). While many NSCs fall in the 
same region of the diagram occupied by globular clusters, some are significantly more flattened and rapidly rotating. We caution that detailed modeling is required to infer the orbital structure of NSCs; for instance, three-integral models of the flattened and rapidly rotating NSC in NGC 4244 suggest that the object has a mildly negative vertical anisotropy (De Lorenzi et al. 2013), while models of the Milky Way NSC suggest tangential anisotropy at radii $<2 \mathrm{pc}$, with nearly isotropic orbits at larger radii (Feldmeier-Krause et al. 2017b).

We conclude that while some NSCs (mostly in spiral galaxies) can have significant levels of rotation, in general, they are pressure-supported stellar systems.

\subsection{Properties of the Milky Way nuclear star cluster}

The NSC at the heart of the Milky Way warrants a special attention, because its proximity ( $d=8.1 \pm 0.1 \mathrm{kpc}$, Gravity Collaboration et al. 2019; Do et al. 2019) offers a unique opportunity to study physical processes on scales that are impossible to resolve in external NSCs (at $d=8.1 \mathrm{kpc}, 1^{\prime \prime}=0.04 \mathrm{pc}$ ). In this section, we, therefore, provide a brief summary of the properties of the Milky Way NSC. For a more comprehensive overview, we refer the reader to the recent reviews by Genzel et al. (2010) and Schödel et al. (2014b).

- Morphology, luminosity, and mass: The Milky Way NSC was first detected by Becklin and Neugebauer (1968) and later put into context of the larger Galactic structure by Launhardt et al. (2002). Becklin and Neugebauer (1968) measured the FWHM of the Milky Way NSC to be $\sim 180^{\prime \prime}$ in K-band (i.e., 7.2 pc for $d=8.1 \mathrm{kpc}$ ), with a shape elongated along the Galactic plane. Based on widefield observations with the IRAS and COBE satellites, Launhardt et al. (2002) later described the Milky Way NSC as spherically symmetric and embedded in a larger disk structure, i.e., a nuclear stellar disk with a scale length of about $120 \mathrm{pc}$. However, due to the limited spatial resolution of their data, they did not further characterise the NSC itself. More recently, Schödel et al. (2014a) produced mid-infrared images of the Milky Way NSC from multi-band Spitzer data that were largely corrected for extinction. Contrary to the assumption of Launhardt et al. (2002), the Milky Way NSC appears to be intrinsically elliptical and flattened along the Galactic plane, with an ellipticity of $\epsilon=0.29 \pm 0.02$ (i.e., an axis ratio $q=b / a=0.71 \pm 0.02$; Schödel et al. 2014a).

Published values for the effective radius of the Milky Way NSC fall within the range of $r_{\text {eff }} \sim 110^{\prime \prime} \pm 11^{\prime \prime}$ (Spitzer $4.5 \mu \mathrm{m}$ ) to $178^{\prime \prime} \pm 51^{\prime \prime}$ (K-band), i.e., between $4.2 \pm 0.4 \mathrm{pc}$ and $7.2 \pm 2.0 \mathrm{pc}$ (Schödel et al. 2014a; Fritz et al. 2016). The total luminosity of the Milky Way NSC is $L_{4.5 \mu m}=4.1 \pm 0.4 \times 10^{7} L_{\odot}$ Schödel et al. (2014a). At NIR wavelengths, Fritz et al. (2016) obtain $\mathrm{M}_{\mathrm{Ks}}=-16.0 \pm 0.5$ within the effective radius, which corresponds to a total $\mathrm{L}_{\mathrm{Ks}}=5.2 \pm 3.0 \times 10^{7} L_{\odot}$, consistent with the earlier measurement of Launhardt et al. (2002).

A number of recent studies have derived the total stellar mass of the Milky Way NSC. Using both photometric and dynamical methods, the results fall within the range between $\sim 2.1 \pm 0.7 \times 10^{7} M_{\odot}$ and $4.2 \pm 1.1 \times 10^{7} M_{\odot}$ (Schödel et al. 
2014a; Feldmeier et al. 2014; Chatzopoulos et al. 2015; Fritz et al. 2016; Feldmeier-Krause et al. 2017b). Remarkably, this range is entirely consistent with the earliest mass estimates of the Milky Way NSC (Becklin and Neugebauer 1968; Launhardt et al. 2002).

- Stellar populations and star formation history: The majority of the resolved stars in the Milky Way NSC are old ( $>5$ Gyr) and evolved (spectral types K and M) giant and supergiant stars, as well as helium-burning stars on the horizontal branch and the 'red clump', which have temperatures of $\sim 3000$ to $5000 \mathrm{~K}$, and low-to-intermediate masses $\left(M_{\star} \sim 0.5\right.$ to $4 M_{\odot}$, Blum et al. 2003; Genzel et al. 2010; Feldmeier-Krause et al. 2017a). This is consistent with $\sim 80 \%$ of the stellar mass having formed more than $5 \mathrm{Gyr}$ ago, with an initially high star formation rate 10 Gyr ago that dropped to a minimum about 1-2 Gyr ago, and then increased again over the past few 100 million years (Blum et al. 2003; Pfuhl et al. 2011; Nogueras-Lara et al. 2019a).

In addition to the old stars, there is a population of about 200 massive and young Wolf-Rayet and O- and B-type stars that are confined to the central $0.5 \mathrm{pc}$ (Allen et al. 1990; Krabbe et al. 1991; Ghez et al. 2003; Bartko et al. 2010; Pfuhl et al. 2011; Feldmeier-Krause et al. 2015). The ages of these young stars are very well constrained within the range 3-8 Myr (Paumard et al. 2006; Lu et al. 2013). The total mass in young stars is between 14,000 and 37,000 $M_{\odot}$ (measured via the K-band luminosity function, Lu et al. 2013); the measurement of photometric masses of the O/B stars gives a consistent lower limit $\geq 12,000 M_{\odot}($ Feldmeier-Krause et al. 2015). These stars have very likely formed in situ, because the time which it would take to bring them to the center is in conflict with their very young age. The lack of young stars outside of the central $0.5 \mathrm{pc}$ is further evidence against the infall of young star clusters (e.g., Feldmeier-Krause et al. 2015). In summary, the stars in the NSC are on average younger and more metal-rich than the stars in the Galactic bulge (Ness et al. 2013; Feldmeier-Krause et al. 2015, 2017a; Nogueras-Lara et al. 2018; Schultheis et al. 2019).

- Mass density: The average surface mass density of the Milky Way NSC is $\sim 2 \times 10^{5} M_{\odot} / \mathrm{pc}^{2}$ within the effective radius, and $\sim 2.5 \times 10^{6} M_{\odot} / \mathrm{pc}^{2}$ within the central $\sim 0.5$ pc (Genzel et al. 2010; Feldmeier et al. 2014; Schödel et al. 2014a, b). Schödel et al. (2018) derived the three-dimensional stellar mass density for the Milky Way NSC on scales of $0.01 \mathrm{pc}$ to $2.6 \pm 0.3 \times 10^{7} M_{\odot} / \mathrm{pc}^{3}$.

- Kinematic structure: A variety of observational techniques have revealed that the Milky Way NSC is rotating in the same direction as the overall Galactic disk (McGinn et al. 1989; Lindqvist et al. 1992; Genzel et al. 1996; Feldmeier et al. 2014). The line-of-sight velocity of the stars at the NSC effective radius is about $50 \pm 3 \mathrm{~km} / \mathrm{s}$, while the stellar velocity dispersion at the same radius is slightly higher (McGinn et al. 1989; Feldmeier et al. 2014, $60 \pm 5 \mathrm{~km} / \mathrm{s}$ ). The resulting value of $v / \sigma_{\mathrm{r}_{\text {eff }}} \sim 0.8$ at the effective radius actually represents a maximum, with values between 0.2 and 0.8 for smaller radii.

Using a long-slit drift scanning technique, Feldmeier et al. (2014) constructed contiguous velocity and velocity dispersion maps of the central $8 \mathrm{pc} \times 4 \mathrm{pc}$. 
These maps show that the major axis of rotation is offset with respect to the Galactic plane by about $9^{\circ}$ (see their Fig. 8). This finding was confirmed by Fritz et al. (2016). In addition, the line-of-sight velocity map shows a perpendicular rotating substructure, which may be the result of star cluster infall (Feldmeier et al. 2014; Tsatsi et al. 2017).

- Supermassive black hole: At the center of the Milky Way, NSC resides a supermassive black hole, SgrA*, with a mass of $4.04 \pm 0.06 \times 10^{6} M_{\odot}$ (Gravity Collaboration et al. 2018; Do et al. 2019). Given the NSC mass of $\sim 3 \times 10^{7}$ $M_{\odot}$, this implies that in the case of the Milky Way, the mass fraction of the black hole relative to the nuclear star cluster is $\sim 13 \%$.

In summary, the Milky Way appears to contain a rather typical example for an NSC, because many of its properties are entirely consistent with the general sample of NSCs in external galaxies (see also Table 2).

\subsection{Properties of the $\mathbf{M} 31$ nuclear star cluster}

Besides the Milky Way NSC, the nucleus of M 31 also deserves a special attention, because it is one of the nearest extragalactic NSCs (distance $d=785 \mathrm{pc}$; McConnachie et al. 2005), and in fact, it was the first NSC to be discovered, as described in Sect. 2. As will become evident, its properties are quite different from those of the Milky Way NSC. For example, it represents a rare example of a nucleus in which the central black hole outweighs the NSC.

HST observations have made it clear that the M 31 NSC has a complicated morphology (Lauer et al. 1993; King et al. 1995; Lauer et al. 1998). The surface brightness of the NSC rises above the M 31 bulge at a radius of $\sim 5 "(\sim 20 \mathrm{pc})$ (Kormendy and Bender 1999; Peng 2002). The outermost component of the NSC is nearly spherical $(q=0.97)$, with an effective radius of $r_{\mathrm{eff}}=3.2 "(\sim 12 \mathrm{pc})$ and a mass of $2.8 \times 10^{7} M_{\odot}$ (Peng 2002). Embedded within this are two sources (dubbed P1 and P2) which are both offset from the central black hole and thought to be part of a flattened eccentric disk (discussed in more detail below). This disk shows strong rotation (with an amplitude up to $\sim 250 \mathrm{~km} / \mathrm{s}$ Lockhart et al. 2018), and has an effective radius of $<1$ " $(<4 \mathrm{pc})$, and a total mass of $2.1 \times 10^{7} M_{\odot}$, resulting in a total NSC mass of $\sim 5 \times 10^{7} M_{\odot}$ (Peng 2002). Spectroscopic fitting shows that the stars in the central $\sim 5 "$ are old (7-13 Gyr) and extremely metal-rich $([\mathrm{Z} / \mathrm{H}]=0.3-0.5)$, i.e., even more metal-rich than the stars in the surrounding bulge (Saglia et al. 2010). The three-dimensional stellar mass density of the NSC on scales of $0.1 \mathrm{pc}$ is $\sim 2 \times 10^{6} M_{\odot} / \mathrm{pc}^{3}$ (Lauer et al. 1998).

The NSC of M 31 hosts an SMBH of $\sim 1.1-2.3 \times 10^{8} M_{\odot}$ (Peiris and Tremaine 2003; Bender et al. 2005). Immediately surrounding the central black hole is a UV-bright source with an A-type spectrum known as P3 (Bender et al. 2005). This source is blue, not because of AGN light but rather, because it is dominated by hot stars. The spectrum and high-resolution images are well described by a $10^{4} M_{\odot}$ 100-200 Myr old stellar population (Bender et al. 2005; Lauer et al. 2012). These stars are rotating in a co-planar way with the older eccentric disk (Bender 
et al. 2005). This young population also appears to be associated with a lack of NIR $K$-band emission (Lockhart et al. 2018).

The morphological structure of the central region of M 31's NSC is best explained by an eccentric disk model, composed of stars traveling on nearly Keplerian orbits around a black hole (Tremaine 1995). This model reproduces most of the features seen in HST photometry, in particular the bright off-center source P1 which is the apocenter region of the disk. Recent integral-field kinematics are also well-fit by this model (Lockhart et al. 2018), and those data suggest a slow precession rate for the disk. The eccentric disk model also explains the fact that the velocity dispersion peak is offset by $\sim 0.2^{\prime \prime}$ from the UV peak, assumed to mark the location of the supermassive black hole (Bacon et al. 2001). Moreover, the eccentric stellar disk model can explain the formation of young stars at the center of M 31's NSC (Chang et al. 2007). The disk creates a non-axisymmetric perturbation to the potential and drives gas into the inner parsec around the SMBH. Chang et al. (2007) show that stellar mass loss from P1 and P2 would be sufficient to create a gravitationally unstable gaseous disk of $\sim 10^{5} M_{\odot}$ every $0.1-1 \mathrm{Gyr}$, consistent with the young age of P3 ( 200 Myr).

Although the mass of the MW and M 31 NSCs is quite comparable, the ratio of this mass to the black hole mass is dramatically different, with the $\mathrm{M} 31 \mathrm{BH}$ being $\sim 3 \times$ the mass of the NSC, while in the Milky Way, it makes up only $<15 \%$. The dominance of the BH mass accounts for M 31's eccentric disk structure (Tremaine 2019), providing us with a useful nearby case study in this regime which is more typical for higher mass galaxies (see Sect. 8).

\section{Nuclear star clusters and their host galaxies}

In this section, we consider how NSCs are related to the host galaxies which they live in. We first discuss the overall scaling of NSC mass with galaxy properties, and then investigate how other NSC properties vary with galaxy mass and type.

\subsection{Scaling relations of NSCs}

The most straightforward quantities to compare between galaxies and NSCs are their luminosities and stellar masses, and there is a substantial body of literature that has examined these correlations. The NSC luminosity was first compared to the host galaxy/bulge luminosity for small samples of galaxies by Balcells et al. (2003) and Graham and Guzmán (2003). Inspired by this, as well as the prominent black hole scaling relations, subsequent papers examined NSC-galaxy scaling relations using both luminosity and mass (Ferrarese et al. 2006a; Wehner and Harris 2006; Rossa et al. 2006; Balcells et al. 2007; Seth et al. 2008b; Erwin and Gadotti 2012; Scott and Graham 2013; den Brok et al. 2014; Capuzzo-Dolcetta and Tosta e Melo 2017; Sánchez-Janssen et al. 2019a).

While it was at one point claimed that the NSC and BH scaling relations may be comparable (Ferrarese et al. 2006a; Wehner and Harris 2006), evidence is mounting 
that the NSC scaling relations are distinct from the $\mathrm{BH}$ scaling relations (Leigh et al. 2012; Erwin and Gadotti 2012; Scott and Graham 2013).

In the left panel of Fig. 12, we show a compilation of NSC and galaxy masses. The NSC sample is the same as in Fig. 3, with the addition of NSCs with masses measured using stellar population model fits or dynamical estimates (plotted as stars; Erwin and Gadotti 2012; Nguyen et al. 2018). The best-fit log-linear relation to the full sample of 407 NSC and host galaxy mass estimates is:

$$
\log M_{\mathrm{NSC}}=0.48 \log \left(\frac{M_{\star}}{10^{9} \mathrm{M}_{\odot}}\right)+6.51 .
$$

Bootstrapping errors on both parameters of this fit are $\sim 0.04$ dex, while the scatter around the fit is $\sim 0.6$ dex. While the errors on estimated $\mathrm{M} / \mathrm{L}$ ratios for the individual measurements are large and heterogeneous, they should be below $\sim 0.3$ dex (Roediger and Courteau 2015), which implies that the scatter is primarily intrinsic.

This NSC-stellar mass-scaling relation is consistent with the previous results (Balcells et al. 2003; Scott and Graham 2013; den Brok et al. 2014; SánchezJanssen et al. 2019a, but also see Georgiev et al. 2016 who find a steeper relation), and suggests that roughly $M_{\mathrm{NSC}} \propto M_{\star}^{1 / 2}$. This sub-linear trend implies that NSCs in lower mass galaxies contain a higher fraction of the galaxy mass than in higher mass galaxies. At $10^{9} M_{\odot}$, this mass is about $0.3 \%$ of the total galaxy mass, in agreement with the findings from Côté et al. (2006). The NSC-galaxy mass fraction is shown in the right panel of Fig. 12, which shows clearly that the mass scaling is very similar for both early- and late-type galaxies. We will discuss this result in the context of formation models in Sect. 7.

There are hints in both panels that the high mass end of NSCs may behave somewhat differently, with a possible steepening of the NSC mass-scaling slope. For early types, this steepening has previously been noted by den Brok et al. (2014) and by Sánchez-Janssen et al. (2019a). At the same time, Scott and Graham (2013) exclude these more massive objects as apparent nuclear stellar disks with larger effective radii rather than NSCs. Despite the fact that, given our inclusive definition of NSCs, these objects remain in our sample, we nonetheless maintain a shallow slope even at higher masses when including all objects in the fit. On the other hand, if we restrict ourselves to the smaller subsample of objects with more accurate NSC mass measurements (plotted as stars), we do indeed find a steeper slope, as also found by Georgiev et al. (2016). More specifically, fitting just these galaxies which primarily contain massive NSCs in late-type galaxies, we get a nearly linear relationship:

$$
\log M_{\mathrm{NSC}}=0.92 \log \left(\frac{M_{\star}}{10^{9} \mathrm{M}_{\odot}}\right)+6.13
$$

with bootstrapping uncertainties of $\sim 0.18$ dex on both quantities. This line is shown as a dot-dashed line in the left panel of Fig. 12. Hints of this linear massscaling relationship are also seen in the full sample of galaxies in the right panel, where the early-type galaxies have a bump above the best-fit relation above $10^{9} M_{\odot}$, 
while the late-type galaxies show a modest flattening at the highest mass end. We note that the lower mass fraction in the highest mass early-type bin in the right panel is due to our inclusion of galaxies from the Lauer et al. (2005) sample, which have not been included in previous scaling relation work (i.e., Scott and Graham 2013; Sánchez-Janssen et al. 2019a). Surprisingly, these objects seem to follow the trend found for low-mass early-types, rather than continue the upturn found by SánchezJanssen et al. (2019a).

\subsection{Other correlations between NSCs and their hosts}

Throughout this review, we have already touched on several links between NSCs and their hosts (apart from their mass-scaling relations discussed above) which we now summarize. In Sect. 4, we showed that there is a clear dependence of the nucleation fraction on galaxy stellar mass. At low masses, this dependence seems to be independent of galaxy type, rising steadily up to $\sim 10^{9} M_{\odot}$ where the occupation fraction peaks. Moreover, the mass-scaling relation discussed in the previous subsection also seems to depend on galaxy stellar mass but not on galaxy type.

The stellar populations of NSCs (Sect. 5.4) also are linked to their host galaxy masses, at least in early-type galaxies. Specifically, at low masses, NSC metallicities have a wide range and are often lower than those of their hosts (Fig. 9), while at high masses, they are always higher. A comparison of the integrated colors of earlytype NSCs vs. their hosts shows a trend consistent with this change in metallicity: Turner et al. (2012) and Sánchez-Janssen et al. (2019a) find that their lowest mass galaxies have NSCs that are typically bluer than their hosts (suggesting that the NSCs are more metal-poor than their hosts), while many higher mass galaxies have NSCs that are redder (consistent with higher NSC metallicities). The metallicities of older stars in late-type galaxies are very difficult to constrain (Kacharov et al. 2018), and existing late-type spectroscopic measurements are all for galaxies with $M_{\star}>10^{9} M_{\odot}$. Therefore, it remains unknown if a similar trend towards lowmetallicity NSCs exists in late-type galaxies, although the colors of NSCs in these galaxies do seem to be typical of metal-poor GCs (Georgiev et al. 2009).

While the occupation fraction and mass scaling of NSCs in early-type and latetype galaxies are remarkably similar, there are some NSC properties that seem to differ between the two galaxy types. Most obviously, spectroscopic studies suggest that the ages and metallicities of stars in NSCs are correlated with those of their host galaxies (Rossa et al. 2006; Kacharov et al. 2018), and young $<100 \mathrm{Myr}$ populations are ubiquitous only in late-type spirals (Walcher et al. 2006). The size-mass relation also appears to differ, with NSCs in early-type galaxies being larger at a given NSC mass (and galaxy stellar mass) than late-type NSCs (Georgiev et al. 2016). This observation may be related to the young stars in these NSCs being centrally concentrated (Carson et al. 2015). Another possible consequence of the presence of young stars is the large ellipticity of some young clusters (Fig. 5), which may be due to disky distributions of young stars (Seth et al. 2006; Carson et al. 2015). 
We will return to these observations to help constrain formation models of NSCs in the next section.

\section{Scenarios for the formation and growth of nuclear star clusters}

In this section, we will attempt to address the questions raised in Sect. 1, namely when and how NSCs form, and whether the answer depends on the properties of their host galaxy. We emphasize that the mechanisms for the initial NSC formation and their subsequent growth do not necessarily have to be the same.

\subsection{Initial formation of NSCs}

A high-level question which we would like to address first is whether NSCs initially form before, together with, or after their hosts. Since they lie deepest in the potential well of galaxies where gas densities are likely to be highest, seed NSCs could be among the earliest structures to form in a galaxy. In this scenario, one would expect that at least some NSC stars are both older and more metal-poor than the stars in the surrounding galaxy.

In principle, it is easier to observationally constrain the metallicity of individual stars, rather than their age. Individual metal-poor stars have been identified at the center of the two nearest NSCs: the Sgr dSph NSC (M 54) contains a dominant old metal-poor population (Mucciarelli et al. 2017; Alfaro-Cuello et al. 2019), while in the Milky Way NSC, a small number of stars with $[\mathrm{Fe} / \mathrm{H}]<-1$ are found (Do et al. 2015; Ryde et al. 2016; Rich et al. 2017). In fact, the Milky Way is the only case where we can ascertain that such a population exists alongside a dominant population of younger and more metal-rich stars.

In the general case of more distant NSCs, however, one has to rely on integrated light measurements, which makes it nearly impossible to constrain the metal-poor population (Seth et al. 2010; Kacharov et al. 2018). The exception is when the metal-poor population dominates the light of the NSC, as it does in some low-mass early-type galaxies (see Fig. 9 and Sect. 5.4). The fact that these NSCs are dominated by stars that are more metal poor than those of the stellar body of their host galaxy implies that NSC stars must have either formed in situ before the stars that surround them, or they formed elsewhere from less enriched gas, and then inspiraled to the nucleus (see next subsection for details). Such early formation of dense clusters in dark matter halos has a rich theoretical history, focused primarily on the goal of forming globular cluster-like objects (see recent review by Renaud 2018). One particularly interesting scenario that may be relevant for NSCs is the proposal by Cen (2001) that during reionization, shocks could compress gas in the inner regions of dark matter halos, thus forming clusters with masses as high as $10^{6} M_{\odot}$.

Ongoing efforts to model globular cluster formation in cosmological simulations (e.g., Li and Gnedin 2019; Ma et al. 2019; Lahén et al. 2019) have the potential to address the important question of how quickly galaxies acquire their NSCs, especially if they include the effects of dynamical friction. However, given the 
current lack of strong observational constraints and theoretical work on the initial formation of NSCs, we now turn our focus to understanding their subsequent growth.

\subsection{Globular cluster infall and merging}

Soon after the breakthrough observation of the M 31 NSC by Light et al. (1974), the first formation scenario for NSCs was offered by Tremaine et al. (1975). They suggested that the compact nuclear structures in M 31 and similar galaxies will inevitably be formed when globular clusters are 'dragged' into the nucleus by dynamical friction created as they orbit through the stellar body of their host galaxy. The strength of this dynamical friction depends linearly on the mass of the cluster, and thus, the most massive clusters are the most likely to inspiral into and form the NSC. The strength of the dynamical friction also depends inversely on the velocity $\left(\propto 1 / v^{2}\right)$ of the stars in the host galaxy. This scenario has received much attention over the years, both observationally and theoretically.

On the observational side, it has been pointed out that the radial profiles of GC systems in early-type galaxies seem to indicate a deficit of massive GCs in the inner parts of many galaxies (Lotz et al. 2001; Capuzzo-Dolcetta and MastrobuonoBattisti 2009), potentially indicating that dynamical friction has driven them into the nucleus. Additional evidence comes from the recent observation that in low-mass early-types, the nucleation fraction closely tracks the fraction of galaxies that host (non-nuclear) globular clusters (Sánchez-Janssen et al. 2019a). In addition, the previous work has shown that the specific frequency of globular clusters (the number of globular clusters per unit galaxy luminosity) is substantially higher in nucleated early-type dwarfs than in their non-nucleated counterparts (Miller and Lotz 2007; Lim et al. 2018).

An additional interesting finding is that in early-type galaxies, the total luminosity/mass in globular clusters and in NSCs seems to be nearly equal. This was first noted by Côté et al. (2006) who found that the typical NSC-to-galaxy luminosity ratio in their sample of Virgo early-type galaxies $(0.3 \%)$ was nearly identical to the GC system-to-galaxy mass ratio calculated by McLaughlin (1999). Taking this a step further, den Brok et al. (2014) showed that the typical GC system luminosity derived from previous work is within a factor of $\sim 2$ of the NSC luminosity for their large sample of Coma galaxies with masses between roughly $10^{7}$ and $10^{9} M_{\odot}$. The persistence of this mass fraction similarity between GCs and NSCs over a wide range of galaxy masses is something existing infall models which have yet to explain.

The presence of metal-poor stars in low-mass early-type galaxy nuclei discussed in Sect. 5.4, Fig. 9, and, in the previous subsection, may also support the dynamical friction scenario, as it provides a natural way to bring more metal-poor stellar populations to the center of the galaxy. The massive star clusters that can inspiral to NSCs appear to be more efficiently produced at low metallicities (Pfeffer et al. 2018). Therefore, NSCs more metal-poor than their surrounding hosts are likely an expectation of dynamical friction-driven infall models. However, we caution that 
the surviving globular cluster population can be quite different from the population of clusters that formed and fell into the NSC (or were subsequently destroyed) (e.g., Lamers et al. 2017). We also note here that although we use the term globular clusters in this discussion, the infalling clusters can also be younger massive clusters (e.g., Agarwal and Milosavljević 2011).

A large number of simulations and semi-analytic models have quantified the efficiency of dynamical friction over a wide range of starting conditions for the GC systems and their host galaxies. The general picture that has emerged is the following. Provided that early star formation in the host galaxy produces a sufficient number of high-mass $\left(M_{\star} \gtrsim 10^{5} M_{\odot}\right)$ clusters, dynamical friction, indeed, provides a plausible mechanism to form an NSC on time scales much shorter than a Hubble time (e.g., Capuzzo-Dolcetta 1993; Oh and Lin 2000; Lotz et al. 2001; Agarwal and Milosavljević 2011; Neumayer et al. 2011).

Detailed N-body simulations of the dynamical friction-induced merger process in galactic nuclei, first performed by Capuzzo-Dolcetta and Miocchi (2008a, b), have shown consistency with some properties of observed NSCs. For instance, Hartmann et al. (2011) found they could match the density and shape of some local, wellresolved NSCs in late-type galaxies with merging star cluster simulations, but that matching the kinematics of the clusters likely requires roughly half of the NSC mass to stem from accreted gas and subsequent in situ star formation. In a similar vein, Antonini et al. (2012) successfully reproduced the density profile of the Milky Way NSC from the accretion of globular clusters, but also concluded that the observed luminosity function requires younger stars and, therefore, suggested that only about half of the NSC mass can originate from globular cluster inspirals. The rotation of NSCs was investigated by Tsatsi et al. (2017), who found that their models could match the observed flattening (Schödel et al. 2014a) and rotation signature (Feldmeier et al. 2014) of the Milky Way NSC (Figs. 10, 11).

Another class of simulations are semi-analytic models that follow the evolution of GC systems with dynamical friction to track the growth of their NSCs. These models are able to produce some of the properties of both the present-day NSCs and the 'left over' GC systems (Antonini 2013; Gnedin et al. 2014). Antonini et al. (2012) and Antonini (2013) find a size-luminosity (or mass) relation of $r_{\text {eff }} \propto M_{\mathrm{NSC}}^{0.5}$ for a small number of mergers, similar to the one derived by Côté et al. (2006) for early-type NSCs, but a little steeper than the size-mass relationship of $r_{\text {eff }} \propto M_{\mathrm{NSC}}^{0.35}$ found by Georgiev et al. (2016). Figure 7 shows the size-mass relations for NSCs, with the upper dashed line indicating the power-law $r_{\mathrm{eff}} \propto M_{\mathrm{NSC}}^{0.5}$. At the low-mass end, it is clear that the observations indicate a shallower relation than this, while at the high-mass end, the relation seems to be a good match to the data. The simulations of Gnedin et al. (2014) find that the fraction of galaxy mass contained within the NSC scales as $M_{\mathrm{NSC}} / M_{\star} \propto M_{\star}^{-0.5}$ or, alternatively, $M_{\mathrm{NSC}} \propto M_{\star}^{0.5}$. In Sect. 6.1 and Fig. 12, we find a very good match to this mass-scaling prediction for the full sample of both early- and late-type galaxies. We note, however, that while the power-law slope matches, the normalization which we find is about an order of magnitude lower than the $0.25 \%$ of mass suggested by Gnedin et al. (2014) for galaxies with $M_{\star}=10^{11} M_{\odot}$. 

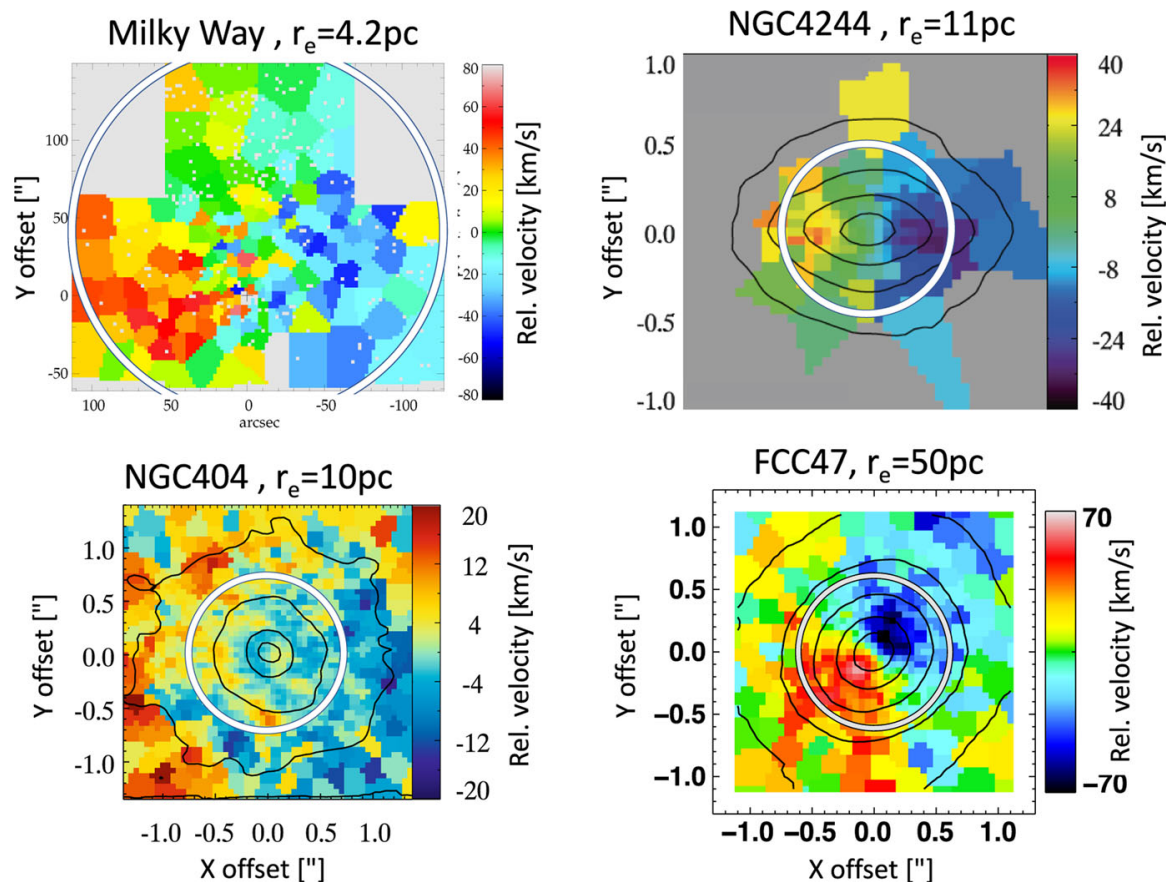

Fig. 10 Examples for rotating NSCs: top: spiral galaxies Milky Way (left, Feldmeier et al. 2014) and NGC 4244 (right, Seth et al. 2008b). Bottom: early-type galaxies NGC 404 (left, Seth et al. 2010) and FCC 47 (right, Lyubenova and Tsatsi 2019). In all panels, the effective radius of the NSC is marked with a white circle

One additional interesting body of work on dynamical friction-induced cluster inspiral relates to the Fornax dSph galaxy, which has five bright globular clusters, but is not nucleated. The galaxy is dark matter dominated, and Goerdt et al. (2006) find that if the galaxy possessed an NFW halo (with a central dark matter cusp), the globular clusters should sink to the center over a timescale between 1.5 and 5 Gyr. They interpret this as evidence for a flattened (cored) dark matter halo, which would lengthen the timescale for cluster inspiral. This issue remains an area of active research (e.g., Arca-Sedda and Capuzzo-Dolcetta 2017; Boldrini et al. 2019; Meadows et al. 2019), and highlights the possibility that the properties of NSCs and star cluster populations in the lowest mass galaxies may be able to contribute to our understanding of dark matter halos. This is especially true if these studies can be expanded from a single galaxy to large samples of low-mass NSCs and GCs that span a wide range in galaxy mass.

To summarize, the infall of massive stellar clusters into the galaxy nucleus is an expected (and perhaps unavoidable) process that has likely contributed a significant amount of stellar mass to present-day NSCs. However, the cluster infall scenario alone cannot easily explain the presence of young stars in many NSCs (see Sect. 5.4), which is why additional mechanisms are needed to fully describe NSC evolution. 


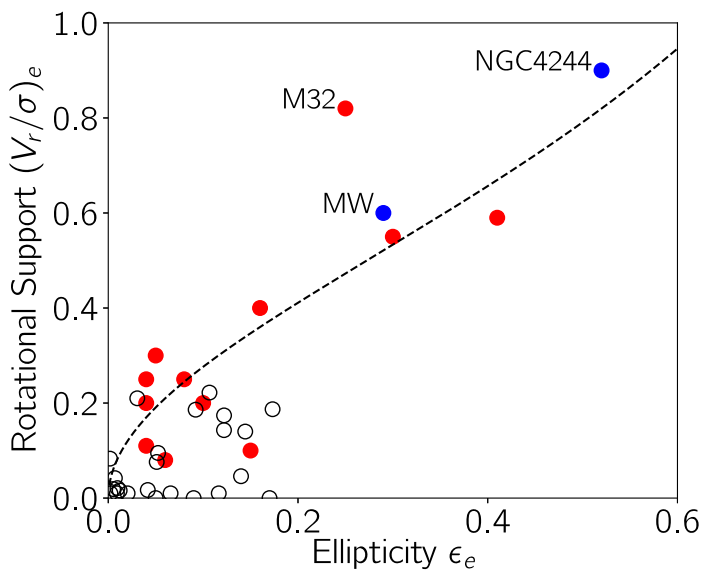

Fig. 11 Many NSCs are rapidly rotating. This plot shows the strength of rotation $(v / \sigma)_{\mathrm{r}_{\mathrm{eff}}}$ vs. the ellipticity $\epsilon$ both estimated at the half-light radius for NSCs (colored by Hubble type of their host galaxy; from Seth et al. 2008b, 2010; Feldmeier et al. 2014; Nguyen et al. 2018; Lyubenova and Tsatsi 2019) and GCs (open circles from Bianchini et al. 2013; Kamann et al. 2018). The dashed line shows the expected trend for an edge-on isotropic, oblate rotator (Cappellari 2016); many of the more rapidly rotating NSCs fall close to this trend. Both the Milky Way and NGC4244 are, indeed, seen edge-on; lower inclinations move objects to the left of the line (and then down). Note that the plotted NSCs sit at the center of the potential well of a galaxy, and should not necessarily be compared to entire galaxies
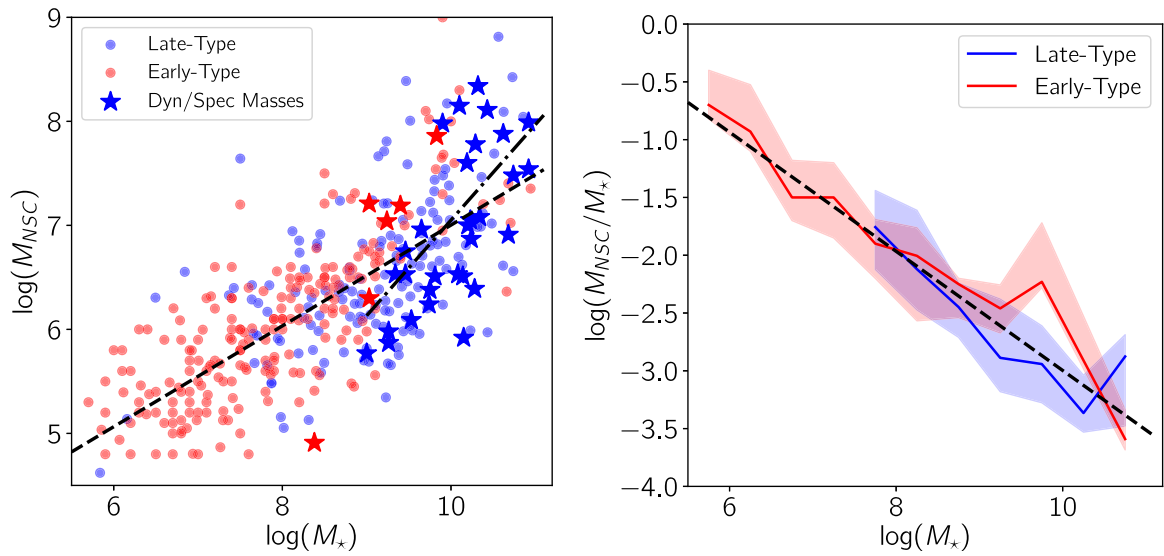

Fig. 12 The masses of nuclear star clusters correlate with galaxy masses, but higher mass galaxies have a lower fraction of their mass in their NSC. Left: Galaxy and NSC masses for galaxies in which both quantities are available. The compilation of dynamical and spectroscopically modeled NSC masses from Erwin and Gadotti (2012) is shown with stars, while all other masses are derived from colors using stellar population models with a Chabrier or Kroupa IMF (Georgiev et al. 2016; Spengler et al. 2017; OrdenesBriceño et al. 2018; Sánchez-Janssen et al. 2019a). Galaxies have been divided by their Hubble types into early and late types. Right: the mass fraction of galaxies in NSCs as a function of galaxy mass, using the data from the left panel. The line indicates the median galaxy within each mass bin, while shaded regions show the 25th and 75th percentiles of the distribution 


\subsection{In situ star formation}

To explain the presence of young stellar populations in many NSCs in late-type hosts, in situ star formation is usually invoked, which occurs when gas reaches the central few pc and triggers an intense burst of star formation. This mechanism is potentially repetitive, driven by the mechanical feedback from supernovae and strong stellar winds which 'blows out' the gas and temporarily halts star formation until the turbulent energy has been dissipated, and the gas re-assembles in the nucleus on timescales of $\sim 10^{8}$ years (Loose et al. 1982). One complication in separating this scenario from the cluster inspiral scenario is that young stars can also inspiral to the NSC if they form sufficiently close to the center (e.g., Gerhard 2001; Agarwal and Milosavljević 2011; Neumayer et al. 2011).

In this section, we re-analyze the evidence for young stellar populations in NSCs from Sect. 5.4, with a focus on arguments for in situ star formation. The Milky Way NSC has a population of very young stars with ages between 3 and $8 \mathrm{Myr}$ at radii $<$ $0.5 \mathrm{pc}$ (see Sect. 5.6). The presence of such young stellar ages argues in favor of in situ formation rather than the inspiral of a young cluster, because the latter should leave detectable remnants at larger radii which are not found (Paumard et al. 2006; Lu et al. 2009; Feldmeier-Krause et al. 2015). A similar concentration of young stars is seen in many other nearby NSCs including M 31 (Bender et al. 2005; Georgiev and Böker 2014; Carson et al. 2015), as well as in some of the closest early-type galaxies (Nguyen et al. 2017, 2019). These younger populations are often flattened and rotating, as expected if they formed from gas accreted into the nucleus (Seth et al. 2006, 2008b; Carson et al. 2015; Nguyen et al. 2019, see also Sect. 5.5]). Integrated spectroscopy of NSCs in $>10^{9} M_{\odot}$ late-type spirals shows that they all contain stellar populations younger than $10^{8}$ years (Walcher et al. 2006; Seth et al. 2006; Kacharov et al. 2018). The mass of these young populations is of order $10^{5} M_{\odot}$, sufficient to explain the total mass of the NSC if they had formed through similar formation episodes repeated every $\sim 100$ Myr over a Hubble time (Walcher et al. 2006; Seth et al. 2006). The presence of emission lines also indicates the presence of very young stars $(<10 \mathrm{Myr})$ in some NSCs (Walcher et al. 2006; Seth et al. 2008a). Overall, there is strong evidence that in situ star formation occurs frequently in NSCs, and is likely the dominant growth mechanism for NSCs in late-type galaxies.

The starbursting blue compact dwarf Henize $2-10$ is a special case, which we mention to demonstrate that young cluster accretion can occur in some cases. In this galaxy, no NSC is currently visible, despite evidence for a central BH (Reines et al. 2011, 2016, although see Hebbar et al. 2019). However, there are several young and massive star clusters within the central $\sim 100 \mathrm{pc}$ that, due to dynamical friction, will likely inspiral and merge to form an NSC on timescales of a few hundred Myr (Nguyen et al. 2014; Arca-Sedda et al. 2015). However, based on the evidence presented above, this case appears to be somewhat atypical, with truly centralized star formation being the more common mode of building NSCs. This triggers the question of how to transport the necessary amounts of gas from larger radii to the central few pc. 
It has long been accepted that interactions between gas-rich galaxies, a.k.a. 'wet mergers', will inevitably lead to a central gas concentration in the merger remnant (Mihos and Hernquist 1994). The ensuing localized star formation will leave behind "a sharp break in the surface density profile at a few percent of the effective radius" which matches well the extent of NSCs in early-type hosts (Côté et al. 2007). More recent work by Hopkins and Quataert (2010a, b) simulates mergers at high resolution to determine the amount of star formation in the nucleus (and the black hole accretion), and finds that they can form objects similar to the asymmetric disk in M 31. However, the fact that NSCs are also found in most 'pure' disk galaxies which have never experienced any substantive interactions argues that even without mergers, gas can reach the nuclear regions of galaxies. In fact, there are various secular mechanisms that cause gas to accumulate in the galaxy nucleus:

- bar-driven gas infall: gas responding to a non-axisymmetric potential (e.g., produced by a stellar bar) will move inwards, and will ultimately reach the central few pc (e.g., Shlosman et al. 1990). This can occur both via regular flows in the so-called 'nuclear spirals' (e.g., Maciejewski 2004; Kim and Elmegreen 2017) or through angular momentum loss in star formation rings caused by dynamical resonances (e.g., Hunt et al. 2008). One clear example of bar-driven gas infall is in the late-type spiral NGC 6946, where Schinnerer et al. (2006, 2007) find $1.6 \times 10^{7} M_{\odot}$ of molecular gas within a radius of $30 \mathrm{pc}$. Star formation in this gas will likely lead to the formation (or growth) of a massive NSC. This suggests that NSC formation may still be ongoing in the local universe (Emsellem et al. 2015).

- dissipative nucleation: a similar process occurring at high redshift that involves clumpy star formation in nuclear spirals, and the ensuing infall and merging of such clumps, has been proposed by Bekki et al. (2006) and Bekki (2007) to explain the nucleation of spheroidal galaxies that are now devoid of gas. They suggest that the low nucleation rate in the least massive galaxies (see Fig. 3) is a natural consequence of the fact that feedback from stellar winds and supernovae will more easily expel the gas before it reaches the nucleus.

- tidal compression: Emsellem and van de Ven (2008) show that for galaxies without a pre-existing NSC and a reasonably flat density profile (i.e., with Sérsic index $n \lesssim 3.5$ ), the tidal forces acting on the gas become compressive within about $0.1 \%$ of the galaxy's effective radius, which typically corresponds to a few pc. They point out that NSC formation via this mechanism would naturally explain the observed scaling between the mass of the NSC and that of the host spheroid.

- magneto-rotational instability: Milosavljević (2004) suggests that a differentially rotating disk of neutral gas subjected to a weak magnetic field will develop the so-called magneto-rotational instability, which causes radial gas transport towards the nucleus. Using conditions typical for those in late-type spiral galaxies such as M 33, they conclude that the expected gas inflow rates are sufficient to produce a $10^{6} M_{\odot}$ NSC within about a Gyr. 
Overall, it is clear that gas infall into the central few pc and the ensuing nuclear star formation are a natural and expected process, which almost certainly contributes to the growth of NSCs in gas-rich galaxies.

Numerically simulating in situ NSC star formation in a galactic or even cosmological context is challenging, although some initial work has been done (Hopkins and Quataert 2010a, b; Guillard et al. 2016; Brown et al. 2018). In a more specific context, Aharon and Perets (2015) have tried to simulate whether the Milky Way NSC can be built from successive generations of star formation, and show that they can potentially explain the varying density profiles of different stellar populations. Using a different approach, Guillard et al. (2016) perform galaxy-scale simulations to investigate a 'hybrid' wet-merger formation scenario, which combines cluster infall with in situ star formation in a gas-rich dwarf. If NSCs form at high redshift, they would inevitably be embedded in a gas-rich system. They conclude that star formation triggered by infalling gas-rich clusters dominates the mass of the resulting NSC, and considerably increases its density. The NSCs built in this way would fall near the high-mass end of the size-mass relation.

The work of Brown et al. (2018) is the only study to focus on in situ star formation using cosmological simulations. While these simulations run only to $z \sim 1.5$, they examine the effect of varying star formation efficiencies on the size, flattening, and rotation of the resulting cluster. They also examine the metallicity and abundances of stars formed, with the goal comparing it to the 'stripped NSC' candidate $\omega$ Cen (discussed further in Sect. 9). The extended star formation histories of these NSCs result in metallicity spreads of comparable to that in observed NSCs and the most massive globular clusters.

To summarize, there is strong evidence for in situ star formation happening in NSCs, and theoretical work shows that there are many ways for gas to reach the inner few parsec, both through merger activity and secular processes. However, setting up theoretical work suitable to compare to observations remains challenging. Hopefully, with increasing computational power and resolution, NSC formation can be more directly studied in galaxy-scale and cosmological simulations.

\subsection{NSC formation: different galaxies and different mechanisms}

The evidence reviewed above suggests that NSCs grow through both globular cluster inspiral and in situ formation. In this section, we tackle the question of which mechanism dominates the growth of NSCs in different types of hosts.

Apart from the aforementioned work of Hartmann et al. (2011) and Antonini et al. (2012) who argue that both formation mechanisms are required to replicate observations of specific NSCs, the only theoretical effort to tackle this problem has been the semi-analytic model of Antonini et al. (2015). This model includes prescriptions for both cluster infall and in situ formation, as well as a model for how central black holes influence NSC growth. They successfully predict the mass and high occupation of NSCs in galaxies at $\sim 10^{9} M_{\odot}$, and also the decline in occupation fractions at the highest masses due to the influence of black holes on the NSCs. Their model suggests that over most galaxy masses, $\sim 40 \%$ of the stars form 
in situ formation for both early and late types except for a higher fraction in the very highest mass early types $\left(M_{\star} \gtrsim 10^{11} M_{\odot}\right)$. Due to discreteness issues, their model fails to predict the drop in occupation fraction towards lower galaxies, (see Fig. 3). In addition, the model predicts a broadening and flattening in the galaxy massNSC mass correlation at high galaxy masses due to the ejection of NSC stars in massive black hole binary mergers. While a broadening does seem to occur at high masses (Fig. 12), if anything the mass-scaling relation appears to steepen rather than flattening (see discussion in Sect. 6.1).

Evidence for a transition at $M_{\star} \sim 10^{9} M_{\odot}$ : We have presented several pieces of evidence in this review that there appears to be a change in the growth mechanism of NSCs at galaxy masses of $\sim 10^{9} M_{\odot}$. We propose that at lower masses, NSCs grow primarily by globular cluster accretion, while at high masses, in situ formation becomes dominant. Based on Fig. 12 and Eq. 1, the NSC mass that corresponds to the transition at $M_{\star} \sim 10^{9} M_{\odot}$ is $M_{\mathrm{NSC}} \sim 3 \times 10^{6} M_{\odot}$. This transition is likely stochastic-i.e., some lower mass galaxies may experience a significant growth through in situ star formation. This suggestion has been initially discussed by Turner et al. (2012) and Sánchez-Janssen et al. (2019a), but the evidence collected here supports it more strongly. The evidence for the scenario is strongest for early-type galaxies, but is consistent with the evidence in late-type galaxies, as well. We enumerate the evidence in support of the scenario below, and then consider the challenges.

1. The first piece of evidence comes from the spectroscopic metallicities of earlytype galaxies, as shown in Fig. 9. It is clear in this figure that below $10^{9} \mathrm{M}_{\odot}$, about half of NSCs are more metal-poor than their surrounding hosts. This provides strong evidence for the importance of globular cluster accretion in this mass regime. At higher masses, most NSCs are more metal-rich than their NSCs, suggesting in situ star formation from gas enriched within the galaxy (Brown et al. 2018).

2. There is strong evidence for in situ star formation in late-type galaxies above $10^{9} M_{\odot}$ (Sect. 7.3); however, there is a lack of comparable data on lower mass late-type galaxies. If these lower mass galaxies also transition to having NSCs dominated by globular cluster inspiral, we would expect these NSCs to be old and metal-poor; this possibility is hinted at in the colors of NSCs in Georgiev et al. (2009).

3. The occupation fraction of NSCs (Fig. 3) increases at low masses as $M_{\star}^{1 / 4}$, exactly tracking the occupation fraction of non-nuclear globular clusters in early-type galaxies up until $\sim 10^{9} M_{\odot}$, where NSCs are nearly ubiquitous in all galaxies (Sánchez-Janssen et al. 2019a). Note that the NSC occupation fraction of early-type galaxies quickly declines above this mass, probably due to disruption or prevention from central black holes (Bekki and Graham 2010; Antonini et al. 2015).

4. The NSCs in the highest mass early-type galaxies are significantly flattened (Fig. 5; Spengler et al. 2017), similar to the disks of young stars seen in late- 
type NSCs (Seth et al. 2006) suggesting that their NSCs are grown (in the past) through in situ star formation.

5. At low masses, the scaling of NSC mass with galaxy mass is exactly in line with the predictions of globular cluster inspiral (Sect. 7.2; Gnedin et al. 2014; Sánchez-Janssen et al. 2019a). The mass function of NSCs shows some sign of steepening at the highest mass end (Sect. 6.1), as might be expected if a new source of growth (i.e., situ) becomes important.

6. There appears to be a break in the NSC size-mass relation (Fig. 7) at $M_{\mathrm{NSC}} \sim 3 \times 10^{6} M_{\odot}$, as might be expected if the growth mechanism was changing at this NSC mass.

While there is no strong evidence contradicting our proposal, there are several issues that may not be consistent with it. First of all, related to the last point raised, in Sect. 7.2, we noted that the slope of the mass-radius at the low-mass end is basically flat, and, thus, does not agree with the predictions from cluster merging simulations despite the other evidence that cluster merging dominates NSC growth at these masses. Second, it provides no clear guide as to why the NSC occupation fraction (at least for lower mass NSCs) appears to vary with environment (Sect. 4). Third, there is a lack of data in low-mass late-type galaxies that could support a transition in the dominant formation mechanism; the evidence all comes from the early types. We return to this lack of data and what further evidence could be collected to test this scenario in Sect. 11.

This proposed change of NSC growth mechanism at $\sim 10^{9} M_{\odot}$ also brings up the question of what is causing this change. If this change really occurs, some mechanism must prevent lower mass galaxies from effectively channeling or retaining gas in their nuclei. This could, therefore, be related to a change in the ability to form bars or to stellar feedback preventing formation in the nucleus. As discussed further in the next section, we have strong evidence for central black holes only for galaxies above $\sim 10^{9} M_{\odot}$, while below this mass, there are almost no clear detections. Therefore, it is possible that the change in NSC growth mechanisms at $\sim 10^{9} M_{\odot}$ is related to the presence of massive black holes. However, it is also possible that black hole growth is driven by the same gas reaching the center that forms the NSC through in situ formation.

\section{Nuclear star clusters and massive black holes}

Nuclear star clusters dominate the centers of galaxies with stellar masses in the range $\sim 10^{8}$ to $10^{10} M_{\odot}$ (see Fig. 3). Above this mass range, one invariably finds supermassive black holes (SMBHs) occupying the centers of massive galaxies (see recent review by Kormendy and Ho 2013). Interestingly, the masses of both SMBHs and NSCs follow a tight relation with the masses of their host galaxies (Wehner and Harris 2006; Rossa et al. 2006; Ferrarese et al. 2006a). This has led to speculations that NSCs take over the role of SMBHs in low-mass galaxies, leading to the term of 'central massive object' (CMO) to combine both incarnations of a seemingly similar 
product of galaxy evolution (Ferrarese et al. 2006a; Wehner and Harris 2006; Côté et al. 2006).

However, there are a number of cases where nuclear star clusters and massive black holes are found to co-exist (Filippenko and Ho 2003; Seth et al. 2008a; Graham and Spitler 2009; Neumayer and Walcher 2012; Nguyen et al. 2019). These include some of the earliest detected black holes, like M 31, M 32, NGC 3115, and the Milky Way (Tonry 1984, 1987; Dressler and Richstone 1988; Richstone et al. 1990; Bacon et al. 1994; Kormendy and Richstone 1992; van der Marel et al. 1994). The best-studied example by far is our own galaxy, where an SMBH with a mass of $4 \times 10^{6} M_{\odot}$ resides in an NSC with $\sim 3 \times 10^{7} M_{\odot}$ (Genzel et al. 2010; Schödel et al. 2014b; Feldmeier-Krause et al. 2017b, see also Sect. 5.6). A few more examples are well studied, and accurate masses for both the NSC and the BH are available. These findings indicate that NSCs are not an alternative incarnation of SMBHs, and refute the notion that NSCs replace massive black holes as the central massive object in low-mass galaxies. Instead, they suggest that the build-up of NSCs and the growth of massive black holes are closely related.

\subsection{Evidence for massive black holes within NSCs}

Most NSCs are readily observed in the images of nearby galaxies (see Fig. 1). The detection of supermassive black holes, on the other hand, is generally much harder, because it involves indirect detection techniques such as the characterisation of radiation from the black hole accretion disk, or kinematic measurements and dynamical modelling of the motion of gas or stars in the immediate vicinity of the black hole. This task is challenging even for the nearest galactic nuclei, and becomes ever more difficult with increasing distance and decreasing black hole mass.

In the closest example, the Milky Way, it is interesting to note that the NSC had been known (Becklin and Neugebauer 1968) before the SMBH was discovered (Balick and Brown 1974). The combined mass of both entities was measured using gas (Lacy et al. 1980, 1982) and stellar kinematics (Rieke and Rieke 1988; McGinn et al. 1989). However, it took more than 3 decades of hard work and vast technological improvement in observing facilities and instrumentation to achieve the high spatial resolution and wide-field coverage necessary to disentangle the relative masses of the SMBH and the NSC (see Genzel et al. 2010; Schödel et al. 2014b, for a recent review).

Other nearby examples for black hole detections within NSCs are M 31 (Bacon et al. 1994; Bender et al. 2005) and M 32 (Verolme et al. 2002; Nguyen et al. 2018). In contrast, M 33 hosts a well-studied NSC (Kormendy and McClure 1993; Kormendy et al. 2010), but shows no sign of a massive back hole (Gebhardt et al. 2001). The absence of a black hole in M 33 has led to the assumption that low-mass spiral galaxies without a bulge do not host massive black holes. This notion was turned upside down with the detection of an active black hole in the NSC of a bulgeless galaxy with a very similar stellar mass to M 33, NGC 4395 (Filippenko and Ho 2003). The mass of the black hole in NGC 4395 has been consistently measured to 
be $\sim 4 \times 10^{5} M_{\odot}$ using reverberation mapping as well as gas dynamical modelling (Peterson et al. 2005; den Brok et al. 2015), which, for a long time, constituted the lowest mass central black hole detection. ${ }^{5}$

The advancement of high spatial resolution observing techniques such as adaptive optics (Davies and Kasper 2012) has been fundamental in pushing the detection of massive black holes down to even lower masses. Low-mass black holes $\left(<10^{6} M_{\odot}\right.$ ) dominate the dynamics only within a very small volume (typically within $<1 \mathrm{pc}$ ), and one needs to spatially resolve this region to detect the effect of the black hole on its surroundings. In principle, the high stellar densities within NSCs are conducive to the dynamical detection of low-mass black holes, as they ensure an abundance of stellar orbits very close to the (putative) black hole, which, in turn, helps to reveal its dynamical signature (i.e., enhanced rotational signal and rise in the velocity dispersion). To separate the gravitational effect of the NSC from that of the black hole, accurate high-resolution mass models for the NSC are essential. Combining stellar population analysis with dynamical modelling has thereby enabled black hole mass detections below $\sim 10^{5} M_{\odot}$ in NSCs (Nguyen et al. 2019). Recent work on a volume limited sample of five $10^{9}-10^{10} M_{\odot}$ earlytype galaxies has shown that all have evidence for central black holes (Nguyen et al. 2017, 2018, 2019), providing strong evidence that at this mass range where NSC occupation peaks, many co-exist with massive black holes. While Nguyen et al. (2017) published only an upper limit on the black hole mass in NGC404, there is evidence for a massive black hole from AGN signatures (Seth et al. 2010; Nguyen et al. 2017, and references therein), and the recent ALMA observations of Davis et al. (2020) confirm this with a consistent stellar and gas dynamical detection of a massive black hole with $M_{\mathrm{BH}}=5.5_{-3.8}^{+4.1} \times 10^{5} M_{\odot}$.

In addition to dynamical constraints, the accretion signal in active galactic nuclei reveals the presence of central massive black holes in NSCs (Filippenko and Ho 2003; Seth et al. 2008a). Tailored surveys in the X-rays (Gallo et al. 2010; Mezcua et al. 2016, 2018), as well as studies of large samples in the optical (Reines et al. 2013; Baldassare et al. 2018) and infrared (Satyapal et al. 2008, 2009) have revealed a sizeable number of black holes in low-mass galaxies, although the fraction of galaxies with detected black holes at the low-mass end is quite small (see also Moran et al. 2014). An analysis of the X-ray detections in early-type galaxies suggests a high fraction of galaxies with black holes in galaxies with masses as low as $\sim 10^{9} M_{\odot}$ (Miller et al. 2015) in agreement with the dynamical results discussed above. In addition, AGN studies show similar detection fractions in early- and latetype nucleated galaxies (Foord et al. 2017; Birchall et al. 2020). Accretion observations have also revealed evidence for BHs below $10^{5} M_{\odot}$ (Baldassare et al. 2015; Chilingarian et al. 2018). Tidal disruption events have also started to uncover populations of lower mass galaxies; half of hosts have stellar masses below $10^{10} M_{\odot}$ (Law-Smith et al. 2017), and 75\% have dispersions below $100 \mathrm{~km} / \mathrm{s}$ (Wevers et al. 2017). Comparison of the galaxy demographics for observed tidal disruption events

\footnotetext{
5 Note that a recent study by Woo et al. (2019) claims an even lower mass of $\sim 10^{4} M_{\odot}$ for the massive black hole in NGC 4395.
} 
has the promise of unveiling the occupation fraction of black holes in low-mass galaxies (e.g., Stone and Metzger 2016; van Velzen 2018). Currently, there is little evidence for central massive black holes in galaxies below $M_{\star} \sim 10^{9} M_{\odot}$, although a handful of candidates do exist (e.g., Maksym et al. 2014; Wevers et al. 2019; Reines et al. 2019).

Black holes of even lower masses (crossing into the realm of intermediate-mass BHs) have been claimed to exist at the centers of the likely stripped NSCs G1 (Gebhardt et al. 2002), $\omega$ Cen (Noyola et al. 2010), and M 54 (Ibata et al. 2009; Baumgardt 2017). These dynamical detections are still debated (e.g., van der Marel and Anderson 2010; Miller-Jones et al. 2012; Tremou et al. 2018; Baumgardt et al. 2019), and further work at even higher spatial resolution is needed to reliably confirm or reject intermediate-mass black holes in these objects. The recent review by Greene et al. (2019) provides an in-depth discussion of the reliability of globular cluster black hole measurements, as well as a table of low-mass black hole candidates and their overlap with NSCs.

\subsection{Co-existence of NSCs and massive black holes}

As outlined in the last section, NSCs and massive black holes are found to co-exist in many galaxies, especially in the host galaxy mass range $10^{9}-10^{10} M_{\odot}$. Towards higher host galaxy masses, NSCs are less common, and their demise in galaxies of masses above $\sim 10^{11} M_{\odot}$ (see Fig. 3) might be related to the presence and the dynamical interaction with the massive black hole (Antonini et al. 2015).

If the growth of the massive black hole and the NSC are, indeed, interrelated, their mass ratio might be an interesting metric to study. Several authors have investigated this quantity (Graham and Spitler 2009; Seth et al. 2008a; Neumayer and Walcher 2012; Georgiev et al. 2016; Nguyen et al. 2018), and we present an extensive compilation in Fig. 13 and Table 3, which suggests that the mass fraction $M_{\mathrm{BH}} / M_{\mathrm{NSC}}$ increases with host galaxy mass. However, the scatter in this ratio at a given galaxy mass is still up to three orders of magnitude. This likely reflects the fact that the growth history of both NSC and BH is very stochastic.

The host galaxy mass at which $\mathrm{BHs}$ start to dominate (the ratio $M_{\mathrm{BH}} / M_{\mathrm{NSC}}$ becomes $>1$ ) is a few times $10^{10} M_{\odot}$, corresponding to a host galaxy velocity dispersion of $>100 \mathrm{~km} / \mathrm{s}$. Nayakshin et al. (2009) find that the changeover from NSCs to SMBHs occurs when the dynamical time is about equal to the Salpeter time, i.e., the time over which the black hole mass doubles. In other words, lowdispersion galaxies with dynamical times shorter than the Salpeter time cannot grow their central massive black holes quickly enough to affect the gas infall, and this gas accumulates at the center and will form NSCs. These galaxies might still host massive black holes, but they would be underweight with respect to their host galaxy mass. An alternative approach to explaining the dividing line between NSCand SMBH-dominated galactic nuclei was given by Stone et al. (2017) who show that the low-mass end of the SMBH mass function can be explained by collisions of stellar mass black holes and subsequent runaway tidal capture and tidal disruption 

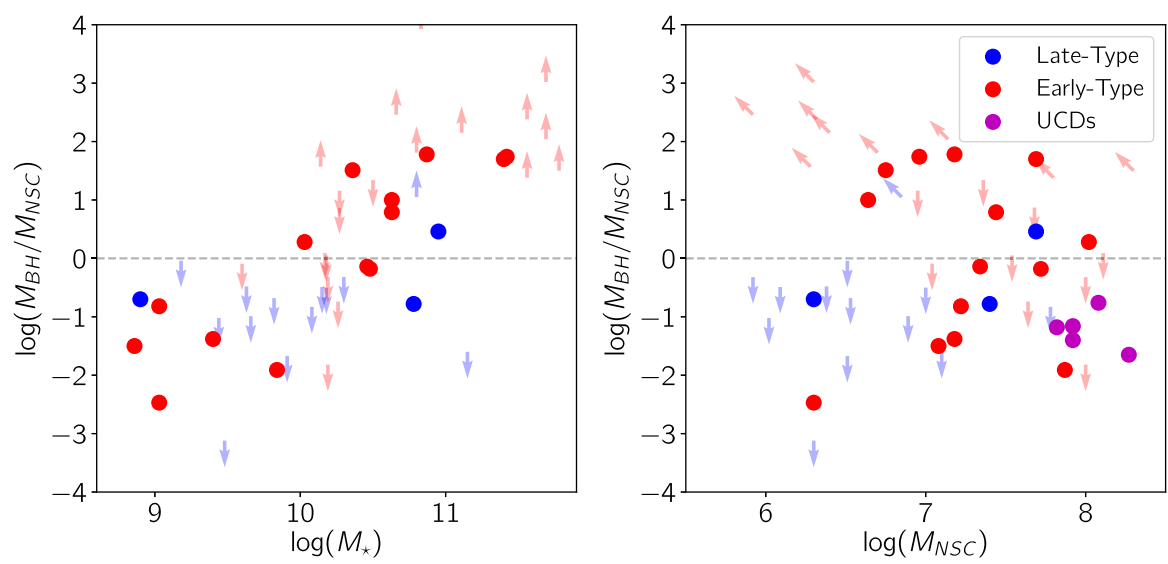

Fig. 13 The ratio of the masses of the central massive black hole and the NSC plotted against their host galaxy stellar mass (left) and against the NSC dynamical mass (right). The data plotted are compiled in Table 3. The dashed line indicates equal mass NSCs and black holes in galaxies, while objects above the line have more massive black holes than NSCs. Coloring breaks the galaxies into early-type (red), latetype (blue), and UCDs/stripped galaxy nuclei (purple; plotted only in the right-hand plot). Upper and lower limits result from objects with upper limits on black hole masses and NSC masses, respectively. This plot builds on compilations presented in several previous works (Seth et al. 2008a; Graham and Spitler 2009; Neumayer and Walcher 2012; Georgiev et al. 2016; Nguyen et al. 2018)

events in NSCs. We will discuss the formation channel of massive black holes in the next section.

\subsection{Massive black hole formation and growth within NSCs}

The formation of SMBHs remains a mystery, and is, therefore, a topic of vast current interest. Several recent reviews summarise the different proposed formation channels (Volonteri 2010, 2012; Mezcua 2017; Greene et al. 2019; Inayoshi et al. 2019). Here, we focus on one of the proposed channels, namely the formation and runaway collisions of black holes within dense (nuclear) star clusters. The idea for the formation of an (intermediate mass) black hole within a dense stellar cluster has been discussed for a long time (Begelman and Rees 1978; Quinlan and Shapiro 1987; Ebisuzaki et al. 2001; Miller and Hamilton 2002; Portegies Zwart and McMillan 2002; Portegies Zwart et al. 2004; Gürkan et al. 2004; Freitag et al. $2006 a, b)$. The most massive and dense NSCs have high enough velocity dispersions that binary heating cannot prevent core collapse, and this can result in the formation of massive black holes (Miller and Davies 2012). However, lower mass NSCs (typically in lower mass galaxies) fall below this threshold.

Antonini et al. (2019) point out that in a star cluster with a sufficiently large escape velocity, black holes that are produced by black hole mergers can be retained, merge again, and grow to larger masses. This means that runaway collisions are favoured in the most massive clusters, as they can retain black holes against the gravitational recoil kicks produced during the merger of two black holes (Holley-Bockelmann et al. 2008). While NSCs are the densest stellar systems 
Table 3 Data and references for Fig. 13

\begin{tabular}{|c|c|c|c|c|c|c|c|}
\hline $\begin{array}{l}\text { (1) } \\
\text { Galaxy }\end{array}$ & $\begin{array}{l}\text { (2) } \\
\text { Type }\end{array}$ & $\begin{array}{l}(3) \\
\log \left(M_{\mathrm{BH}}\right)\end{array}$ & $\begin{array}{l}\text { (4) } \\
\text { Ref. }\end{array}$ & $\begin{array}{l}(5) \\
\log \left(M_{\mathrm{NSC}}\right)\end{array}$ & $\begin{array}{l}\text { (6) } \\
\text { Ref. }\end{array}$ & $\begin{array}{l}(7) \\
\log \left(M_{\text {gal }}\right)\end{array}$ & $\begin{array}{l}\text { (8) } \\
\text { Ref. }\end{array}$ \\
\hline \multicolumn{8}{|c|}{ Objects with NSCs and BH masses } \\
\hline Milky Way & Late & 6.62 & 1,2 & 7.40 & 34 & 10.78 & 42 \\
\hline M 31 & Late & 8.15 & 3 & 7.69 & 35 & 10.95 & 43 \\
\hline NGC 205 & Early & 3.83 & 4 & 6.30 & 5 & 9.03 & 5 \\
\hline M 32 & Early & 6.40 & 5 & 7.22 & 5 & 9.03 & 5 \\
\hline NGC 404 & Early & 5.58 & 6 & 7.08 & 36 & 8.86 & 44 \\
\hline NGC 5102 & Early & 5.96 & 4 & 7.87 & 5 & 9.84 & 5 \\
\hline NGC 5206 & Early & 5.80 & 4 & 7.18 & 5 & 9.40 & 5 \\
\hline NGC 4395 & Late & 5.60 & 7,8 & 6.30 & 8 & 8.90 & 45 \\
\hline M 60-UCD1 & UCD & 7.32 & 9 & 8.08 & 9 & - & - \\
\hline M 59cO & UCD & 6.76 & 10 & 7.92 & 10 & - & - \\
\hline VUCD3 & UCD & 6.64 & 10 & 7.82 & 10 & - & - \\
\hline M 59-UCD3 & UCD & 6.62 & 11 & 8.27 & 11 & - & - \\
\hline FUCD3 & UCD & 6.52 & 12 & 7.92 & 12 & - & - \\
\hline NGC 4578 & Early & 7.54 & 13 & 7.72 & 37 & 10.48 & 46 \\
\hline NGC 1023 & Early & 7.64 & 14 & 6.64 & 38 & 10.63 & 45 \\
\hline NGC 3115 & Early & 8.96 & 15 & 7.18 & 38 & 10.87 & 38 \\
\hline NGC 3384 & Early & 7.20 & 16 & 7.34 & 38 & 10.46 & 45 \\
\hline NGC 4026 & Early & 8.26 & 17 & 6.75 & 38 & 10.36 & 18 \\
\hline NGC 4697 & Early & 8.23 & 16 & 7.44 & 38 & 10.63 & 45 \\
\hline IC 1459 & Early & 9.39 & 18 & 7.69 & 39 & 11.40 & 18 \\
\hline NGC 4552 & Early & 8.70 & 16 & 6.96 & 39 & 11.42 & 18 \\
\hline NGC 4558/VCC1146 & Early & 8.30 & 19 & 8.02 & 40 & 10.03 & 46 \\
\hline \multicolumn{8}{|c|}{ Objects with NSC masses and BH upper limits } \\
\hline M 33 & Late & $<3.18$ & 20 & 6.30 & 38 & 9.48 & 47 \\
\hline NGC 4474/VCC 1242 & Early & $<6.18$ & 13 & 8.0 & 40 & 10.19 & 46 \\
\hline NGC 4551/VCC 1630 & Early & $<6.90$ & 13 & 7.64 & 40 & 10.26 & 46 \\
\hline NGC 300 & Late & $<5.00$ & 21 & 6.02 & 41 & 9.44 & 5 \\
\hline NGC 428 & Late & $<4.84$ & 21 & 6.51 & 41 & 9.91 & 5 \\
\hline NGC 1042 & Late & $<6.47$ & 21 & 6.51 & 41 & 9.18 & 5 \\
\hline NGC 1493 & Late & $<5.90$ & 21 & 6.38 & 41 & 9.63 & 5 \\
\hline NGC 2139 & Late & $<5.60$ & 21 & 5.92 & 41 & 10.30 & 5 \\
\hline NGC 3423 & Late & $<5.85$ & 21 & 6.53 & 41 & 9.82 & 5 \\
\hline NGC 7418 & Late & $<6.95$ & 21 & 7.78 & 41 & 10.08 & 5 \\
\hline NGC 7424 & Late & $<5.60$ & 21 & 6.09 & 41 & 10.15 & 5 \\
\hline NGC 7793 & Late & $<5.90$ & 21 & 6.89 & 41 & 9.66 & 5 \\
\hline VCC 1254 & Early & $<6.95$ & 22 & 7.04 & 38 & 9.60 & 38 \\
\hline NGC 3621 & Late & $<6.50$ & 23 & 7.00 & 23 & 10.18 & 48 \\
\hline IC 342 & Late & $<5.50$ & 24 & 7.1 & 24 & 11.15 & 49 \\
\hline NGC 2778 & Early & $<8.70$ & 19 & 7.36 & 39 & 10.50 & 46 \\
\hline NGC 4379/VCC 784 & Early & $<8.55$ & 19 & 7.68 & 40 & 10.27 & 46 \\
\hline
\end{tabular}


Table 3 continued

\begin{tabular}{|c|c|c|c|c|c|c|c|}
\hline $\begin{array}{l}\text { (1) } \\
\text { Galaxy }\end{array}$ & $\begin{array}{l}\text { (2) } \\
\text { Type }\end{array}$ & $\begin{array}{l}(3) \\
\log \left(M_{\mathrm{BH}}\right)\end{array}$ & $\begin{array}{l}\text { (4) } \\
\text { Ref. }\end{array}$ & $\begin{array}{l}(5) \\
\log \left(M_{\mathrm{NSC}}\right)\end{array}$ & $\begin{array}{l}\text { (6) } \\
\text { Ref. }\end{array}$ & $\begin{array}{l}(7) \\
\log \left(M_{\mathrm{gal}}\right)\end{array}$ & $\begin{array}{l}\text { (8) } \\
\text { Ref. }\end{array}$ \\
\hline NGC $4387 /$ VCC 828 & Early & $<7.59$ & 19 & 7.54 & 40 & 10.18 & 46 \\
\hline NGC 4474/VCC 1242 & Early & $<7.68$ & 19 & 8.00 & 40 & 10.19 & 46 \\
\hline NGC 4612/VCC 1883 & Early & $<8.11$ & 19 & 6.95 & 40 & 10.27 & 46 \\
\hline NGC 4623/VCC 1913 & Early & $<8.20$ & 19 & 8.11 & 37 & 10.17 & 46 \\
\hline \multicolumn{8}{|c|}{ Objects with BH masses and NSC upper limits } \\
\hline NGC 4486 & Early & 9.80 & 25 & $<8.30$ & 21 & 11.78 & 21 \\
\hline NGC 4374 & Early & 9.18 & 26 & $<7.80$ & 21 & 11.56 & 21 \\
\hline NGC 1332 & Early & 9.18 & 27 & $<7.14$ & 21 & 11.69 & 5 \\
\hline NGC 3031 & Late & 7.90 & 28 & $<6.85$ & 21 & 10.80 & 50 \\
\hline NGC 4261 & Early & 8.70 & 29 & $<6.32$ & 21 & 11.56 & 21 \\
\hline NGC 4649 & Early & 9.32 & 16 & $<6.30$ & 21 & 11.69 & 21 \\
\hline NGC 3998 & Early & 8.38 & 30 & $<5.92$ & 21 & 10.66 & 51 \\
\hline NGC 2787 & Early & 7.85 & 31 & $<6.28$ & 21 & 10.14 & 51 \\
\hline NGC 3379 & Early & 8.08 & 32 & $<4.15$ & 21 & 10.83 & 21 \\
\hline NGC 4342 & Early & 8.55 & 33 & $<6.40$ & 21 & 11.11 & 21 \\
\hline NGC 4291 & Early & 8.51 & 16 & $<6.70$ & 21 & 10.80 & 21 \\
\hline
\end{tabular}

(1), (2): Galaxy name and morphological type. (3), (4): logarithm of black hole mass and assoc. literature reference. (5), (6): logarithm of NSC mass and reference. (7), (8): logarithm of galaxy stellar mass and reference

References-(1) Gravity Collaboration et al. (2019), (2) Do et al. (2019), (3) Bender et al. (2005), (4) Nguyen et al. (2019), (5) Nguyen et al. (2018), (6) Davis et al. in prep., (7) Peterson et al. (2005), (8) den Brok et al. (2015), (9) Seth et al. (2014), (10) Ahn et al. (2017), (11) Ahn et al. (2018), (12) Afanasiev et al. (2018), (13) Krajnović et al. (2018), (14) Bower et al. (2001), (15) Emsellem et al. (1999), (16) Gebhardt et al. (2003), (17) Gültekin et al. (2009), (18) Saglia et al. (2016), (19) Pechetti et al. (2017), (20) Gebhardt et al. (2001), (21) Neumayer and Walcher (2012), (22) Geha et al. (2002), (23) Barth et al. (2009), (24) Böker et al. (1999), (25) Gebhardt et al. (2011), (26) Bower et al. (1998), (27) Rusli et al. (2011), (28) Devereux et al. (2003), (29) Ferrarese et al. (1996), (30) de Francesco et al. (2006), (31) Sarzi et al. (2001), (32) Shapiro et al. (2006), (33) Cretton and van den Bosch (1999), (34) Schödel et al. (2014a), (35) Peng (2002), (36) Nguyen et al. (2017), (37) Côté et al. (2006), (38) Graham and Spitler (2009), (39) Lauer et al. (2005), (40) Spengler et al. (2017), (41) Walcher et al. (2005), (42) BlandHawthorn and Gerhard (2016), (43) Williams et al. (2017), (44) Seth et al. (2010), (45) Reines and Volonteri (2015), (46) Cappellari et al. (2013), (47) McConnachie (2012), (48) derived from Jarrett et al. (2003), (49) Karachentsev et al. (2013), (50) Querejeta et al. (2015), (51) derived from Jarrett et al. (2000)

known in the local universe, even denser clusters may have existed at high redshift, with central subclusters driven to even higher densities through gas dissipational processes (Davies et al. 2011; Leigh et al. 2014). These would be even more susceptible to the early formation of massive black holes.

The subsequent growth of the massive black hole can be sustained through different channels. As mentioned in Sects. 5.4 and 7.3, many NSCs experience periodic bursts of star formation (Walcher et al. 2006; Carson et al. 2015; Kacharov et al. 2018). It is likely that the same gas that is feeding star formation also feeds the black hole. In that sense, the presence of an NSC may, in fact, enhance the growth rate of the central black hole (Naiman et al. 2015). This notion is supported by the 
simulations of Hopkins and Quataert (2010a) who find that nuclear star formation is more tightly coupled to the growth of central black holes than the global star formation rate.

As discussed in the previous subsection, another growth channel for the central massive black hole is via the tidal disruption and capture of stars coming close to the central black hole (e.g., Strubbe and Quataert 2009). This is especially true for lower mass black holes $\left(<2 \times 10^{6} M_{\odot}\right)$, which may acquire the majority of mass through tidal capture (Milosavljević et al. 2006). However, in the lowest mass galaxies (below $\sigma \sim 35 \mathrm{~km} / \mathrm{s}$ ), NSCs are not dense enough to fuel black hole growth by runaway tidal capture of NSC stars (Miller and Davies 2012; Stone et al. 2017). This may be related to the lack of massive central black holes in the lowest mass galaxies.

\section{Stripped down: connecting nuclear star clusters, globular clusters, and ultra-compact dwarf galaxies}

Given the hierarchical nature of galaxy formation, smaller galaxies often fall into larger galaxies, and are tidally disrupted in the process. The gravitationally selfbound NSCs in these galaxies are likely to survive this disruption process (Bassino et al. 1994; Bekki et al. 2003; Pfeffer and Baumgardt 2013) and will continue their lives orbiting in the halo of the larger galaxy. This process is currently ongoing in the Sgr dSph galaxy, whose NSC was first discovered as the globular cluster M 54. In this section, we discuss the evidence for stripped NSCs, both outside our Milky Way and hidden amongst the Milky Way's globular cluster system. These stripped NSCs can be important for: (1) understanding the formation and evolution of NSCs, (2) preserving a record of a galaxy's accretion history, and (3) obtaining a census of massive black holes.

\subsection{Extragalactic evidence for stripped NSCs: ultra-compact dwarfs}

Outside the Milky Way, evidence for the survival of nuclei after tidal disruption is found in the existence of ultra-compact dwarfs (UCDs), a class of objects that constitutes the brightest compact stellar systems (Hilker et al. 1999; Drinkwater et al. 2000). In the size-mass plane, no clear boundary exists between "ordinary" massive globular clusters and UCDs, while NSCs and UCDs appear in overlapping regions (see Fig. 7; Norris et al. 2014). Identifying which UCDs are stripped NSCs is, therefore, a complicated task, a difficulty that is enhanced by our current lack of understanding of the globular cluster formation process. Stripped NSCs have been identified through the presence of (1) an extended SFH, (2) a massive black hole, or (3) a significant halo or stream of tidal material. None of these signatures are expected for ordinary globular clusters. ${ }^{6}$ However, not all stripped NSCs are expected to have these signatures, and obtaining a census of all stripped NSCs,

\footnotetext{
6 Throughout this section, we will refer to ordinary globular clusters as massive clusters that were not once NSCs.
} 
therefore, is challenging. In what follows, we summarize the observational evidence for (some) UCDs being stripped NSCs.

The extended SFH of many NSCs has been discussed in Sect. 5.4. For tidally stripped systems, in addition to the extended SFH in M 54, a clearly extended SFH has been found in NGC4546-UCD1 (Norris et al. 2015). In several other UCDs, a younger age or higher metallicity than typical GCs is inferred from single stellar population modeling, suggesting that they also are stripped NSCs (Janz et al. 2016).

As discussed in Sect. 8, BHs with masses that are a significant fraction of their NSC mass are common in massive NSCs. In UCDs, detection of these black holes can provide strong evidence for stripping. Mieske et al. (2013) proposed that the inflated dynamical mass estimates seen in many UCDs could be explained with black holes of order $10 \%$ of the total UCD mass. Adaptive optic observations have now found evidence for BHs with mass fractions of 2-18\% in all five UCDs searched with luminosities above $10^{7} L_{\odot}$ (Seth et al. 2014; Ahn et al. 2017, 2018; Afanasiev et al. 2018). By applying the well-known scaling relations between BH and host galaxy, the $\mathrm{BH}$ masses can provide additional insight into the UCD progenitor galaxies. On the other hand, in less luminous systems, BHs have been more elusive (e.g., Gebhardt et al. 2005; Voggel et al. 2018) despite the dynamical BH detections in some lower luminosity NSCs (Nguyen et al. 2018, 2019). With the evidence of inflated dynamical mass estimates being an indicator of BHs, the fraction of UCDs with dynamical evidence for the presence of a massive $\mathrm{BH}$ from integrated spectroscopy has recently been reanalyzed, showing a rise from $\sim 20 \%$ at $10^{6} L_{\odot}$ to $\sim 70 \%$ at the highest luminosities (Voggel et al. 2019).

Simulations by Pfeffer and Baumgardt (2013) suggest that NSC properties are mostly unchanged during tidal stripping, but that a remnant halo (or stream) of stars from the stripped galaxy may surround these NSCs for an extended period of time. Such halos have, indeed, been detected in a number of cases, ranging from sources embedded in tidal streams (Jennings et al. 2015; Voggel et al. 2016) to extended halos and multiple component fits being required to model the surface brightness profiles of UCDs (e.g., Martini and Ho 2004; Evstigneeva et al. 2008; Chilingarian and Mamon 2008; Strader et al. 2013; Liu et al. 2015; Ahn et al. 2017).

\subsection{Abundance of stripped NSCs}

Overall, there are multiple lines of evidence, suggesting that many massive UCDs are in fact stripped NSCs. Two related questions to this are (1) what fraction of the UCD population are stripped NSCs, and (2) what is the relative number of "presentday" NSCs (i.e., that still occupy their host nucleus) to the number of 'former' NSCs from stripped galaxies. Tackling these questions from the theoretical side using semi-analytic methods based on the Millenium-II simulations, Pfeffer et al. (2014, 2016) suggest that stripped NSCs can account for all UCDs observed in the Virgo and Fornax cluster above a mass of $\sim 2 \times 10^{7} M_{\odot}$, while only a small fraction of lower mass UCDs are likely to be stripped NSCs. However, accurately tracking the fate of infalling subhalos within N-body simulations has shown to be 
challenging (e.g., van den Bosch et al. 2018), suggesting that there is a significant uncertainty in predicting which satellites are disrupted and which are not.

From the observational side, Ferrarese et al. (2016) show that, assuming that all UCDs in Virgo are stripped NSCs, the number of stripped NSCs outnumbers the number of the present-day NSCs by a factor of $\sim 3$. Taking into account only those UCDs with dynamical evidence for massive black holes, Voggel et al. (2019) estimates that the ratio of stripped NSCs to the present-day NSCs in nearby galaxy clusters is $\sim 1$. Furthermore, they show that this ratio depends on the luminosity of the NSCs/UCDs (and, therefore, the stellar masses of the host galaxies), in the sense that stripped NSCs are more abundant at lower luminosity. Given that some stripped NSCs may lack massive black holes, the true ratio of stripped NSCs to the presentday NSCs in clusters likely falls between one and three.

The ratio of stripped to the present-day NSCs may also be dependent on the galaxy environment, with the Milky Way providing a useful test bed for predictions in lower mass halos. Pfeffer et al. (2014) suggest that 1-3 stripped NSCs may be hidden amongst the globular cluster population. Using a different approach to simulate the formation of massive clusters based on a suite of "zoomed-in" SPH simulations, Kruijssen et al. (2019) suggest $\sim 6$ stripped NSCs in the Milky Way. We examine the observational evidence for stripped NSCs in the Milky Way in the next subsection.

\subsection{Stripped NSCs amongst Milky Way globular clusters}

The brightest, most massive globular clusters in the Milky Way, $\omega$ Cen has long been suspected to be a former NSC (e.g., Bekki and Freeman 2003), due to its large spread in metallicity (e.g., Norris et al. 1996; Johnson and Pilachowski 2010), and possibly also an age spread of several Gyr (Hilker et al. 2004; Villanova et al. 2014). However, this age spread is difficult to interpret, due to the fact that the abundance spreads seen in many stars can also affect their age estimates (e.g., Marino et al. 2012). However, $\omega$ Cen's status as a stripped NSC has recently been significantly strengthened through the identification of material that may be associated with its progenitor galaxy (Majewski et al. 2012; Ibata et al. 2019). As discussed in Sect. 5.4, M 54, the NSC of the Sgr dwarf galaxy, shares many similarities with $\omega$ Cen, suggesting that there are at least two very strong candidates for (partially) stripped NSCs amongst the Milky Way's globular clusters.

Moreover, the complex stellar population of $\omega$ Cen and M 54 are becoming less and less unique as the Milky Way's GC system is being studied in more detail, with metallicity spreads now found in at least nine clusters (Marino et al. 2015; Da Costa 2016). An overlapping set of clusters show color-magnitude and color-color diagrams similar to that of $\omega$ Cen, also suggesting metallicity spreads (Type II clusters in Milone et al. 2017). If these globular clusters are, indeed, all stripped NSCs, it would suggest $\sim 10$ stripped NSCs, exceeding the model predictions of Pfeffer et al. (2014) and Kruijssen et al. (2019).

Despite the wealth of information available for Milky Way globular clusters, identifying these objects as stripped NSCs remains challenging. This is at least in part due to the range of likely formation mechanisms for NSCs. It is feasible to 
produce a stripped NSC with no spread in age or metallicity (e.g., a single cluster inspiraling to the nucleus or single star formation event), a spread in metallicity, but not age (e.g., dynamical friction inspiral of two ancient globular clusters with different metallicities), or a spread in both (e.g., inspiral of globular cluster(s) followed by in situ star formation events). Note that only in the latter case do the stellar populations of a stripped NSC provide clear evidence of its origin, and thus, an improved understanding of age spreads in globular clusters (including their complex chemical variations) seems like the next step required to obtain at least a lower limit on the number of stripped NSCs in the Milky Way.

\section{The unique astrophysics of very dense stellar systems}

The extreme stellar densities of NSCs $\left(>10^{6} M_{\odot} / \mathrm{pc}^{3}\right.$; Sect. 5.3), and their coincidence with massive black holes (Sect. 8) make NSCs an interesting laboratory. In the following section, we briefly highlight some of the work done on the unique dynamics of NSCs, as well as the consequences of these dynamics for unusual stellar populations, tidal disruption events, and compact binary systems that may emit gravitational waves.

\subsection{Stellar dynamical evolution of NSCs}

Due to their higher masses and larger sizes, the dynamical evolution of NSCs is slower than the evolution of typical globular clusters. Half-mass relaxation times are many gigayears, with the larger, more massive NSCs like the one in the Milky Way having $>10$ Gyr relaxation time at the half mass radius (e.g., Merritt 2009; Panamarev et al. 2019). The unique location of NSCs at the centers of galaxies means that, in addition to their internal dynamical evolution, they can be dynamically heated by both their surrounding galaxy and the massive black holes at their centers. Merritt (2009) suggests that two regimes exist: (1) lower mass galaxies have compact enough clusters to core collapse despite the energy input from the surrounding galaxies, while (2) in higher mass galaxies, a little dynamical evolution seems to be occurring due to their long relaxation times. Dynamical heating from binaries can also be important in NSCs that lack massive black holes (e.g., Antonini et al. 2019).

The presence of a central massive black hole within an NSC should lead to the formation of a cusp surrounding the massive black hole with a density slope of $\rho \propto r^{-\gamma}$ with $\gamma=7 / 4$ in single-mass models (Bahcall and Wolf 1976). The presence or absence of this Bahcall-Wolf cusp in NSCs, especially the Milky Way NSC, is debated in both the theoretical (e.g., Merritt 2010; Vasiliev 2017) and observational literature (e.g., Buchholz et al. 2009; Schödel et al. 2014a; Gallego-Cano et al. 2018). The current theoretical literature (Vasiliev 2017; Baumgardt et al. 2018; Panamarev et al. 2019) suggests that the mass segregation of stellar mass black holes towards the center leads to a steep cusp $(\gamma>1.5)$ in their distribution, while the stellar distribution remains somewhat flatter. This seems to be consistent with 
more recent observations that include fainter stars and unresolved light at the center (Gallego-Cano et al. 2018; Schödel et al. 2018).

\subsection{Nuclear star formation and stellar collisions}

As discussed in Sect. 5, the NSCs in the Milky Way, M 31, and many other galaxies seem to have concentrated young stellar populations at their centers. The presence of young stars is challenging to explain, as infalling young star clusters would disrupt at larger radii than they are observed (e.g., Gerhard 2001; Fujii et al. 2009). This suggests that in situ star formation is taking place, despite the disruptive tidal shear forces present at these small radii (e.g., Levin and Beloborodov 2003; Mapelli et al. 2012). Another possibility for the formation of the apparently young ( $\sim 200$ Myr) stars in M 31 and the very young stars in M 33 is that they are collisioninduced blue stragglers that form due to the high stellar density (Kormendy and McClure 1993; Demarque and Virani 2007; Leigh et al. 2016).

In addition to the presence of a young stellar component very close to the SMBH, another puzzling phenomenon is the apparent absence of red giant branch (RGB) stars near the center of the Milky Way (e.g., Do et al. 2009). This has been hypothesized to be a result of stellar collisions (Davies et al. 1998; Dale et al. 2009), although it is unclear whether the stellar/black hole density is high enough to effectively destroy the stars (Leigh et al. 2016). Other possible explanations are that the lack of RGB stars was caused by the destruction of these stars as they passed through a star-forming disk such as the one that created the young stars (AmaroSeoane and Chen 2014; Kieffer and Bogdanović 2016; Amaro-Seoane et al. 2020), or that the central region of the Milky Way NSC has not yet had enough time to form a cusp (Merritt 2010; Antonini 2014).

\subsection{Tidal disruption events and SMBH growth}

Tidal disruption events (TDEs) are a natural consequence of having a dense NSC surrounding a massive black hole. A TDE occurs when a star approaches, the SMBH to within less than its tidal radius. ${ }^{7}$ The star is then pulled apart by the tidal forces imparted by the $\mathrm{BH}$; part of its material is ejected away, while part is accreted onto the SMBH (e.g., Rees 1988).

The observation of TDEs is a rapidly growing field with detections at optical, UV, and X-ray wavelengths (see Auchettl et al. 2017, for a recent compilation of events). These TDEs are occurring in relatively low-mass galaxies, with nearly half of them having stellar masses between $10^{9}$ and $10^{10} M_{\odot}$ (Wevers et al. 2019), a mass range in which NSCs are ubiquitous. An important aspect of understanding the observed targets is predictions of TDE rates in galaxies, which strongly depend on the density of stars within the sphere of influence of the black hole (e.g., Stone and Metzger 2016). Therefore, the density profiles of NSCs are important for understanding the demographics of TDEs and the underlying black hole population

\footnotetext{
7 Approximately given by $r_{t}=(M / m)^{1 / 3} \times r$, where $M$ is the mass of the SMBH, $m$ is the mass of the star, and $r$ is the stellar radius.
} 
in galaxies. The formation mechanism of NSCs may also affect TDE rates, as merging of clusters or infall of gas could enhance TDE rates (Aharon et al. 2016; Lezhnin and Vasiliev 2016; Arca-Sedda and Capuzzo-Dolcetta 2017). Furthermore, as noted in Sect. 8, the NSC can be an important source of mass growth for black holes over time (Stone et al. 2017). A better understanding of NSC formation and density profiles is needed to quantify the TDE-induced $\mathrm{BH}$ growth throughout cosmic history, and to constrain the expected TDE detection rates for future timeresolved survey telescopes such as the Large Synoptic Survey Telescope (LSST).

\subsection{NSCs as gravitational-wave emitters}

The recent detection of gravitational-wave (GW) emission from merging stellar mass black holes (Abbott et al. 2016, 2019) has highlighted the role of galactic nuclei as potential sources for GW emission. Given their high stellar densities and escape velocities, NSCs are prime candidates for hosting dynamically created compact object mergers that can result in detectable LIGO sources. Stellar mass BH binary mergers appear to be more efficiently produced in more massive clusters and in younger clusters, thus favoring production in NSCs (e.g., Askar et al. 2018). The high mass of NSCs means that there is a higher expected retention of stellar mass BHs after supernova kicks (Miller and Lauburg 2009). Furthermore, stellar mass BHs also can experience successive mergers in NSCs, since a merged $\mathrm{BH}$ can be retained despite gravitational-wave recoil during the merger (e.g., Antonini et al. 2019). In NSCs lacking a central massive BH, stellar mass BHs will mass segregate to the center of the cluster and then can form binaries and harden through threebody interactions (e.g., Antonini and Rasio 2016; Choksi et al. 2019). The presence of a massive $\mathrm{BH}$ also enables stellar mass $\mathrm{BH}$ binaries to harden through KozaiLidov interactions with the massive BH (e.g., Antonini and Perets 2012; Zhang et al. 2019; Bub and Petrovich 2020), or via gas torques and/or hardening encounters with single stars in AGN disks (McKernan et al. 2018; Leigh et al. 2018). The presence of gas can even facilitate the formation of compact object mergers via dissipative effects (Secunda et al. 2019). In addition to stellar mass BH binary mergers, NSCs can also be a possible site of triple mergers of BHs and neutron stars (Fragione et al. 2019).

Lower frequency GW events will be detected in the future with LISA, and many of the events expected to be detected will be in NSCs (Amaro-Seoane et al. 2017). For instance, extreme mass-ratio inspirals (EMRIs) occur when a compact stellar remnant (BH or neutron star) falls into a massive BH (e.g., Hopman and Alexander 2006; Merritt et al. 2011). Similar to the TDE rates, the formation scenarios of NSCs and their subsequent dynamical evolution can significantly impact EMRI rates (Aharon and Perets 2016). Current rate estimates suggest that LISA should be able to detect multiple EMRIs at any given time (Emami and Loeb 2019). NSCs also likely play a role in the "final-parsec" problem that needs to be overcome for massive BH binaries to merge (e.g., Vasiliev et al. 2015; Khan et al. 2020). Overall, it is clear that the density profiles, morphology, and kinematics of NSCs play an important role in many types of GW events. 


\section{Summary, open issues, and outlook}

The hundreds of papers which we have discussed here have elevated our understanding of NSCs from merely bright points of light at the centers of nearby galaxies to the extremely dense and complex stellar systems which we now know them to be.

Still, in the process of writing this review, we have found a number of topics where our present knowledge is lacking. Perhaps, the most pressing need is for theoretical work on NSC formation. This includes simulations of NSC formation in the cosmological context, especially in the early universe. The simulations targeting the formation of the first stars and black holes (e.g., Wise et al. 2019), and the formation of globular clusters in the early Universe (e.g., Li et al. 2017; Ma et al. 2019) should provide us with valuable insight on the early epochs of NSC formation, and whether seed clusters or central black holes play a significant role in promoting the formation of NSCs. Also, theoretical work is urgently needed to understand the data that suggest a transition in the dominant growth mechanism at galaxy masses of $\sim 10^{9} M_{\odot}$. Are there mechanisms that can prevent gas from reaching the center of galaxies in low-mass galaxies, or is there a barrier to globular cluster accretion in higher mass galaxies?

Observationally, our knowledge of NSCs in lower mass late-type galaxies is woefully lacking. The impressive results from large samples of NSCs in low-mass early types (den Brok et al. 2014; Ordenes-Briceño et al. 2018; Sánchez-Janssen et al. 2019a) clearly highlight the importance for obtaining comparable (i.e., large and homogenously selected) samples also for lower mass late-type galaxies. These data should also help test the environmental dependence of NSC formation. Furthermore, understanding the stellar populations of these NSCs, and determining if they more closely resemble those in low-mass early types or higher mass latetypes would also help us to understand NSC formation more generally. The availability of robust mass measurements (see Fig. 7) is limited primarily to NSCs in higher mass galaxies. More NSC mass measurements at both the high- and lowmass extreme would be of clear interest for understanding NSC scaling relations, enabling us to understand whether they steepen at the high mass end. Also, density measurements in galaxies across the mass spectrum are a key input for understanding tidal disruption event rates, which depend strongly on the NSC density profiles. Finally, we note the lack of data on the resolved kinematics of NSCs (Fig. 11), especially for late-type galaxies, which would provide important insights into the formation history of their NSCs.

Future facilities promise to greatly improve our understanding of NSCs. With the launch of JWST, a better census of NSCs in higher mass galaxies will be possible through high-resolution mid-infrared observations of dusty galaxy cores. Also, the identification of faint AGN enabled by JWST will aid in understanding the frequency of black hole/NSC co-existence in lower mass galaxies (e.g., Dumont et al. 2019). LSST will also play an important role in characterizing the population of black holes in low-mass galaxies through statistics on tidal disruption events, and variability of AGN (e.g., Baldassare et al. 2018). Finally, NSCs will likely be prime 
targets for adaptive optics observations on the 30-m class telescopes (Gullieuszik et al. 2014), enabling the characterization of their internal kinematics, stellar population variations, and central massive black holes.

Acknowledgements Open access funding provided by Projekt DEAL. We would like to thank Iskren Georgiev, Dieu Nguyen, Renuka Pechetti, Ruben Sanchez-Janssen, and Chelsea Spengler for sharing their data, and Nikolay Kacharov for providing Fig. 8. We are grateful to Fabio Antonini, Mark den Brok, Nelson Caldwell, Roberto Capuzzo-Dolcetta, Eric Emsellem, Katja Fahrion, Iskren Georgiev, Oleg Gnedin, Alister Graham, Jenny Greene, Nikolay Kacharov, Alessandra Mastrobuono-Battisti, Mar Mezcua, Thorsten Naab, Mark Norris, Tom Richtler, Hans-Walter Rix, Ruben Sanchez-Janssen, Rainer Schödel, and Monica Valluri for insightful comments. Finally, we would also like to thank the two anonymous referees for constructive reports, and the editors, Andreas Quirrenbach and Frank Schulz, for their valuable comments and editorial assistance. We are grateful for open access funding provided by the Max Planck Society, and support from the University of Utah and its Presidential Scholars program. ACS also acknowledges support from the DAAD Research Stays program, as well as from National Science Foundation Grants AST-1350389 and AST-1813609, and NN gratefully acknowledges support by the Deutsche Forschungsgemeinschaft (DFG, German Research Foundation)_Project-ID 138713538-SFB 881 ("The Milky Way System", subproject B8).

Open Access This article is licensed under a Creative Commons Attribution 4.0 International License, which permits use, sharing, adaptation, distribution and reproduction in any medium or format, as long as you give appropriate credit to the original author(s) and the source, provide a link to the Creative Commons licence, and indicate if changes were made. The images or other third party material in this article are included in the article's Creative Commons licence, unless indicated otherwise in a credit line to the material. If material is not included in the article's Creative Commons licence and your intended use is not permitted by statutory regulation or exceeds the permitted use, you will need to obtain permission directly from the copyright holder. To view a copy of this licence, visit http:// creativecommons.org/licenses/by/4.0/.

\section{References}

Abbott BP, Abbott R, Abbott TD, Abernathy MR, Acernese F, Ackley K, Adams C, Adams T, Addesso P, Adhikari RX et al (2016) Observation of gravitational waves from a binary black hole merger. Phys Rev Lett 116(6):061102. https://doi.org/10.1103/PhysRevLett.116.061102. arXiv:1602.03837

Abbott BP, Abbott R, Abbott TD, Abraham S, Acernese F, Ackley K, Adams C, Adhikari RX, Adya VB, Affeldt C, Agathos M, Agatsuma K, Aggarwal N, Aguiar OD, Aiello L, Ain A, Ajith P, Allen G, Allocca A, Aloy MA, Altin PA, Amato A, Ananyeva A, Anderson SB, Anderson WG, Angelova SV, Scientific Collaboration LIGO, Collaboration Virgo (2019) Binary black hole population properties inferred from the first and second observing runs of advanced LIGO and advanced virgo. ApJ 882(2):L24. https://doi.org/10.3847/2041-8213/ab3800. arXiv:1811.12940

Afanasiev AV, Chilingarian IV, Mieske S, Voggel KT, Picotti A, Hilker M, Seth A, Neumayer N, Frank M, Romanowsky AJ, Hau G, Baumgardt H, Ahn C, Strader J, den Brok M, McDermid R, Spitler L, Brodie J, Walsh JL (2018) A 3.5 million Solar masses black hole in the centre of the ultracompact dwarf galaxy fornax UCD3. MNRAS 477(4):4856-4865. https://doi.org/10.1093/mnras/sty913. arXiv:1804.02938

Agarwal M, Milosavljević M (2011) Nuclear star clusters from clustered star formation. ApJ 729(1):35. https://doi.org/10.1088/0004-637X/729/1/35. arXiv:1008.2986

Aharon D, Perets HB (2015) Formation and evolution of nuclear star clusters with in situ star formation: nuclear cores and age segregation. ApJ 799(2):185. https://doi.org/10.1088/0004-637X/799/2/185. arXiv: 1409.5121 
Aharon D, Perets HB (2016) The impact of mass segregation and star formation on the rates of gravitational-wave sources from extreme mass ratio inspirals. ApJ 830(1):L1. https://doi.org/10. 3847/2041-8205/830/1/L1. arXiv:1609.01715

Aharon D, Mastrobuono Battisti A, Perets HB (2016) The history of tidal disruption events in galactic nuclei. ApJ 823:137. https://doi.org/10.3847/0004-637X/823/2/137. arXiv:1507.08287

Ahn CP, Seth AC, den Brok M, Strader J, Baumgardt H, van den Bosch R, Chilingarian I, Frank M, Hilker M, McDermid R, Mieske S, Romanowsky AJ, Spitler L, Brodie J, Neumayer N, Walsh JL (2017) Detection of supermassive black holes in two virgo ultracompact dwarf galaxies. ApJ 839(2):72. https://doi.org/10.3847/1538-4357/aa6972. arXiv:1703.09221

Ahn CP, Seth AC, Cappellari M, Krajnović D, Strader J, Voggel KT, Walsh JL, Bahramian A, Baumgardt H, Brodie J, Chilingarian I, Chomiuk L, den Brok M, Frank M, Hilker M, McDermid RM, Mieske S, Neumayer N, Nguyen DD, Pechetti R, Romanowsky AJ, Spitler L (2018) The black hole in the most massive ultracompact dwarf galaxy M59-UCD3. ApJ 858(2):102. https://doi.org/10.3847/15384357/aabc57. arXiv:1804.02399

Alfaro-Cuello M, Kacharov N, Neumayer N, Bianchini P, Mastrobuono-Battisti A, Lützgendorf N, Seth AC, Böker T, Kamann S, Leaman R, Watkins LL, van de Ven G (2020) A deep view into the nucleus of the sagittarius dwarf spheroidal galaxy with MUSE. II. Kinematic characterization of the stellar populations. ApJ 892(1):20. https://doi.org/10.3847/1538-4357/ab77bb. arXiv:2002.07814

Alfaro-Cuello M, Kacharov N, Neumayer N, Luetzgendorf N, Seth AC, Boeker T, Kamann S, Leaman R, van de Ven G, Bianchini P, Watkins LL, Lyubenova M (2019) A deep view into the nucleus of the Sagittarius Dwarf Spheroidal Galaxy with MUSE. I. Data and stellar population characterization. ApJ 886(1):57. https://doi.org/10.3847/1538-4357/ab1b2c. arXiv:1909.10529

Allen DA, Hyland AR, Hillier DJ (1990) The source of luminosity at the Galactic Centre. MNRAS 244:706

Amaro-Seoane P, Chen X (2014) The fragmenting past of the disk at the galactic center: the culprit for the missing red giants. ApJ 781(1):L18. https://doi.org/10.1088/2041-8205/781/1/L18. arXiv:1310.0458

Amaro-Seoane P, Audley H, Babak S, Baker J, Barausse E, Bender P, Berti E, Binetruy P, Born M (2017) Laser interferometer space antenna. arXiv:1702.00786

Amaro-Seoane P, Chen X, Schödel R, Casanellas J (2020) Making bright giants invisible at the galactic centre. MNRAS 492:250-255. https://doi.org/10.1093/mnras/stz3507. arXiv:1910.04774

Antonini F (2013) Origin and growth of nuclear star clusters around massive black holes. ApJ 763(1):62. https://doi.org/10.1088/0004-637X/763/1/62. arXiv:1207.6589

Antonini F (2014) On the distribution of stellar remnants around massive black holes: slow mass segregation, star cluster inspirals, and correlated orbits. ApJ 794(2):106. https://doi.org/10.1088/ 0004-637X/794/2/106. arXiv:1402.4865

Antonini F, Perets HB (2012) Secular evolution of compact binaries near massive black holes: gravitational wave sources and other exotica. ApJ 757(1):27. https://doi.org/10.1088/0004-637X/ 757/1/27. arXiv: 1203.2938

Antonini F, Rasio FA (2016) Merging black hole binaries in galactic nuclei: implications for advancedLIGO detections. ApJ 831:187. https://doi.org/10.3847/0004-637X/831/2/187. arXiv:1606.04889

Antonini F, Capuzzo-Dolcetta R, Mastrobuono-Battisti A, Merritt D (2012) Dissipationless formation and evolution of the milky way nuclear star cluster. ApJ 750(2):111. https://doi.org/10.1088/0004-637X/ 750/2/111. arXiv:1110.5937

Antonini F, Barausse E, Silk J (2015) The coevolution of nuclear star clusters, massive black holes, and their host galaxies. ApJ 812:72. https://doi.org/10.1088/0004-637X/812/1/72. arXiv:1506.02050

Antonini F, Gieles M, Gualandris A (2019) Black hole growth through hierarchical black hole mergers in dense star clusters: implications for gravitational wave detections. MNRAS 486(4):5008-5021. https://doi.org/10.1093/mnras/stz1149. arXiv:1811.03640

Arca-Sedda M, Capuzzo-Dolcetta R (2017) Lack of nuclear clusters in dwarf spheroidal galaxies: implications for massive black holes formation and the cusp/core problem. MNRAS 464(3):3060-3070. https://doi.org/10.1093/mnras/stw2483. arXiv:1611.01088

Arca-Sedda M, Capuzzo-Dolcetta R, Antonini F, Seth A (2015) Henize 2-10: the ongoing formation of a nuclear star cluster around a massive black hole. ApJ 806(2):220. https://doi.org/10.1088/0004637X/806/2/220. arXiv:1501.04567

Askar A, Arca Sedda M, Giersz M (2018) MOCCA-SURVEY database I: galactic globular clusters harbouring a black hole subsystem. MNRAS 478(2):1844-1854. https://doi.org/10.1093/mnras/ sty1186. arXiv:1802.05284 
Auchettl K, Guillochon J, Ramirez-Ruiz E (2017) New physical insights about tidal disruption events from a comprehensive observational inventory at X-ray wavelengths. ApJ 838(2):149. https://doi. org/10.3847/1538-4357/aa633b. arXiv:1611.02291

Bacon R, Emsellem E, Monnet G, Nieto JL (1994) Sub-arcsecond 2D photometry and spectroscopy of the nucleus of M 31: the supermassive black hole revisited. A\&A 281:691-717 arXiv:astro-ph/9309008

Bacon R, Emsellem E, Combes F, Copin Y, Monnet G, Martin P (2001) The M 31 double nucleus probed with OASIS. A natural $m=1$ mode? A\&A 371:409-428. https://doi.org/10.1051/0004-6361: 20010317. arXiv:astro-ph/0010567

Bahcall JN, Wolf RA (1976) Star distribution around a massive black hole in a globular cluster. ApJ 209:214-232. https://doi.org/10.1086/154711

Bailey ME (1980) On the fate of stellar mass loss in galactic nuclei. MNRAS 191:195-206. https://doi. org/10.1093/mnras/191.2.195

Balcells M, Graham AW, Domínguez-Palmero L, Peletier RF (2003) Galactic bulges from Hubble Space Telescope near-infrared camera multi-object spectrometer observations: the lack of $\mathrm{r}^{1 / 4}$ bulges. ApJ 582(2):L79-L82. https://doi.org/10.1086/367783. arXiv:astro-ph/0212184

Balcells M, Graham AW, Peletier RF (2007) Galactic bulges from Hubble Space Telescope NICMOS observations: central galaxian objects, and nuclear profile slopes. ApJ 665:1084-1103. https://doi. org/10.1086/519752. arXiv:arXiv:astro-ph/0404379

Baldassare VF, Gallo E, Miller BP, Plotkin RM, Treu T, Valluri M, Woo JH (2014) AMUSE-field. II. Nucleation of early-type galaxies in the field versus cluster environment. ApJ 791(2):133. https:// doi.org/10.1088/0004-637X/791/2/133. arXiv:1406.6697

Baldassare VF, Reines AE, Gallo E, Greene JE (2015) A 50,000M $\odot$ solar mass black hole in the nucleus of RGG 118. ApJ 809(1):L14. https://doi.org/10.1088/2041-8205/809/1/L14. arXiv:1506. 07531

Baldassare VF, Geha M, Greene J (2018) Identifying AGNs in low-mass galaxies via long-term optical variability. ApJ 868(2):152. https://doi.org/10.3847/1538-4357/aae6cf. arXiv:1808.09578

Balick B, Brown RL (1974) Intense sub-arcsecond structure in the galactic center. ApJ 194:265-270. https://doi.org/10.1086/153242

Barth AJ, Strigari LE, Bentz MC, Greene JE, Ho LC (2009) Dynamical constraints on the masses of the nuclear star cluster and black hole in the late-type spiral galaxy NGC 3621. ApJ 690(1):1031-1044. https://doi.org/10.1088/0004-637X/690/1/1031. arXiv:0809.1066

Bartko H, Martins F, Trippe S, Fritz TK, Genzel R, Ott T, Eisenhauer F, Gillessen S, Paumard T, Alexander T (2010) An extremely top-heavy initial mass function in the galactic center stellar disks. ApJ 708(1):834-840. https://doi.org/10.1088/0004-637X/708/1/834. arXiv:0908.2177

Bassino LP, Muzzio JC, Rabolli M (1994) Are globular clusters the nuclei of cannibalized dwarf galaxies? ApJ 431:634. https://doi.org/10.1086/174514

Baumgardt H (2017) N-body modelling of globular clusters: masses, mass-to-light ratios and intermediate-mass black holes. MNRAS 464(2):2174-2202. https://doi.org/10.1093/mnras/ stw2488. arXiv:1609.08794

Baumgardt H, Amaro-Seoane P, Schödel R (2018) The distribution of stars around the Milky Way's central black hole. III. Comparison with simulations. A\&A 609:A28. https://doi.org/10.1051/00046361/201730462. arXiv:1701.03818

Baumgardt H, He C, Sweet SM, Drinkwater M, Sollima A, Hurley J, Usher C, Kamann S, Dalgleish H, Dreizler S, Husser TO (2019) No evidence for intermediate-mass black holes in the globular clusters $\omega$ Cen and NGC 6624. MNRAS 488(4):5340-5351. https://doi.org/10.1093/mnras/stz2060. arXiv: 1907.10845

Becklin EE, Neugebauer G (1968) Infrared observations of the galactic center. ApJ 151:145. https://doi. org/10.1086/149425

Becklin EE, Neugebauer G (1975) High-resolution maps of the galactic center at 2.2 and 10 microns. ApJ 200:L71-L74. https://doi.org/10.1086/181899

Begelman MC, Rees MJ (1978) The fate of dense stellar systems. MNRAS 185:847-860. https://doi.org/ $10.1093 / \mathrm{mnras} / 185.4 .847$

Bekki K (2007) The formation of stellar galactic nuclei through dissipative gas dynamics. PASA 24(2):77-94. https://doi.org/10.1071/AS07008

Bekki K, Freeman KC (2003) Formation of $\omega$ Centauri from an ancient nucleated dwarf galaxy in the young Galactic disc. MNRAS 346(2):L11-L15. https://doi.org/10.1046/j.1365-2966.2003.07275.x. arXiv:astro-ph/0310348 
Bekki K, Graham AW (2010) On the transition from nuclear-cluster-to black-hole-dominated galaxy cores. ApJ 714(2):L313-L317. https://doi.org/10.1088/2041-8205/714/2/L313. arXiv:1004.3627

Bekki K, Couch WJ, Drinkwater MJ, Shioya Y (2003) Galaxy threshing and the origin of ultra-compact dwarf galaxies in the Fornax cluster. MNRAS 344(2):399-411. https://doi.org/10.1046/j.1365-8711. 2003.06916.x. arXiv:astro-ph/0308243

Bekki K, Couch WJ, Shioya Y (2006) Dissipative transformation of nonnucleated dwarf galaxies into nucleated systems. ApJ 642(2):L133-L136. https://doi.org/10.1086/504588. arXiv:astro-ph/ 0604340

Bender R, Kormendy J, Bower G, Green R, Thomas J, Danks AC, Gull T, Hutchings JB, Joseph CL, Kaiser ME, Lauer TR, Nelson CH, Richstone D, Weistrop D, Woodgate B (2005) HST STIS spectroscopy of the triple nucleus of M31: two nested disks in Keplerian rotation around a supermassive black hole. ApJ 631(1):280-300. https://doi.org/10.1086/432434. arXiv:astro-ph/ 0509839

Bianchini P, Varri AL, Bertin G, Zocchi A (2013) Rotating globular clusters. ApJ 772(1):67. https://doi. org/10.1088/0004-637X/772/1/67. arXiv:1305.6025

Binggeli B, Sandage A, Tammann GA (1985) Studies of the Virgo cluster. II-A catalog of 2096 galaxies in the virgo cluster area. AJ 90:1681-1759. https://doi.org/10.1086/113874

Birchall KL, Watson MG, Aird J (2020) X-ray detected AGN in SDSS dwarf galaxies. MNRAS 492(2):2268-2284. https://doi.org/10.1093/mnras/staa040. arXiv:2001.03135

Bland-Hawthorn J, Gerhard O (2016) The galaxy in context: structural, kinematic, and integrated properties. ARA\&A 54:529-596. https://doi.org/10.1146/annurev-astro-081915-023441. arXiv: 1602.07702

Bland-Hawthorn J, Vlajić M, Freeman KC, Draine BT (2005) NGC 300: an extremely faint, outer stellar disk observed to 10 scale lengths. Astrophys J 629(1):239-249. https://doi.org/10.1086/430512. arXiv:astro-ph/0503488

Blanton MR, Lupton RH, Schlegel DJ, Strauss MA, Brinkmann J, Fukugita M, Loveday J (2005) The properties and luminosity function of extremely low luminosity galaxies. ApJ 631(1):208-230. https://doi.org/10.1086/431416. arXiv:astro-ph/0410164

Blum RD, Ramírez SV, Sellgren K, Olsen K (2003) Really cool stars and the star formation history at the galactic center. ApJ 597(1):323-346. https://doi.org/10.1086/378380. arXiv:astro-ph/0307291

Böker T, Förster-Schreiber NM, Genzel R (1997) Near-infrared imaging spectroscopy of IC 342: evolution of a bar-driven central starburst. AJ 114:1883. https://doi.org/10.1086/118612

Böker T, van der Marel RP, Vacca WD (1999) CO band head spectroscopy of IC 342: mass and age of the nuclear star cluster. AJ 118(2):831-842. https://doi.org/10.1086/300985. arXiv:astro-ph/9903457

Böker T, van der Marel RP, Mazzuca L, Rix HW, Rudnick G, Ho LC, Shields JC (2001) A young stellar cluster in the nucleus of NGC 4449. AJ 121(3):1473-1481. https://doi.org/10.1086/319415. arXiv: astro-ph/0010542

Böker T, Laine S, van der Marel RP, Sarzi M, Rix HW, Ho LC, Shields JC (2002) A Hubble Space Telescope census of nuclear star clusters in late-type spiral galaxies. I. Observations and image analysis. AJ 123:1389-1410. https://doi.org/10.1086/339025

Böker T, Sarzi M, McLaughlin DE, van der Marel RP, Rix HW, Ho LC, Shields JC (2004) A Hubble Space Telescope census of nuclear star clusters in late-type spiral galaxies. II. Cluster sizes and structural parameter correlations. AJ 127:105-118. https://doi.org/10.1086/380231. arXiv:astro-ph/ 0309761

Boldrini P, Mohayaee R, Silk J (2019) Fornax globular cluster distributions: implications for the cuspcore problem. MNRAS 485(2):2546-2557. https://doi.org/10.1093/mnras/stz573. arXiv:1903.00354

Bothun GD, Mould JR (1988) Medium-resolution spectroscopy of nucleated dwarf elliptical galaxies. ApJ 324:123. https://doi.org/10.1086/165885

Bothun GD, Mould JR, Wirth A, Caldwell N (1985) An investigation of dwarf galaxies in the Virgo cluster. AJ 90:697-707. https://doi.org/10.1086/113778

Bower GA, Green RF, Danks A, Gull T, Heap S, Hutchings J, Joseph C, Kaiser ME, Kimble R, Kraemer S, Weistrop D, Woodgate B, Lindler D, Hill RS, Malumuth EM, Baum S, Sarajedini V, Heckman TM, Wilson AS, Richstone DO (1998) Kinematics of the nuclear ionized gas in the radio galaxy M84 (NGC 4374). ApJ 492(2):L111-L114. https://doi.org/10.1086/311109. arXiv:astro-ph/9710264

Bower GA, Green RF, Bender R, Gebhardt K, Lauer TR, Magorrian J, Richstone DO, Danks A, Gull T, Hutchings J, Joseph C, Kaiser ME, Weistrop D, Woodgate B, Nelson C, Malumuth EM (2001) Evidence of a supermassive black hole in the galaxy NGC 1023 from the nuclear stellar dynamics. ApJ 550(1):75-86. https://doi.org/10.1086/319730. arXiv:astro-ph/0011204 
Brown G, Gnedin OY, Li H (2018) Nuclear star clusters in cosmological simulations. ApJ 864(1):94. https://doi.org/10.3847/1538-4357/aad595. arXiv:1804.09819

Bruzual G, Charlot S (2003) Stellar population synthesis at the resolution of 2003. MNRAS 344(4):1000-1028. https://doi.org/10.1046/j.1365-8711.2003.06897.x. arXiv:astro-ph/0309134

Bub MW, Petrovich C (2020) Compact-object mergers in the galactic center: evolution in triaxial clusters. ApJ 894:15. https://doi.org/10.3847/1538-4357/ab8461. arXiv:1910.02079

Buchholz RM, Schödel R, Eckart A (2009) Composition of the galactic center star cluster. Population analysis from adaptive optics narrow band spectral energy distributions. A\&A 499(2):483-501. https://doi.org/10.1051/0004-6361/200811497. arXiv:0903.2135

Bullock JS, Boylan-Kolchin M (2017) Small-scale challenges to the $\Lambda$ CDM paradigm. ARA\&A 55(1):343-387. https://doi.org/10.1146/annurev-astro-091916-055313. arXiv:1707.04256

Caldwell N (1983) Structure and stellar content of dwarf elliptical galaxies. AJ 88:804-812. https://doi. org/10.1086/113367

Caldwell N, Bothun GD (1987) Dwarf elliptical galaxies in the fornax cluster. II. Their structure and stellar populations. AJ 94:1126. https://doi.org/10.1086/114550

Caldwell N, Strader J, Sand DJ, Willman B, Seth AC (2017) The faint globular cluster in the dwarf galaxy Andromeda I. PASA 34:e039. https://doi.org/10.1017/pasa.2017.35. arXiv:1708.03596

Cappellari M (2016) Structure and kinematics of early-type galaxies from integral field spectroscopy. ARA\&A 54:597-665. https://doi.org/10.1146/annurev-astro-082214-122432. arXiv:1602.04267

Cappellari M, McDermid RM, Alatalo K, Blitz L, Bois M, Bournaud F, Bureau M, Crocker AF, Davies RL, Davis TA, de Zeeuw PT, Duc PA, Emsellem E, Khochfar S, Krajnović D, Kuntschner H, Morganti R, Naab T, Oosterloo T, Sarzi M, Scott N, Serra P, Weijmans AM, Young LM (2013) The $\mathrm{ATLAS}^{3 D}$ project-XX. Mass-size and mass- distributions of early-type galaxies: bulge fraction drives kinematics, mass-to-light ratio, molecular gas fraction and stellar initial mass function. MNRAS 432(3):1862-1893. https://doi.org/10.1093/mnras/stt644. arXiv:1208.3523

Capuzzo-Dolcetta R (1993) The evolution of the globular cluster system in a triaxial galaxy: can a galactic nucleus form by globular cluster capture? ApJ 415:616. https://doi.org/10.1086/173189. arXiv:astro-ph/9301006

Capuzzo-Dolcetta R, Mastrobuono-Battisti A (2009) Globular cluster system erosion in elliptical galaxies. A\&A 507(1):183-193. https://doi.org/10.1051/0004-6361/200912255. arXiv:0904.0526

Capuzzo-Dolcetta R, Miocchi P (2008a) Merging of globular clusters in inner galactic regions. II. Nuclear star cluster formation. ApJ 681(2):1136-1147. https://doi.org/10.1086/588017. arXiv:0801.1072

Capuzzo-Dolcetta R, Miocchi P (2008b) Self-consistent simulations of nuclear cluster formation through globular cluster orbital decay and merging. MNRAS 388(1):L69-L73. https://doi.org/10.1111/j. 1745-3933.2008.00501.x. arXiv:0804.4421

Capuzzo-Dolcetta R, Tostae Melo I (2017) On the relation between the mass of compact massive objects and their host galaxies. MNRAS 472(4):4013-4023. https://doi.org/10.1093/mnras/stx2246. arXiv: 1708.08391

Carlsten SG, Greco JP, Beaton RL, Greene JE (2019) Wide-field survey of dwarf satellite systems around 10 hosts in the local volume. ApJ 891(2):144. https://doi.org/10.3847/1538-4357/ab7758. arXiv: 1909.07389

Carollo CM, Stiavelli M (1998) Spiral galaxies with WFPC2. III. Nuclear cusp slopes. AJ 115:2306-2319. https://doi.org/10.1086/300373. arXiv:astro-ph/9804010

Carollo CM, Stiavelli M, de Zeeuw PT, Mack J (1997) Spiral galaxies with WFPC2. I. Nuclear morphology, bulges, star clusters, and surface brightness profiles. AJ 114:2366. https://doi.org/10. $1086 / 118654$

Carollo CM, Stiavelli M, Mack J (1998) Spiral galaxies with WFPC2. II. The nuclear properties of 40 objects. AJ 116:68-84. https://doi.org/10.1086/300407. arXiv:astro-ph/9804007

Carollo CM, Stiavelli M, Seigar M, de Zeeuw PT, Dejonghe H (2002) Spiral galaxies with HST/ NICMOS. I. Nuclear morphologies, color maps, and distinct nuclei. AJ 123:159-183. https://doi. org/10.1086/324725. arXiv:astro-ph/0110281

Carson DJ, Barth AJ, Seth AC, den Brok M, Cappellari M, Greene JE, Ho LC, Neumayer N (2015) The structure of nuclear star clusters in nearby late-type spiral galaxies from Hubble Space Telescope wide field camera 3 imaging. AJ 149:170. https://doi.org/10.1088/0004-6256/149/5/170. arXiv: 1501.05586

Cen R (2001) Synchronized formation of subgalactic systems at cosmological reionization: origin of halo globular clusters. ApJ 560(2):592-598. https://doi.org/10.1086/323071. arXiv:astro-ph/0101197 
Chabrier G (2003) Galactic stellar and substellar initial mass function. PASP 115(809):763-795. https:// doi.org/10.1086/376392. arXiv:astro-ph/0304382

Chang P, Murray-Clay R, Chiang E, Quataert E (2007) The origin of the young stars in the nucleus of M31. ApJ 668(1):236-244. https://doi.org/10.1086/521018. arXiv:0704.3831

Chatzopoulos S, Fritz TK, Gerhard O, Gillessen S, Wegg C, Genzel R, Pfuhl O (2015) The old nuclear star cluster in the Milky Way: dynamics, mass, statistical parallax, and black hole mass. MNRAS 447(1):948-968. https://doi.org/10.1093/mnras/stu2452. arXiv:1403.5266

Chilingarian IV (2009) Evolution of dwarf early-type galaxies - I. Spatially resolved stellar populations and internal kinematics of Virgo cluster dE/dS0 galaxies. MNRAS 394(3):1229-1248. https://doi. org/10.1111/j.1365-2966.2009.14450.x. arXiv:0812.3272

Chilingarian IV, Mamon GA (2008) SDSSJ124155.33+114003.7—a missing link between compact elliptical and ultracompact dwarf galaxies. MNRAS 385(1):L83-L87. https://doi.org/10.1111/j. 1745-3933.2008.00438.x

Chilingarian IV, Katkov IY, Zolotukhin IY, Grishin KA, Beletsky Y, Boutsia K, Osip DJ (2018) A population of bona fide intermediate-mass black holes identified as low-luminosity active galactic nuclei. ApJ 863(1):1. https://doi.org/10.3847/1538-4357/aad184. arXiv:1805.01467

Choksi N, Volonteri M, Colpi M, Gnedin OY, Li H (2019) The star clusters that make black hole binaries across cosmic time. ApJ 873(1):100. https://doi.org/10.3847/1538-4357/aaffde. arXiv:1809.01164

Gravity Collaboration, Abuter R, Amorim A, Anugu N, Bauböck M, Benisty M, Berger JP, Blind N, Bonnet H, Brandner W, Buron A, Collin C, Chapron F, Clénet Y, Coude Du Foresto V, de Zeeuw PT, Deen C, Delplancke-Ströbele F, Dembet R, Dexter J, Duvert G, Eckart A, Eisenhauer F, Finger G, Förster Schreiber NM, Fédou P, Garcia P, Garcia Lopez R, Gao F, Gendron E, Genzel R, Gillessen S, Gordo P, Habibi M, Haubois X, Haug M, Haußmann F, Henning T, Hippler S, Horrobin M, Hubert Z, Hubin N, Jimenez Rosales A, Jochum L, Jocou K, Kaufer A, Kellner S, Kendrew S, Kervella P, Kok Y, Kulas M, Lacour S, Lapeyrère V, Lazareff B, Le Bouquin JB, Léna P, Lippa M, Lenzen R, Mérand A, Müler E, Neumann U, Ott T, Palanca L, Paumard T, Pasquini L, Perraut K, Perrin G, Pfuhl O, Plewa PM, Rabien S, Ramírez A, Ramos J, Rau C, Rodríguez-Coira G, Rohloff RR, Rousset G, Sanchez-Bermudez J, Scheithauer S, Schöller M, Schuler N, Spyromilio J, Straub O, Straubmeier C, Sturm E, Tacconi LJ, Tristram KRW, Vincent F, von Fellenberg S, Wank I, Waisberg I, Widmann F, Wieprecht E, Wiest M, Wiezorrek E, Woillez J, Yazici S, Ziegler D, Zins G (2018) Detection of the gravitational redshift in the orbit of the star S2 near the Galactic centre massive black hole. A\&A 615:L15. https://doi.org/10.1051/0004-6361/201833718. arXiv:1807. 09409

Gravity Collaboration, Abuter R, Amorim A, Bauböck M, Berger JP, Bonnet H, Brandner W, Coudé Du Clénet Y, Foresto V, de Zeeuw PT (2019) A geometric distance measurement to the Galactic center black hole with 0.3\% uncertainty. A\&A 625:L10. https://doi.org/10.1051/0004-6361/201935656

Conroy C (2013) Modeling the panchromatic spectral energy distributions of galaxies. ARA\&A 51(1):393-455. https://doi.org/10.1146/annurev-astro-082812-141017. arXiv:1301.7095

Côté P, Piatek S, Ferrarese L, Jordán A, Merritt D, Peng EW, Haşegan M, Blakeslee JP, Mei S, West MJ, Milosavljević M, Tonry JL (2006) The ACS Virgo cluster survey. VIII. The nuclei of early-type galaxies. ApJS 165:57-94. https://doi.org/10.1086/504042

Côté P, Ferrarese L, Jordán A, Blakeslee JP, Chen CW, Infante L, Merritt D, Mei S, Peng EW, Tonry JL, West AA, West MJ (2007) The ACS fornax cluster survey. II. The central brightness profiles of early-type galaxies: a characteristic radius on nuclear scales and the transition from central luminosity deficit to excess. ApJ 671:1456-1465. https://doi.org/10.1086/522822. arXiv:0711.1358

Cretton N, van den Bosch FC (1999) Evidence for a massive black hole in the S0 galaxy NGC 4342. ApJ 514(2):704-724. https://doi.org/10.1086/306971. arXiv:astro-ph/9805324

Crnojević D, Sand DJ, Zaritsky D, Spekkens K, Willman B, Hargis JR (2016) Deep imaging of eridanus II and its lone star cluster. ApJ 824(1):L14. https://doi.org/10.3847/2041-8205/824/1/L14. arXiv: 1604.08590

Da Costa GS (2016) Are the globular clusters with significant internal [Fe/H] spreads all former dwarf galaxy nuclei? In: Bragaglia A, Arnaboldi M, Rejkuba M, Romano D (eds) The general assembly of galaxy halos: structure, origin and evolution, IAU Symposium, vol 317, pp 110-115. https://doi.org/ 10.1017/S174392131500678X. arXiv:1510.00873

Dale JE, Davies MB, Church RP, Freitag M (2009) Red giant stellar collisions in the Galactic Centre. MNRAS 393:1016-1033. https://doi.org/10.1111/j.1365-2966.2008.14254.x. arXiv:0811.3111

Davies R, Kasper M (2012) Adaptive optics for astronomy. Annu Rev Astron Astrophys 50:305-351. https://doi.org/10.1146/annurev-astro-081811-125447. arXiv:1201.5741 
Davies MB, Blackwell R, Bailey VC, Sigurdsson S (1998) The destructive effects of binary encounters on red giants in the Galactic Centre. MNRAS 301(3):745-753. https://doi.org/10.1046/j.1365-8711. 1998.02027.x

Davies MB, Miller MC, Bellovary JM (2011) Supermassive black hole formation via gas accretion in nuclear stellar clusters. ApJ 740(2):L42. https://doi.org/10.1088/2041-8205/740/2/L42. arXiv:1106. 5943

Davis et al T (2020) Revealing the intermediate-mass black hole at the heart of the dwarfgalaxy NGC 404 with sub-parsec resolution ALMA observations. MNRAS

de Francesco G, Capetti A, Marconi A (2006) Measuring supermassive black holes with gas kinematics: the active S0 galaxy <ASTROBJ > NGC 3998</ASTROBJ > A\&A 460(2):439-448. https:// doi.org/10.1051/0004-6361:20065826. arXiv:astro-ph/0609603

De Lorenzi F, Hartmann M, Debattista VP, Seth AC, Gerhard O (2013) Three-integral multicomponent dynamical models and simulations of the nuclear star cluster in NGC 4244. MNRAS 429:2974-2985. https://doi.org/10.1093/mnras/sts545. arXiv:1208.2161

Demarque P, Virani S (2007) The hot stars in orbit around the M 31 central supermassive black hole: are they young or old? A\&A 461(2):651-656. https://doi.org/10.1051/0004-6361:20065921. arXiv: astro-ph/0603326

den Brok M, Peletier RF, Seth A, Balcells M, Dominguez L, Graham AW, Carter D, Erwin P, Ferguson HC, Goudfrooij P, Guzmán R, Hoyos C, Jogee S, Lucey J, Phillipps S, Puzia T, Valentijn E, Kleijn GV, Weinzirl T (2014) The HST/ACS coma cluster survey-X. Nuclear star clusters in low-mass early-type galaxies: scaling relations. MNRAS 445:2385-2403. https://doi.org/10.1093/mnras/ stu1906. arXiv: 1409.4766

den Brok M, Seth AC, Barth AJ, Carson DJ, Neumayer N, Cappellari M, Debattista VP, Ho LC, Hood CE, McDermid RM (2015) Measuring the mass of the central black hole in the bulgeless galaxy NGC 4395 from gas dynamical modeling. ApJ 809(1):101. https://doi.org/10.1088/0004-637X/809/ $1 / 101$

Devereux N, Ford H, Tsvetanov Z, Jacoby G (2003) STIS spectroscopy of the central 10 parsecs of M81: evidence for a massive black hole. AJ 125(3):1226-1235. https://doi.org/10.1086/367595

Do T, Ghez AM, Morris MR, Lu JR, Matthews K, Yelda S, Larkin J (2009) High angular resolution integral-field spectroscopy of the galaxy's nuclear cluster: a missing stellar cusp? ApJ 703(2):1323-1337. https://doi.org/10.1088/0004-637X/703/2/1323. arXiv:0908.0311

Do T, Kerzendorf W, Winsor N, Støstad M, Morris MR, Lu JR, Ghez AM (2015) Discovery of lowmetallicity stars in the central parsec of the milky way. ApJ 809(2):143. https://doi.org/10.1088/ 0004-637X/809/2/143. arXiv:1506.07891

Do T, Hees A, Ghez A, Martinez GD, Chu DS, Jia S, Sakai S, Lu JR, Gautam AK, O’Neil KK, Becklin EE, Morris MR, Matthews K, Nishiyama S, Campbell R, Chappell S, Chen Z, Ciurlo A, Dehghanfar A, Gallego-Cano E, Kerzendorf WE, Lyke JE, Naoz S, Saida H, Schödel R, Takahashi M, Takamori Y, Witzel G, Wizinowich P (2019) Relativistic redshift of the star S0-2 orbiting the Galactic Center supermassive black hole. Science 365(6454):664-668. https://doi.org/10.1126/science.aav8137. arXiv:1907.10731

Dressler A, Richstone DO (1988) Stellar dynamics in the nuclei of M31 and M32: evidence for massive black holes. ApJ 324:701. https://doi.org/10.1086/165930

Drinkwater MJ, Jones JB, Gregg MD, Phillipps S (2000) Compact stellar systems in the fornax cluster: super-massive star clusters or extremely compact dwarf galaxies? PASA 17(3):227-233. https://doi. org/10.1071/AS00034. arXiv:astro-ph/0002003

Dumont A, Seth A, Strader J, Greene JE, Burtscher L, Neumayer N (2019) Surprisingly strong K-band emission found in low-luminosity active galactic nuclei. ApJ 888(1):19. https://doi.org/10.3847/ 1538-4357/ab5798. arXiv:1909.07323

Ebisuzaki T, Makino J, Tsuru TG, Funato Y, Portegies Zwart S, Hut P, McMillan S, Matsushita S, Matsumoto H, Kawabe R (2001) Missing link found? The "runaway" path to supermassive black holes. ApJ 562(1):L19-L22. https://doi.org/10.1086/338118. arXiv:astro-ph/0106252

Eigenthaler P, Puzia TH, Taylor MA, Ordenes-Briceño Y, Muñoz RP, Ribbeck KX, Alamo-Martínez KA, Zhang H, Ángel S, Capaccioli M (2018) The Next Generation Fornax Survey (NGFS). II. The central dwarf galaxy population. ApJ 855(2):142. https://doi.org/10.3847/1538-4357/aaab60. arXiv: 1801.02633

Emami R, Loeb A (2019) Gravitational waves from stellar mass black holes around SgrA*. arXiv:1903. 02579 (arXiv e-prints) 
Emsellem E, van de Ven G (2008) Formation of central massive objects via tidal compression. ApJ 674(2):653-659. https://doi.org/10.1086/524720. arXiv:0710.3161

Emsellem E, Dejonghe H, Bacon R (1999) Dynamical models of NGC 3115. MNRAS 303(3):495-514. https://doi.org/10.1046/j.1365-8711.1999.02210.x. arXiv:astro-ph/9810306

Emsellem E, Renaud F, Bournaud F, Elmegreen B, Combes F, Gabor JM (2015) The interplay between a galactic bar and a supermassive black hole: nuclear fuelling in a subparsec resolution galaxy simulation. MNRAS 446(3):2468-2482. https://doi.org/10.1093/mnras/stu2209. arXiv:1410.6479

Erwin P, Gadotti DA (2012) Do nuclear star clusters and supermassive black holes follow the same hostgalaxy correlations? Adv Astron 2012:946368. https://doi.org/10.1155/2012/946368. arXiv:1112. 2740

Evstigneeva EA, Drinkwater MJ, Peng CY, Hilker M, De Propris R, Jones JB, Phillipps S, Gregg MD, Karick AM (2008) Structural properties of ultra-compact dwarf galaxies in the fornax and virgo clusters. AJ 136(1):461-478. https://doi.org/10.1088/0004-6256/136/1/461. arXiv:0804.4353

Fahrion K, Lyubenova M, van de Ven G, Leaman R, Hilker M, Martín-Navarro I, Zhu L, Alfaro-Cuello M, Coccato L, Corsini EM, Falcón-Barroso J, Iodice E, McDermid RM, Sarzi M, de Zeeuw T (2019) Constraining nuclear star cluster formation using MUSE-AO observations of the early-type galaxy FCC 47. A\&A 628:A92. https://doi.org/10.1051/0004-6361/201935832. arXiv:1907.01007

Feldmeier A, Neumayer N, Seth A, Schödel R, Lützgendorf N, de Zeeuw PT, Kissler-Patig M, Nishiyama S, Walcher CJ (2014) Large scale kinematics and dynamical modelling of the Milky Way nuclear star cluster. A\&A 570:A2. https://doi.org/10.1051/0004-6361/201423777. arXiv:1406.2849

Feldmeier-Krause A, Neumayer N, Schödel R, Seth A, Hilker M, de Zeeuw PT, Kuntschner H, Walcher CJ, Lützgendorf N, Kissler-Patig M (2015) KMOS view of the Galactic centre. I. Young stars are centrally concentrated. A\&A 584:A2. https://doi.org/10.1051/0004-6361/201526336. arXiv:1509. 04707

Feldmeier-Krause A, Kerzendorf W, Neumayer N, Schödel R, Nogueras-Lara F, Do T, de Zeeuw PT, Kuntschner H (2017a) KMOS view of the Galactic Centre - II. Metallicity distribution of late-type stars. MNRAS 464(1):194-209. https://doi.org/10.1093/mnras/stw2339. arXiv:1610.01623

Feldmeier-Krause A, Zhu L, Neumayer N, van de Ven G, de Zeeuw PT, Schödel R (2017b) Triaxial orbitbased modelling of the milky way nuclear star cluster. MNRAS 466(4):4040-4052. https://doi.org/ 10.1093/mnras/stw3377. arXiv:1701.01583

Ferguson HC, Sandage A (1989) The spatial distributions and intrinsic shapes of dwarf elliptical galaxies in the virgo and fornax clusters. ApJ 346:L53. https://doi.org/10.1086/185577

Ferrarese L, Ford HC, Jaffe W (1996) Evidence for a massive black hole in the active galaxy NGC 4261 from Hubble Space Telescope images and spectra. ApJ 470:444. https://doi.org/10.1086/177876

Ferrarese L, Côté P, Dalla Bontà E, Peng EW, Merritt D, Jordán A, Blakeslee JP, Haşegan M, Mei S, Piatek S, Tonry JL, West MJ (2006a) A fundamental relation between compact stellar nuclei, supermassive black holes, and their host galaxies. ApJ 644:L21-L24. https://doi.org/10.1086/ 505388. arXiv:astro-ph/0603840

Ferrarese L, Côté P, Jordán A, Peng EW, Blakeslee JP, Piatek S, Mei S, Merritt D, Milosavljević M, Tonry JL (2006b) The ACS Virgo cluster survey. VI. Isophotal analysis and the structure of earlytype galaxies. ApJS 164(2):334-434. https://doi.org/10.1086/501350. arXiv:astro-ph/0602297

Ferrarese L, Côté P, Sánchez-Janssen R, Roediger J, McConnachie AW, Durrell PR, MacArthur LA, Blakeslee JP, Duc PA, Boissier S, Boselli A, Courteau S, Cuillandre JC, Emsellem E, Gwyn SDJ, Guhathakurta P, Jordán A, Lançon A, Liu C, Mei S, Mihos JC, Navarro JF, Peng EW, Puzia TH, Taylor JE, Toloba E, Zhang H (2016) The next generation virgo cluster survey (NGVS). XIII. The luminosity and mass function of galaxies in the core of the virgo cluster and the contribution from disrupted satellites. ApJ 824:10. https://doi.org/10.3847/0004-637X/824/1/10. arXiv:1604.06462

Filippenko AV, Ho LC (2003) A low-mass central black hole in the bulgeless Seyfert 1 Galaxy NGC 4395. ApJ 588(1):L13-L16. https://doi.org/10.1086/375361. arXiv:astro-ph/0303429

Foord A, Gallo E, Hodges-Kluck E, Miller BP, Baldassare VF, Gültekin K, Gnedin OY (2017) AGN activity in nucleated galaxies as measured by Chandra. ApJ 841(1):51. https://doi.org/10.3847/15384357/aa6d63. arXiv:1704.03882

Fragione G, Leigh NWC, Perna R (2019) Black hole and neutron star mergers in Galactic Nuclei: the role of triples. MNRAS. https://doi.org/10.1093/mnras/stz1803. arXiv:1903.09160

Freitag M, Gürkan MA, Rasio FA (2006a) Runaway collisions in young star clusters-II. Numerical results. MNRAS 368(1):141-161. https://doi.org/10.1111/j.1365-2966.2006.10096.x. arXiv:astro$\mathrm{ph} / 0503130$ 
Freitag M, Rasio FA, Baumgardt H (2006b) Runaway collisions in young star clusters-I. Methods and tests. MNRAS 368(1):121-140. https://doi.org/10.1111/j.1365-2966.2006.10095.x. arXiv:astro-ph/ 0503129

Fritz TK, Gillessen S, Dodds-Eden K, Lutz D, Genzel R, Raab W, Ott T, Pfuhl O, Eisenhauer F, YusefZadeh F (2011) Line derived infrared extinction toward the galactic center. ApJ 737(2):73. https:// doi.org/10.1088/0004-637X/737/2/73. arXiv:1105.2822

Fritz TK, Chatzopoulos S, Gerhard O, Gillessen S, Genzel R, Pfuhl O, Tacchella S, Eisenhauer F, Ott T (2016) The nuclear cluster of the milky way: total mass and luminosity. ApJ 821(1):44. https://doi. org/10.3847/0004-637X/821/1/44. arXiv:1406.7568

Fujii M, Iwasawa M, Funato Y, Makino J (2009) Trojan stars in the galactic center. ApJ 695(2):1421-1429. https://doi.org/10.1088/0004-637X/695/2/1421. arXiv:0807.2818

Gallagher JS, Goad JW, Mould J (1982) Structure of the M33 nucleus. ApJ 263:101-107. https://doi.org/ $10.1086 / 160484$

Gallazzi A, Charlot S, Brinchmann J, White SDM, Tremonti CA (2005) The ages and metallicities of galaxies in the local universe. MNRAS 362(1):41-58. https://doi.org/10.1111/j.1365-2966.2005. 09321.x. arXiv:astro-ph/0506539

Gallego-Cano E, Schödel R, Dong H, Nogueras-Lara F, Gallego-Calvente AT, Amaro-Seoane P, Baumgardt H (2018) The distribution of stars around the Milky Way's central black hole. I. Deep star counts. A\&A 609:A26. https://doi.org/10.1051/0004-6361/201730451. arXiv:1701.03816

Gallo E, Treu T, Marshall PJ, Woo JH, Leipski C, Antonucci R (2010) AMUSE-Virgo. II. Down-sizing in black hole accretion. ApJ 714(1):25-36. https://doi.org/10.1088/0004-637X/714/1/25. arXiv:1002. 3619

Gebhardt K, Lauer TR, Kormendy J, Pinkney J, Bower GA, Green R, Gull T, Hutchings JB, Kaiser ME, Nelson CH (2001) M33: a galaxy with no supermassive black hole. AJ 122(5):2469-2476. https:// doi.org/10.1086/323481. arXiv:astro-ph/0107135

Gebhardt K, Rich RM, Ho LC (2002) A 20,000 $\mathrm{M}_{\text {solar }}$ black hole in the stellar cluster G1. ApJ 578(1):L41-L45. https://doi.org/10.1086/342980. arXiv:astro-ph/0209313

Gebhardt K, Richstone D, Tremaine S, Lauer TR, Bender R, Bower G, Dressler A, Faber SM, Filippenko AV, Green R, Grillmair C, Ho LC, Kormendy J, Magorrian J, Pinkney J (2003) Axisymmetric dynamical models of the central regions of galaxies. ApJ 583(1):92-115. https://doi.org/10.1086/ 345081. arXiv:astro-ph/0209483

Gebhardt K, Rich RM, Ho LC (2005) An intermediate-mass black hole in the globular cluster G1: improved significance from new Keck and Hubble Space Telescope observations. ApJ 634(2):1093-1102. https://doi.org/10.1086/497023. arXiv:astro-ph/0508251

Gebhardt K, Adams J, Richstone D, Lauer TR, Faber SM, Gültekin K, Murphy J, Tremaine S (2011) The black hole mass in M87 from Gemini/NIFS adaptive optics observations. ApJ 729(2):119. https:// doi.org/10.1088/0004-637X/729/2/119. arXiv:1101.1954

Geha M, Guhathakurta P, van der Marel RP (2002) Internal dynamics, structure, and formation of Dwarf elliptical galaxies. I. A Keck/Hubble Space Telescope study of six Virgo cluster dwarf galaxies. AJ 124(6):3073-3087. https://doi.org/10.1086/344764

Genzel R, Thatte N, Krabbe A, Kroker H, Tacconi-Garman LE (1996) The dark mass concentration in the central parsec of the Milky Way. ApJ 472:153. https://doi.org/10.1086/178051

Genzel R, Eisenhauer F, Gillessen S (2010) The galactic center massive black hole and nuclear star cluster. Rev Mod Phys 82:3121-3195. https://doi.org/10.1103/RevModPhys.82.3121. arXiv:1006. 0064

Georgiev IY, Böker T (2014) Nuclear star clusters in 228 spiral galaxies in the HST/WFPC2 archive: catalogue and comparison to other stellar systems. MNRAS 441:3570-3590. https://doi.org/10. 1093/mnras/stu797. arXiv:1404.5956

Georgiev IY, Hilker M, Puzia TH, Goudfrooij P, Baumgardt H (2009) Globular cluster systems in nearby dwarf galaxies. II. Nuclear star clusters and their relation to massive Galactic globular clusters. MNRAS 396(2):1075-1085. https://doi.org/10.1111/j.1365-2966.2009.14776.x. arXiv:0903.2857

Georgiev IY, Böker T, Leigh N, Lützgendorf N, Neumayer N (2016) Masses and scaling relations for nuclear star clusters, and their co-existence with central black holes. MNRAS 457:2122-2138. https://doi.org/10.1093/mnras/stw093. arXiv:1601.02613

Gerhard O (2001) The galactic center HE I stars: remains of a dissolved young cluster? ApJ 546(1):L39_ L42. https://doi.org/10.1086/318054. arXiv:astro-ph/0005096

Ghez AM, Duchêne G, Matthews K, Hornstein SD, Tanner A, Larkin J, Morris M, Becklin EE, Salim S, Kremenek T (2003) The first measurement of spectral lines in a short-period star bound to the 
Galaxy's central black hole: a paradox of youth. ApJ 586(2):L127-L131. https://doi.org/10.1086/ 374804. arXiv:astro-ph/0302299

Gnedin OY, Ostriker JP, Tremaine S (2014) Co-evolution of galactic nuclei and globular cluster systems. ApJ 785(1):71. https://doi.org/10.1088/0004-637X/785/1/71. arXiv:1308.0021

Goerdt T, Moore B, Read JI, Stadel J, Zemp M (2006) Does the Fornax dwarf spheroidal have a central cusp or core? MNRAS 368(3):1073-1077. https://doi.org/10.1111/j.1365-2966.2006.10182.x. arXiv:astro-ph/0601404

Graham AW, Driver SP (2005) A concise reference to (Projected) Sérsic $\mathrm{R}^{1 / n}$ quantities, including concentration, profile slopes, petrosian indices, and kron magnitudes. PASA 22(2):118-127. https:// doi.org/10.1071/AS05001. arXiv:astro-ph/0503176

Graham AW, Guzmán R (2003) HST photometry of Dwarf elliptical galaxies in coma, and an explanation for the alleged structural dichotomy between dwarf and bright elliptical galaxies. AJ 125(6):2936-2950. https://doi.org/10.1086/374992. arXiv:astro-ph/0303391

Graham AW, Spitler LR (2009) Quantifying the coexistence of massive black holes and dense nuclear star clusters. MNRAS 397(4):2148-2162. https://doi.org/10.1111/j.1365-2966.2009.15118.x. arXiv: 0907.5250

Greene JE, Strader J, Ho LC (2019) Intermediate mass black holes. ARA\&A 58:1. arXiv:1911.09678

Guillard N, Emsellem E, Renaud F (2016) New insights on the formation of nuclear star clusters. MNRAS 461:3620-3629. https://doi.org/10.1093/mnras/stw1570. arXiv:1606.09537

Gullieuszik M, Greggio L, Falomo R, Schreiber L, Uslenghi M (2014) Probing the nuclear star cluster of galaxies with extremely large telescopes. A\&A 568:A89. https://doi.org/10.1051/0004-6361/ 201424279. arXiv: 1406.7818

Gültekin K, Richstone DO, Gebhardt K, Lauer TR, Pinkney J, Aller MC, Bender R, Dressler A, Faber SM, Filippenko AV, Green R, Ho LC, Kormendy J, Siopis C (2009) A quintet of black hole mass determinations. ApJ 695(2):1577-1590. https://doi.org/10.1088/0004-637X/695/2/1577. arXiv: 0901.4162

Gürkan MA, Freitag M, Rasio FA (2004) Formation of massive black holes in dense star clusters. I. Mass segregation and core collapse. ApJ 604(2):632-652. https://doi.org/10.1086/381968. arXiv:astro-ph/ 0308449

Habas R, Marleau FR, Duc PA, Durrell PR, Paudel S, Poulain M, Sánchez-Janssen R, Sreejith S, Ramasawmy J, Stemock B, Leach C, Cuillandre JC, Gwyn S, Agnello A, Bílek M, Fensch J, Müller O, Peng EW, van der Burg RFJ (2019) Newly discovered dwarf galaxies in the MATLAS low density fields. MNRAS. https://doi.org/10.1093/mnras/stz3045

Harris WE (1996) A catalog of parameters for globular clusters in the milky way. AJ 112:1487. https:// doi.org/10.1086/118116

Hartmann M, Debattista VP, Seth A, Cappellari M, Quinn TR (2011) Constraining the role of star cluster mergers in nuclear cluster formation: simulations confront integral-field data. MNRAS 418:2697-2714. https://doi.org/10.1111/j.1365-2966.2011.19659.x. arXiv:1103.5464

Hebbar PR, Heinke CO, Sivakoff GR, Shaw AW (2019) X-ray spectroscopy of the candidate AGNs in Henize 2-10 and NGC 4178: likely supernova remnants. MNRAS 485(4):5604-5615. https://doi. org/10.1093/mnras/stz553. arXiv:1902.08293

Hilker M, Infante L, Vieira G, Kissler-Patig M, Richtler T (1999) The central region of the Fornax cluster. II. Spectroscopy and radial velocities of member and background galaxies. A\&AS 134:75-86. https://doi.org/10.1051/aas:1999434. arXiv:astro-ph/9807144

Hilker M, Kayser A, Richtler T, Willemsen P (2004) The extended star formation history of $\omega$ Centauri. A\&A 422:L9-L12. https://doi.org/10.1051/0004-6361:20040188. arXiv:astro-ph/0406017

Holley-Bockelmann K, Gültekin K, Shoemaker D, Yunes N (2008) Gravitational wave recoil and the retention of intermediate-mass black holes. ApJ 686(2):829-837. https://doi.org/10.1086/591218. arXiv:0707.1334

Hopkins PF, Quataert E (2010a) How do massive black holes get their gas? MNRAS 407(3):1529-1564. https://doi.org/10.1111/j.1365-2966.2010.17064.x. arXiv:0912.3257

Hopkins PF, Quataert E (2010b) The nuclear stellar disc in Andromeda: a fossil from the era of black hole growth. MNRAS 405:L41-L45. https://doi.org/10.1111/j.1745-3933.2010.00855.x. arXiv:1002. 1079

Hopkins PF, Cox TJ, Dutta SN, Hernquist L, Kormendy J, Lauer TR (2009) Dissipation and extra light in galactic nuclei. II. “Cusp” ellipticals. ApJS 181(1):135-182. https://doi.org/10.1088/0067-0049/ 181/1/135. arXiv:0805.3533 
Hopkins PF, Murray N, Quataert E, Thompson TA (2010) A maximum stellar surface density in dense stellar systems. MNRAS 401(1):L19-L23. https://doi.org/10.1111/j.1745-3933.2009.00777.x. arXiv:0908.4088

Hopman C, Alexander T (2006) The effect of mass segregation on gravitational wave sources near massive black holes. ApJ 645(2):L133-L136. https://doi.org/10.1086/506273. arXiv:astro-ph/ 0603324

Hughes MA, Axon D, Atkinson J, Alonso-Herrero A, Scarlata C, Marconi A, Batcheldor D, Binney J, Capetti A, Carollo CM, Dressel L, Gerssen J, Macchetto D, Maciejewski W, Merrifield M, Ruiz M, Sparks W, Stiavelli M, Tsvetanov Z (2005) Nuclear properties of nearby spiral galaxies from Hubble Space Telescope NICMOS imaging and STIS spectroscopy. AJ 130:73-83. https://doi.org/ 10.1086/430531. arXiv:astro-ph/0503693

Hunt LK, Combes F, García-Burillo S, Schinnerer E, Krips M, Baker AJ, Boone F, Eckart A, Léon S, Neri R, Tacconi LJ (2008) Molecular gas in NUclei of GAlaxies (NUGA). IX. The decoupled bars and gas inflow in NGC 2782. A\&A 482(1):133-150. https://doi.org/10.1051/0004-6361:20078874. arXiv:0802.2775

Ibata R, Bellazzini M, Chapman SC, Dalessandro E, Ferraro F, Irwin M, Lanzoni B, Lewis GF, Mackey AD, Miocchi P, Varghese A (2009) Density and kinematic cusps in M54 at the heart of the sagittarius dwarf galaxy: evidence for A $10^{4} \mathrm{M}_{\text {sun }}$ black hole? ApJ 699(2):L169-L173. https://doi. org/10.1088/0004-637X/699/2/L169. arXiv:0906.4894

Ibata RA, Bellazzini M, Malhan K, Martin N, Bianchini P (2019) Identification of the long stellar stream of the prototypical massive globular cluster $\omega$ Centauri. Nat Astron 3:667-672. https://doi.org/10. 1038/s41550-019-0751-x. arXiv:1902.09544

Inayoshi K, Visbal E, Haiman Z (2019) The assembly of the first massive black holes. arXiv:1911.05791 (arXiv e-prints)

Janz J, Norris MA, Forbes DA, Huxor A, Romanowsky AJ, Frank MJ, Escudero CG, Faifer FR, Forte JC, Kannappan SJ, Maraston C, Brodie JP, Strader J, Thompson BR (2016) The AIMSS project-III. The stellar populations of compact stellar systems. MNRAS 456(1):617-632. https://doi.org/10.1093/ mnras/stv2636. arXiv:1511.03264

Jarrett TH, Chester T, Cutri R, Schneider S, Skrutskie M, Huchra JP (2000) 2MASS extended source catalog: overview and algorithms. AJ 119(5):2498-2531. https://doi.org/10.1086/301330. arXiv: astro-ph/0004318

Jarrett TH, Chester T, Cutri R, Schneider SE, Huchra JP (2003) The 2MASS large galaxy atlas. AJ 125(2):525-554. https://doi.org/10.1086/345794

Jennings ZG, Romanowsky AJ, Brodie JP, Janz J, Norris MA, Forbes DA, Martinez-Delgado D, Fagioli M, Penny SJ (2015) NGC 3628-UCD1: a possible $\omega$ Cen analog embedded in a stellar stream. ApJ 812(1):L10. https://doi.org/10.1088/2041-8205/812/1/L10. arXiv:1509.04710

Johnson CI, Pilachowski CA (2010) Chemical abundances for 855 giants in the globular cluster omega Centauri (NGC 5139). ApJ 722(2):1373-1410. https://doi.org/10.1088/0004-637X/722/2/1373. arXiv: 1008.2232

Jordán A, Peng EW, Blakeslee JP, Côté P, Eyheramendy S, Ferrarese L, Mei S, Tonry JL, West MJ (2009) The ACS virgo cluster survey XVI. Selection procedure and catalogs of globular cluster candidates. ApJS 180(1):54-66. https://doi.org/10.1088/0067-0049/180/1/54

Kacharov N, Neumayer N, Seth AC, Cappellari M, McDermid R, Walcher CJ, Böker T (2018) Stellar populations and star formation histories of the nuclear star clusters in six nearby galaxies. MNRAS 480(2):1973-1998. https://doi.org/10.1093/mnras/sty1985. arXiv:1807.08765

Kamann S, Husser TO, Dreizler S, Emsellem E, Weilbacher PM, Martens S, Bacon R, den Brok M, Giesers B, Krajnović D, Roth MM, Wendt M, Wisotzki L (2018) A stellar census in globular clusters with MUSE: the contribution of rotation to cluster dynamics studied with 200,000 stars. MNRAS 473(4):5591-5616. https://doi.org/10.1093/mnras/stx2719. arXiv:1710.07257

Karachentsev ID, Makarov DI, Kaisina EI (2013) Updated nearby galaxy catalog. AJ 145(4):101. https:// doi.org/10.1088/0004-6256/145/4/101. arXiv:1303.5328

Khan FM, Awais Mirza M, Holley-Bockelmann K (2020) Inward bound: the incredible journey of massive black holes as they pair and merge- - I. The effect of mass ratio in flattened rotating galactic nuclei. MNRAS 492:256-267. https://doi.org/10.1093/mnras/stz3360. arXiv:1911.07946

Kieffer TF, Bogdanović T (2016) Can star-disk collisions explain the missing red giants problem in the galactic center? ApJ 823(2):155. https://doi.org/10.3847/0004-637X/823/2/155. arXiv:1602.03527 
Kim WT, Elmegreen BG (2017) Nuclear spiral shocks and induced gas inflows in weak oval potentials. ApJ 841(1):L4. https://doi.org/10.3847/2041-8213/aa70a1. arXiv:1705.00863

Kim SC, Sung H, Park HS, Sung EC (2004) UBVI surface photometry of the spiral galaxy NGC 300 in the sculptor group. Chin J Astron Astrophys 4:299-310. https://doi.org/10.1088/1009-9271/4/4/299. arXiv:astro-ph/0404036

King I (1962) The structure of star clusters. I. An empirical density law. AJ 67:471. https://doi.org/10. $1086 / 108756$

King IR, Stanford SA, Crane P (1995) Far-UV properties of the nuclear region of M31. AJ 109:164. https://doi.org/10.1086/117264

Kirby EN, Cohen JG, Guhathakurta P, Cheng L, Bullock JS, Gallazzi A (2013) The universal stellar mass-stellar metallicity relation for dwarf galaxies. ApJ 779(2):102. https://doi.org/10.1088/0004637X/779/2/102. arXiv:1310.0814

Koleva M, de Rijcke S, Prugniel P, Zeilinger WW, Michielsen D (2009) Formation and evolution of dwarf elliptical galaxies-II. Spatially resolved star formation histories. MNRAS 396(4):2133-2151. https://doi.org/10.1111/j.1365-2966.2009.14820.x. arXiv:0903.4393

Koleva M, Prugniel P, De Rijcke S, Zeilinger WW (2011) Age and metallicity gradients in early-type galaxies: a dwarf-to-giant sequence. MNRAS 417(3):1643-1671. https://doi.org/10.1111/j.13652966.2011.19057.x. arXiv:1105.4809

Kormendy J (1985) Brightness profiles of the cores of bulges and elliptical galaxies. ApJ 292:L9-L13. https://doi.org/10.1086/184463

Kormendy J, Bender R (1999) The double nucleus and central black hole of M31. ApJ 522(2):772-792. https://doi.org/10.1086/307665

Kormendy J, Djorgovski S (1989) Surface photometry and the structure of elliptical galaxies. ARA\&A 27:235-277. https://doi.org/10.1146/annurev.aa.27.090189.001315

Kormendy J, Ho LC (2013) Coevolution (Or Not) of supermassive black holes and host galaxies. ARA\&A 51(1):511-653. https://doi.org/10.1146/annurev-astro-082708-101811. arXiv:1304.7762

Kormendy J, McClure RD (1993) The nucleus of M33. AJ 105:1793. https://doi.org/10.1086/116555

Kormendy J, Richstone D (1992) Evidence for a supermassive black hole in NGC 3115. ApJ 393:559. https://doi.org/10.1086/171528

Kormendy J, Fisher DB, Cornell ME, Bender R (2009) Structure and formation of elliptical and spheroidal galaxies. ApJS 182(1):216-309. https://doi.org/10.1088/0067-0049/182/1/216. arXiv: 0810.1681

Kormendy J, Drory N, Bender R, Cornell ME (2010) Bulgeless giant galaxies challenge our picture of galaxy formation by hierarchical clustering. ApJ 723(1):54-80. https://doi.org/10.1088/0004-637X/ 723/1/54. arXiv:1009.3015

Krabbe A, Genzel R, Drapatz S, Rotaciuc V (1991) A cluster of he i emission-line stars in the galactic center. ApJ 382:L19. https://doi.org/10.1086/186204

Krajnović D, Cappellari M, McDermid RM, Thater S, Nyland K, de Zeeuw PT, Falcón-Barroso J, Khochfar S, Kuntschner H, Sarzi M, Young LM (2018) A quartet of black holes and a missing duo: probing the low end of the $\mathrm{M}_{B H^{-}}$relation with the adaptive optics assisted integral-field spectroscopy. MNRAS 477(3):3030-3064. https://doi.org/10.1093/mnras/sty778. arXiv:1803.08055

Kruijssen JMD, Pfeffer JL, Reina-Campos M, Crain RA, Bastian N (2019) The formation and assembly history of the Milky Way revealed by its globular cluster population. MNRAS 486(3):3180-3202. https://doi.org/10.1093/mnras/sty1609. arXiv:1806.05680

Krumholz MR, McKee CF, Bland-Hawthorn J (2019) Star clusters across cosmic time. ARA\&A 57:227-303. https://doi.org/10.1146/annurev-astro-091918-104430. arXiv:1812.01615

Lacy JH, Townes CH, Geballe TR, Hollenbach DJ (1980) Observations of the motion and distribution of the ionized gas in the central parsec of the Galaxy. II. ApJ 241:132-146. https://doi.org/10.1086/ 158324

Lacy JH, Townes CH, Hollenbach DJ (1982) The nature of the central parsec of the Galaxy. ApJ 262:120-134. https://doi.org/10.1086/160402

Lahén N, Naab T, Johansson PH, Elmegreen B, Hu CY, Walch S (2019) The formation of low-metallicity globular clusters in dwarf galaxy mergers. ApJ 879(2):L18. https://doi.org/10.3847/2041-8213/ ab2a13. arXiv:1905.09840

Lamers HJGLM, Kruijssen JMD, Bastian N, Rejkuba M, Hilker M, Kissler-Patig M (2017) The difference in metallicity distribution functions of halo stars and globular clusters as a function of galaxy type. A tracer of globular cluster formation and evolution. A\&A 606:A85. https://doi.org/10. 1051/0004-6361/201731062. arXiv:1706.00939 
Larsen SS (1999) Young massive star clusters in nearby galaxies. II. Software tools, data reductions and cluster sizes. A\&AS 139:393-415. https://doi.org/10.1051/aas:1999509. arXiv:astro-ph/9907163

Lauer TR, Faber SM, Groth EJ, Shaya EJ, Campbell B, Code A, Currie DG, Baum WA, Ewald SP, Hester JJ, Holtzman JA, Kristian J, Light RM, Ligynds CR, O'Neil JEJ, Westphal JA (1993) Planetary camera observations of the double nucleus of M31. AJ 106:1436. https://doi.org/10.1086/116737

Lauer TR, Faber SM, Ajhar EA, Grillmair CJ, Scowen PA (1998) M32 \pm 1. AJ 116(5):2263-2286. https://doi.org/10.1086/300617. arXiv:astro-ph/9806277

Lauer TR, Faber SM, Gebhardt K, Richstone D, Tremaine S, Ajhar EA, Aller MC, Bender R, Dressler A, Filippenko AV, Green R, Grillmair CJ, Ho LC, Kormendy J, Magorrian J, Pinkney J, Siopis C (2005) The centers of early-type galaxies with Hubble Space Telescope. V. New WFPC2 photometry. AJ 129(5):2138-2185. https://doi.org/10.1086/429565. arXiv:astro-ph/0412040

Lauer TR, Bender R, Kormendy J, Rosenfield P, Green RF (2012) The cluster of blue stars surrounding the M31 nuclear black hole. ApJ 745(2):121. https://doi.org/10.1088/0004-637X/745/2/121. arXiv: 1112.1419

Launhardt R, Zylka R, Mezger PG (2002) The nuclear bulge of the Galaxy. III. Large-scale physical characteristics of stars and interstellar matter. A\&A 384:112-139. https://doi.org/10.1051/00046361:20020017. arXiv:astro-ph/0201294

Law-Smith J, MacLeod M, Guillochon J, Macias P, Ramirez-Ruiz E (2017) Low-mass white dwarfs with hydrogen envelopes as a missing link in the tidal disruption menu. ApJ 841(2):132. https://doi.org/ 10.3847/1538-4357/aa6ffb. arXiv:1701.08162

Leigh N, Böker T, Knigge C (2012) Nuclear star clusters and the stellar spheroids of their host galaxies. MNRAS 424(3):2130-2138. https://doi.org/10.1111/j.1365-2966.2012.21365.x. arXiv:1205.5033

Leigh NWC, Mastrobuono-Battisti A, Perets HB, Böker T (2014) Stellar dynamics in gas: the role of gas damping. MNRAS 441(2):919-932. https://doi.org/10.1093/mnras/stu622. arXiv:1404.0379

Leigh NWC, Antonini F, Stone NC, Shara MM, Merritt D (2016) On the origins of enigmatic stellar populations in Local Group galactic nuclei. MNRAS 463(2):1605-1623. https://doi.org/10.1093/ mnras/stw2018. arXiv:1608.02944

Leigh NWC, Geller AM, McKernan B, Ford KES, Mac Low MM, Bellovary J, Haiman Z, Lyra W, Samsing J, O'Dowd M, Kocsis B, Endlich S (2018) On the rate of black hole binary mergers in galactic nuclei due to dynamical hardening. MNRAS 474(4):5672-5683. https://doi.org/10.1093/ mnras/stx3134. arXiv:1711.10494

Levin Y, Beloborodov AM (2003) Stellar disk in the galactic center: a remnant of a dense accretion disk? ApJ 590(1):L33-L36. https://doi.org/10.1086/376675. arXiv:astro-ph/0303436

Lezhnin K, Vasiliev E (2016) Tidal disruption rates in non-spherical galactic nuclei formed by galaxy mergers. ApJ 831(1):84. https://doi.org/10.3847/0004-637X/831/1/84. arXiv:1609.00009

Li H, Gnedin OY (2019) Star cluster formation in cosmological simulations-III. Dynamical and chemical evolution. MNRAS 486(3):4030-4043. https://doi.org/10.1093/mnras/stz1114. arXiv: 1810.11036

Li H, Gnedin OY, Gnedin NY, Meng X, Semenov VA, Kravtsov AV (2017) Star cluster formation in cosmological simulations. I. Properties of young clusters. ApJ 834(1):69. https://doi.org/10.3847/ 1538-4357/834/1/69. arXiv:1608.03244

Light ES, Danielson RE, Schwarzschild M (1974) The nucleus of M31. ApJ 194:257-263. https://doi.org/ $10.1086 / 153241$

Lim S, Peng EW, Côté P, Sales LV, den Brok M, Blakeslee JP, Guhathakurta P (2018) The globular cluster systems of ultra-diffuse galaxies in the coma cluster. ApJ 862(1):82. https://doi.org/10.3847/ 1538-4357/aacb81. arXiv:1806.05425

Lindqvist M, Habing HJ, Winnberg A (1992) OH/IR stars close to the galactic centre. II. Their spatial and kinematics properties and the mass distribution within 5-100 pc from the galactic centre. A\&A 259:118-127

Lisker T, Grebel EK, Binggeli B, Glatt K (2007) Virgo cluster early-type dwarf galaxies with the sloan digital sky survey. III. Subpopulations: distributions, shapes, origins. ApJ 660(2):1186-1197. https:// doi.org/10.1086/513090. arXiv:astro-ph/0701429

Liu C, Peng EW, Côté P, Ferrarese L, Jordán A, Mihos JC, Zhang HX, Muñoz RP, Puzia TH, Lançon A, Gwyn S, Cuillandre JC, Blakeslee JP, Boselli A, Durrell PR, Duc PA, Guhathakurta P, MacArthur LA, Mei S, Sánchez-Janssen R, Xu H (2015) The next generation Virgo cluster survey. X. Properties of ultra-compact dwarfs in the M87, M49, and M60 regions. ApJ 812(1):34. https://doi.org/10.1088/ 0004-637X/812/1/34. arXiv:1508.07334 
Lockhart KE, Lu JR, Peiris HV, Rich RM, Bouchez A, Ghez AM (2018) A slowly precessing disk in the nucleus of M31 as the feeding mechanism for a central starburst. ApJ 854(2):121. https://doi.org/10. 3847/1538-4357/aaaa71. arXiv:1710.01394

Loose HH, Kruegel E, Tutukov A (1982) Bursts of star formation in the galactic centre. A\&A 105(2):342-350

Lotz JM, Telford R, Ferguson HC, Miller BW, Stiavelli M, Mack J (2001) Dynamical friction in de globular cluster systems. ApJ 552:572-581

Lu JR, Ghez AM, Hornstein SD, Morris MR, Becklin EE, Matthews K (2009) A disk of young stars at the galactic center as determined by individual stellar orbits. ApJ 690(2):1463-1487. https://doi.org/10. 1088/0004-637X/690/2/1463. arXiv:0808.3818

Lu JR, Do T, Ghez AM, Morris MR, Yelda S, Matthews K (2013) Stellar populations in the central 0.5 pc of the galaxy. II. The initial mass function. ApJ 764(2):155. https://doi.org/10.1088/0004-637X/764/ 2/155. arXiv:1301.0540

Lyubenova M, van den Bosch RCE, Côté P, Kuntschner H, van de Ven G, Ferrarese L, Jordán A, Infante L, Peng EW (2013) The complex nature of the nuclear star cluster in FCC 277. MNRAS 431(4):3364-3372. https://doi.org/10.1093/mnras/stt414. arXiv:1303.1210

Lyubenova M, Tsatsi A (2019) Nuclear angular momentum of early-type galaxies hosting nuclear star clusters. A\&A 629:A44. https://doi.org/10.1051/0004-6361/201833954. arXiv:1903.10918

Maciejewski W (2004) Nuclear spirals in galaxies: gas response to an asymmetric potential-II. Hydrodynamical models. MNRAS 354(3):892-904. https://doi.org/10.1111/j.1365-2966.2004. 08254.X. arXiv:astro-ph/0408100

Ma X, Grudić MY, Quataert E, Hopkins PF, Faucher-Giguère CA, Boylan-Kolchin M, Wetzel A, Kim Jh, Murray N, Kereš D (2019) Self-consistent proto-globular cluster formation in cosmological simulations of high-redshift galaxies. Mon. Not. R. Astron. Soc. 493(3):4315-4332. https://doi.org/ 10.1093/mnras/staa527. arXiv:1906.11261

Majewski SR, Nidever DL, Smith VV, Damke GJ, Kunkel WE, Patterson RJ, Bizyaev D, García Pérez AE (2012) Exploring halo substructure with giant stars: substructure in the local halo as seen in the grid giant star survey including extended tidal debris from $\omega$ Centauri. ApJ 747(2):L37. https://doi. org/10.1088/2041-8205/747/2/L37. arXiv:1202.1832

Maksym WP, Ulmer MP, Roth KC, Irwin JA, Dupke R, Ho LC, Keel WC, Adami C (2014) Deep spectroscopy of the $M_{V} \sim-14.8$ host galaxy of a tidal disruption flare in A1795. MNRAS 444(1):866-873. https://doi.org/10.1093/mnras/stu1485. arXiv:1407.6737

Mapelli M, Hayfield T, Mayer L, Wadsley J (2012) In situ formation of SgrA* stars via disk fragmentation: parent cloud properties and thermodynamics. ApJ 749(2):168. https://doi.org/10. 1088/0004-637X/749/2/168. arXiv:1202.0555

Marino AF, Milone AP, Piotto G, Cassisi S, D’Antona F, Anderson J, Aparicio A, Bedin LR, Renzini A, Villanova $\mathrm{S}$ (2012) The $\mathrm{C}+\mathrm{N}+\mathrm{O}$ abundance of $\omega$ Centauri giant stars: implications for the chemical-enrichment scenario and the relative ages of different stellar populations. ApJ 746(1):14. https://doi.org/10.1088/0004-637X/746/1/14. arXiv:1111.1891

Marino AF, Milone AP, Karakas AI, Casagrande L, Yong D, Shingles L, Da Costa G, Norris JE, Stetson PB, Lind K, Asplund M, Collet R, Jerjen H, Sbordone L, Aparicio A, Cassisi S (2015) Iron and s-elements abundance variations in NGC 5286: comparison with 'anomalous' globular clusters and Milky Way satellites. MNRAS 450(1):815-845. https://doi.org/10.1093/mnras/stv420. arXiv:1502. 07438

Martini P, Ho LC (2004) A population of massive globular clusters in NGC 5128. ApJ 610(1):233-246. https://doi.org/10.1086/421458. arXiv:astro-ph/0404003

Matthews LD (1899) Gallagher JS III (1997) B and V CCD photometry of southern. Extreme late-type spiral galaxies. AJ. https://doi.org/10.1086/118613. arXiv:astro-ph/9709145

Matthews LD, Gallagher JS III, Krist JE, Watson AM, Burrows CJ, Griffiths RE, Hester JJ, Trauger JT, Ballester GE, Clarke JT, Crisp D, Evans RW, Hoessel JG, Holtzman JA, Mould JR, Scowen PA, Stapelfeldt KR, Westphal JA (1999) WFPC2 observations of compact star cluster nuclei in lowluminosity spiral galaxies. AJ 118:208-235. https://doi.org/10.1086/300909. arXiv:astro-ph/ 9904205

Mayall NU, Aller LH (1942) The rotation of the spiral nebula Messier 33. ApJ 95:5. https://doi.org/10. $1086 / 144369$

McConnachie AW (2012) The observed properties of dwarf galaxies in and around the local group. AJ 144(1):4. https://doi.org/10.1088/0004-6256/144/1/4. arXiv:1204.1562 
McConnachie AW, Irwin MJ, Ferguson AMN, Ibata RA, Lewis GF, Tanvir N (2005) Distances and metallicities for 17 Local Group galaxies. MNRAS 356(3):979-997. https://doi.org/10.1111/j.13652966.2004.08514.x. arXiv:astro-ph/0410489

McGinn MT, Sellgren K, Becklin EE, Hall DNB (1989) Stellar kinematics in the galactic center. ApJ 338:824. https://doi.org/10.1086/167239

McKernan B, Ford KES, Bellovary J, Leigh NWC, Haiman Z, Kocsis B, Lyra W, Mac Low MM, Metzger B, O'Dowd M, Endlich S, Rosen DJ (2018) Constraining stellar-mass black hole mergers in AGN disks detectable with LIGO. ApJ 866(1):66. https://doi.org/10.3847/1538-4357/aadae5. arXiv: 1702.07818

McLaughlin DE (1999) The efficiency of globular cluster formation. AJ 117(5):2398-2427. https://doi. org/10.1086/300836. arXiv:astro-ph/9901283

McLaughlin DE, van der Marel RP (2005) Resolved massive star clusters in the milky way and its satellites: brightness profiles and a catalog of fundamental parameters. ApJS 161(2):304-360. https://doi.org/10.1086/497429. arXiv:astro-ph/0605132

Meadows N, Navarro JF, Santos-Santos I, Benítez-Llambay A, Frenk C (2019) Cusp or core? Revisiting the globular cluster timing problem in Fornax. MNRAS. https://doi.org/10.1093/mnras/stz3280

Merritt D (2009) Evolution of nuclear star clusters. ApJ 694:959-970. https://doi.org/10.1088/0004637X/694/2/959. arXiv:0802.3186

Merritt D (2010) The distribution of stars and stellar remnants at the galactic center. ApJ 718(2):739-761. https://doi.org/10.1088/0004-637X/718/2/739. arXiv:0909.1318

Merritt D, Alexander T, Mikkola S, Will CM (2011) Stellar dynamics of extreme-mass-ratio inspirals. Phys Rev D 84(4):044024. https://doi.org/10.1103/PhysRevD.84.044024. arXiv:1102.3180

Mezcua M (2017) Observational evidence for intermediate-mass black holes. Int J Mod Phys D 26(11):1730021. https://doi.org/10.1142/S021827181730021X. arXiv:1705.09667

Mezcua M, Civano F, Fabbiano G, Miyaji T, Marchesi S (2016) A population of intermediate-mass black holes in dwarf starburst galaxies up to redshift $=1.5$. ApJ 817(1):20. https://doi.org/10.3847/0004637X/817/1/20. arXiv:1511.05844

Mezcua M, Civano F, Marchesi S, Suh H, Fabbiano G, Volonteri M (2018) Intermediate-mass black holes in dwarf galaxies out to redshift $\sim 2.4$ in the Chandra COSMOS-legacy survey. MNRAS 478(2):2576-2591. https://doi.org/10.1093/mnras/sty1163. arXiv:1802.01567

Mieske S, Frank MJ, Baumgardt H, Lützgendorf N, Neumayer N, Hilker M (2013) On central black holes in ultra-compact dwarf galaxies. A\&A 558:A14. https://doi.org/10.1051/0004-6361/201322167. arXiv: 1308.1398

Mihos JC, Hernquist L (1994) Dense stellar cores in merger remnants. ApJ 437:L47. https://doi.org/10. $1086 / 187679$

Miller MC, Davies MB (2012) An upper limit to the velocity dispersion of relaxed stellar systems without massive black holes. ApJ 755(1):81. https://doi.org/10.1088/0004-637X/755/1/81. arXiv:1206.6167

Miller MC, Hamilton DP (2002) Production of intermediate-mass black holes in globular clusters. MNRAS 330(1):232-240. https://doi.org/10.1046/j.1365-8711.2002.05112.x. arXiv:astro-ph/ 0106188

Miller MC, Lauburg VM (2009) Mergers of stellar-mass black holes in nuclear star clusters. ApJ 692(1):917-923. https://doi.org/10.1088/0004-637X/692/1/917. arXiv:0804.2783

Miller BW, Lotz JM (2007) The globular cluster luminosity function and specific frequency in dwarf elliptical galaxies. ApJ 670(2):1074-1089. https://doi.org/10.1086/522323. arXiv:0708.2511

Miller BP, Gallo E, Greene JE, Kelly BC, Treu T, Woo JH, Baldassare V (2015) X-ray constraints on the local supermassive black hole occupation fraction. ApJ 799(1):98. https://doi.org/10.1088/0004637X/799/1/98. arXiv:1403.4246

Miller-Jones JCA, Wrobel JM, Sivakoff GR, Heinke CO, Miller RE, Plotkin RM, Di Stefano R, Greene JE, Ho LC, Joseph TD, Kong AKH, Maccarone TJ (2012) The absence of radio emission from the globular cluster G1. ApJ 755(1):L1. https://doi.org/10.1088/2041-8205/755/1/L1. arXiv:1206.5729

Milone AP, Piotto G, Renzini A, Marino AF, Bedin LR, Vesperini E, D’Antona F, Nardiello D, Anderson J, King IR, Yong D, Bellini A, Aparicio A, Barbuy B, Brown TM, Cassisi S, Ortolani S, Salaris M, Sarajedini A, van der Marel RP (2017) The Hubble Space Telescope UV legacy survey of galactic globular clusters-IX. The Atlas of multiple stellar populations. MNRAS 464(3):3636-3656. https://doi.org/10.1093/mnras/stw2531. arXiv:1610.00451

Milosavljević M (2004) On the origin of nuclear star clusters in late-type spiral galaxies. ApJ 605(1):L13-L16. https://doi.org/10.1086/420696. arXiv:astro-ph/0310574 
Milosavljević M, Merritt D (2001) Formation of galactic nuclei. ApJ 563(1):34-62. https://doi.org/10. 1086/323830. arXiv:astro-ph/0103350

Milosavljević M, Merritt D, Ho LC (2006) Contribution of stellar tidal disruptions to the X-ray luminosity function of active galaxies. ApJ 652(1):120-125. https://doi.org/10.1086/508134. arXiv:astro-ph/ 0602289

Misgeld I, Hilker M (2011) Families of dynamically hot stellar systems over 10 orders of magnitude in mass. MNRAS 414(4):3699-3710. https://doi.org/10.1111/j.1365-2966.2011.18669.x. arXiv:1103. 1628

Monaco L, Bellazzini M, Ferraro FR, Pancino E (2005) The central density cusp of the Sagittarius dwarf spheroidal galaxy. MNRAS 356:1396-1402. https://doi.org/10.1111/j.1365-2966.2004.08579.x. arXiv:astro-ph/0411107

Monaco L, Saviane I, Perina S, Bellazzini M, Buzzoni A, Federici L, Fusi Pecci F, Galleti S (2009) The young stellar population at the center of NGC 205. A\&A 502(2):L9-L12. https://doi.org/10.1051/ 0004-6361/200912412. arXiv:0907.0029

Moran EC, Shahinyan K, Sugarman HR, Vélez DO, Eracleous M (2014) Black holes at the centers of nearby dwarf galaxies. AJ 148(6):136. https://doi.org/10.1088/0004-6256/148/6/136. arXiv:1408. 4451

Morelli L, Cesetti M, Corsini EM, Pizzella A, Dalla Bontà E, Sarzi M, Bertola F (2010) Multiband photometric decomposition of nuclear stellar disks. A\&A 518:A32. https://doi.org/10.1051/00046361/201014285. arXiv:1004.2190

Mucciarelli A, Bellazzini M, Ibata R, Romano D, Chapman SC, Monaco L (2017) Chemical abundances in the nucleus of the Sagittarius dwarf spheroidal galaxy. A\&A 605:A46. https://doi.org/10.1051/ 0004-6361/201730707. arXiv:1705.03251

Naiman JP, Ramirez-Ruiz E, Debuhr J, Ma CP (2015) The role of nuclear star clusters in enhancing supermassive black hole feeding rates during galaxy mergers. ApJ 803(2):81. https://doi.org/10. 1088/0004-637X/803/2/81. arXiv:1410.7381

Nayakshin S, Wilkinson MI, King A (2009) Competitive feedback in galaxy formation. MNRAS 398(1):L54-L57. https://doi.org/10.1111/j.1745-3933.2009.00709.x. arXiv:0907.1002

Ness M, Freeman K, Athanassoula E, Wylie-de-Boer E, Bland-Hawthorn J, Asplund M, Lewis GF, Yong D, Lane RR, Kiss LL (2013) ARGOS-III. Stellar populations in the galactic bulge of the milky way. MNRAS 430(2):836-857. https://doi.org/10.1093/mnras/sts629. arXiv:1212.1540

Neumayer N, Walcher CJ (2012) Are nuclear star clusters the precursors of massive black holes? Adv Astron 2012:709038. https://doi.org/10.1155/2012/709038. arXiv:1201.4950

Neumayer N, Walcher CJ, Andersen D, Sánchez SF, Böker T, Rix HW (2011) Two-dimensional H $\alpha$ kinematics of bulgeless disc galaxies. MNRAS 413:1875-1888. https://doi.org/10.1111/j.13652966.2011.18266.x. arXiv:1101.5154

Nguyen DD, Seth AC, Reines AE, den Brok M, Sand D, McLeod B (2014) Extended structure and fate of the nucleus in Henize 2-10. ApJ 794(1):34. https://doi.org/10.1088/0004-637X/794/1/34. arXiv: 1408.4446

Nguyen DD, Seth AC, den Brok M, Neumayer N, Cappellari M, Barth AJ, Caldwell N, Williams BF, Binder B (2017) Improved dynamical constraints on the mass of the central black hole in NGC 404. ApJ 836(2):237. https://doi.org/10.3847/1538-4357/aa5cb4. arXiv:1610.09385

Nguyen DD, Seth AC, Neumayer N, Kamann S, Voggel KT, Cappellari M, Picotti A, Nguyen PM, Böker T, Debattista V, Caldwell N, McDermid R, Bastian N, Ahn CC, Pechetti R (2018) Nearby early-type galactic nuclei at high resolution: dynamical black hole and nuclear star cluster mass measurements. ApJ 858:118. https://doi.org/10.3847/1538-4357/aabe28. arXiv:1711.04314

Nguyen DD, Seth AC, Neumayer N, Iguchi S, Cappellari M, Strader J, Chomiuk L, Tremou E, Pacucci F, Nakanishi K (2019) Improved dynamical constraints on the masses of the central black holes in nearby low-mass early-type galactic nuclei and the first black hole determination for NGC 205. ApJ 872(1):104. https://doi.org/10.3847/1538-4357/aafe7a. arXiv:1901.05496

Nieto JL, Auriere M (1982) Optical structure of the nucleus of M33. A\&A 108(2):334-338

Nogueras-Lara F, Schödel R, Dong H, Najarro F, Gallego-Calvente AT, Hilker M, Gallego-Cano E, Nishiyama S, Neumayer N, Feldmeier-Krause A (2018) Star formation history and metallicity in the Galactic inner bulge revealed by the red giant branch bump. A\&A 620:A83. https://doi.org/10.1051/ 0004-6361/201833518. arXiv:1809.07627

Nogueras-Lara F, Schödel R, Najarro F, Gallego-Calvente AT, Gallego-Cano E, Shahzamanian B, Neumayer N (2019b) Variability of the near-infrared extinction curve towards the Galactic centre. A\&A 630:L3. https://doi.org/10.1051/0004-6361/201936322 
Nogueras-Lara F, Schödel R, Gallego-Calvente AT, Gallego-Cano E, Shahzamanian B, Dong H, Neumayer N, Hilker M, Najarro F, Nishiyama S, Feldmeier-Krause A, Girard JHV, Cassisi S (2019a) The nuclear disc of the Milky Way: early formation, long quiescence, and starburst activity one billion years ago. Nat Astron. arXiv:1910.06968

Norris JE, Freeman KC, Mighell KJ (1996) The Giant Branch of omega Centauri. V. The calcium abundance distribution. ApJ 462:241. https://doi.org/10.1086/177145

Norris MA, Kannappan SJ, Forbes DA, Romanowsky AJ, Brodie JP, Faifer FR, Huxor A, Maraston C, Moffett AJ, Penny SJ (2014) The AIMSS project-I. Bridging the star cluster-galaxy divide. MNRAS 443(2):1151-1172. https://doi.org/10.1093/mnras/stu1186. arXiv:1406.6065

Norris MA, Escudero CG, Faifer FR, Kannappan SJ, Forte JC, van den Bosch RCE (2015) An extended star formation history in an ultra-compact dwarf. MNRAS 451(4):3615-3626. https://doi.org/10. 1093/mnras/stv1221

Noyola E, Gebhardt K, Kissler-Patig M, Lützgendorf N, Jalali B, de Zeeuw PT, Baumgardt H (2010) Very large telescope kinematics for omega centauri: further support for a central black hole. ApJ 719(1):L60-L64. https://doi.org/10.1088/2041-8205/719/1/L60. arXiv:1007.4559

Oh KS, Lin DNC (2000) Nucleation of dwarf galaxies in the virgo cluster. ApJ 543(2):620-633. https:// doi.org/10.1086/317118

Ordenes-Briceño Y, Puzia TH, Eigenthaler P, Taylor MA, Muñoz RP, Zhang H, Alamo-Martínez K, Ribbeck KX, Grebel EK, Ángel S, Côté P, Ferrarese L, Hilker M, Lançon A, Mieske S, Miller BW, Rong Y, Sánchez-Janssen R (2018) The Next Generation Fornax Survey (NGFS). IV. Mass and age bimodality of nuclear clusters in the Fornax core region. ApJ 860:4. https://doi.org/10.3847/15384357/aac1b8. arXiv:1805.00491

Panamarev T, Just A, Spurzem R, Berczik P, Wang L, Arca Sedda M (2019) Direct N-body simulation of the Galactic centre. MNRAS 484(3):3279-3290. https://doi.org/10.1093/mnras/stz208. arXiv:1805. 02153

Paudel S, Lisker T, Kuntschner H (2011) Nuclei of early-type dwarf galaxies: insights from stellar populations. MNRAS 413(3):1764-1776. https://doi.org/10.1111/j.1365-2966.2011.18256.x. arXiv: 1012.4092

Paumard T, Genzel R, Martins F, Nayakshin S, Beloborodov AM, Levin Y, Trippe S, Eisenhauer F, Ott T, Gillessen S (2006) The two young star disks in the central parsec of the galaxy: properties, dynamics, and formation. ApJ 643(2):1011-1035. https://doi.org/10.1086/503273. arXiv:astro-ph/ 0601268

Pechetti R, Seth A, Cappellari M, McDermid R, den Brok M, Mieske S, Strader J (2017) Detection of enhanced central mass-to-light ratios in low-mass early-type galaxies: evidence for black holes? ApJ 850(1):15. https://doi.org/10.3847/1538-4357/aa9021. arXiv:1709.09172

Pechetti R, Seth A, Neumayer N, Georgiev I, Kacharov N, den Brok M (2019) Luminosity models and density profiles for nuclear star clusters for a nearby volume-limited sample of 29 galaxies. arXiv: 1911.09686 (arXiv e-prints)

Peiris HV, Tremaine S (2003) Eccentric-disk models for the nucleus of M31. ApJ 599(1):237-257. https://doi.org/10.1086/378638. arXiv:astro-ph/0307412

Peng CY (2002) Assessing formation scenarios for the double nucleus of M31 using two-dimensional image decomposition. AJ 124(1):294-309. https://doi.org/10.1086/340958. arXiv:astro-ph/0204184

Peterson BM, Bentz MC, Desroches LB, Filippenko AV, Ho LC, Kaspi S, Laor A, Maoz D, Moran EC, Pogge RW, Quillen AC (2005) Multiwavelength monitoring of the dwarf Seyfert 1 Galaxy NGC 4395. I. A reverberation-based measurement of the black hole mass. ApJ 632(2):799-808. https:// doi.org/10.1086/444494. arXiv:astro-ph/0506665

Pfeffer J, Baumgardt H (2013) Ultra-compact dwarf galaxy formation by tidal stripping of nucleated dwarf galaxies. MNRAS 433:1997-2005. https://doi.org/10.1093/mnras/stt867. arXiv:1305.3656

Pfeffer J, Griffen BF, Baumgardt H, Hilker M (2014) Contribution of stripped nuclear clusters to globular cluster and ultracompact dwarf galaxy populations. MNRAS 444:3670-3683. https://doi.org/10. 1093/mnras/stu1705. arXiv:1408.4467

Pfeffer J, Hilker M, Baumgardt H, Griffen BF (2016) Constraining ultracompact dwarf galaxy formation with galaxy clusters in the local universe. MNRAS 458(3):2492-2508. https://doi.org/10.1093/ mnras/stw498. arXiv:1603.00032

Pfeffer J, Kruijssen JMD, Crain RA, Bastian N (2018) The E-MOSAICS project: simulating the formation and co-evolution of galaxies and their star cluster populations. MNRAS 475(4):4309-4346. https://doi.org/10.1093/mnras/stx3124. arXiv:1712.00019 
Pfuhl O, Fritz TK, Zilka M, Maness H, Eisenhauer F, Genzel R, Gillessen S, Ott T, Dodds-Eden K, Sternberg A (2011) The star formation history of the Milky Way's nuclear star cluster. ApJ 741(2):108. https://doi.org/10.1088/0004-637X/741/2/108. arXiv:1110.1633

Phillips AC, Illingworth GD, MacKenty JW, Franx M (1996) Nuclei of nearby disk galaxies. I. A Hubble Space Telescope imaging survey. AJ 111:1566. https://doi.org/10.1086/117896

Portegies Zwart SF, McMillan SLW (2002) The runaway growth of intermediate-mass black holes in dense star clusters. ApJ 576(2):899-907. https://doi.org/10.1086/341798. arXiv:astro-ph/0201055

Portegies Zwart SF, Baumgardt H, Hut P, Makino J, McMillan SLW (2004) Formation of massive black holes through runaway collisions in dense young star clusters. Nature 428(6984):724-726. https:// doi.org/10.1038/nature02448. arXiv:astro-ph/0402622

Querejeta M, Meidt SE, Schinnerer E, Cisternas M, Muñoz-Mateos JC, Sheth K, Knapen J, van de Ven G, Norris MA, Peletier R, Laurikainen E, Salo H, Holwerda BW, Athanassoula E, Bosma A, Groves B, Ho LC, Gadotti DA, Zaritsky D, Regan M, Hinz J, Gil de Paz A, Menendez-Delmestre K, Seibert M, Mizusawa T, Kim T, Erroz-Ferrer S, Laine J, Comerón S (2015) The spitzer survey of stellar structure in galaxies $\left(\mathrm{S}^{4} \mathrm{G}\right)$ : precise stellar mass distributions from automated dust correction at 3.6 $\mu \mathrm{m}$. ApJS 219(1):5. https://doi.org/10.1088/0067-0049/219/1/5. arXiv:1410.0009

Quinlan GD, Hernquist L (1997) The dynamical evolution of massive black hole binaries-II. Selfconsistent N-body integrations. New A 2(6):533-554. https://doi.org/10.1016/S13841076(97)00039-0. arXiv:astro-ph/9706298

Quinlan GD, Shapiro SL (1987) The collapse of dense star clusters to supermassive black holes: binaries and gravitational radiation. ApJ 321:199. https://doi.org/10.1086/165624

Ravindranath S, Ho LC, Peng CY, Filippenko AV, Sargent WLW (2001) Central structural parameters of early-type galaxies as viewed with NICMOS on the Hubble Space Telescope. AJ 122(2):653-678. https://doi.org/10.1086/321175. arXiv:astro-ph/0105390

Reaves G (1983) A catalog of dwarf galaxies in Virgo. ApJS 53:375-395. https://doi.org/10.1086/190895

Redman RO, Shirley EG (1937) Photometry of the Andromeda nebula, M 31'. MNRAS 97:416. https:// doi.org/10.1093/mnras/97.6.416

Rees MJ (1988) Tidal disruption of stars by black holes of $10^{6}-10^{8}$ solar masses in nearby galaxies. Nature 333(6173):523-528. https://doi.org/10.1038/333523a0

Reines AE, Volonteri M (2015) Relations between central black hole mass and total galaxy stellar mass in the local universe. ApJ 813(2):82. https://doi.org/10.1088/0004-637X/813/2/82. arXiv:1508.06274

Reines AE, Sivakoff GR, Johnson KE, Brogan CL (2011) An actively accreting massive black hole in the dwarf starburst galaxy Henize2-10. Nature 470(7332):66-68. https://doi.org/10.1038/nature09724. arXiv:1101.1309

Reines AE, Greene JE, Geha M (2013) Dwarf galaxies with optical signatures of active massive black holes. ApJ 775(2):116. https://doi.org/10.1088/0004-637X/775/2/116. arXiv:1308.0328

Reines AE, Reynolds MT, Miller JM, Sivakoff GR, Greene JE, Hickox RC, Johnson KE (2016) Deep Chandra observations of the compact starburst galaxy Henize 2-10: X-rays from the massive black hole. ApJ 830(2):L35. https://doi.org/10.3847/2041-8205/830/2/L35. arXiv:1610.01598

Reines A, Condon J, Darling J, Greene J (2019) A new sample of (wandering) massive black holes in dwarf galaxies from high resolution radio observations. ApJ 888(1):36. https://doi.org/10.3847/ 1538-4357/ab4999. arXiv: 1909.04670

Renaud F (2018) Star clusters in evolving galaxies. New A Rev 81:1-38. https://doi.org/10.1016/j.newar. 2018.03.001. arXiv:1801.04278

Rich RM, Ryde N, Thorsbro B, Fritz TK, Schultheis M, Origlia L, Jönsson H (2017) Detailed abundances for the old population near the galactic center. I. Metallicity distribution of the nuclear star cluster. AJ 154(6):239. https://doi.org/10.3847/1538-3881/aa970a. arXiv:1710.08477

Richstone D, Bower G, Dressler A (1990) Constraints on the mass distribution near the centers of M31 and M32. ApJ 353:118. https://doi.org/10.1086/168596

Rieke GH, Rieke MJ (1988) Stellar velocities and the mass distribution in the galactic center. ApJ 330:L33. https://doi.org/10.1086/185199

Roediger JC, Courteau S (2015) On the uncertainties of stellar mass estimates via colour measurements. MNRAS 452(3):3209-3225. https://doi.org/10.1093/mnras/stv1499. arXiv:1507.03016

Romanishin W, Strom KM, Strom SE (1983) A study of low surface brightness spiral galaxies. II Optical surface photometry, infrared photometry, and H II region spectrophotometry. ApJS 53:105-128. https://doi.org/10.1086/190886 
Rossa J, van der Marel RP, Böker T, Gerssen J, Ho LC, Rix HW, Shields JC, Walcher CJ (2006) Hubble Space Telescope STIS spectra of nuclear star clusters in spiral galaxies: dependence of age and mass on Hubble type. AJ 132:1074-1099. https://doi.org/10.1086/505968. arXiv:astro-ph/0604140

Rusli SP, Thomas J, Erwin P, Saglia RP, Nowak N, Bender R (2011) The central black hole mass of the high- $\sigma$ but low-bulge-luminosity lenticular galaxy NGC 1332. MNRAS 410(2):1223-1236. https:// doi.org/10.1111/j.1365-2966.2010.17610.x. arXiv:1009.0515

Ryde N, Fritz TK, Rich RM, Thorsbro B, Schultheis M, Origlia L, Chatzopoulos S (2016) Detailed abundance analysis of a metal-poor giant in the galactic center. ApJ 831(1):40. https://doi.org/10. 3847/0004-637X/831/1/40. arXiv:1608.07562

Saglia RP, Fabricius M, Bender R, Montalto M, Lee CH, Riffeser A, Seitz S, Morganti L, Gerhard O, Hopp U (2010) The old and heavy bulge of M 31. I. Kinematics and stellar populations. A\&A 509:A61. https://doi.org/10.1051/0004-6361/200912805. arXiv:0910.5590

Saglia RP, Opitsch M, Erwin P, Thomas J, Beifiori A, Fabricius M, Mazzalay X, Nowak N, Rusli SP, Bender R (2016) The SINFONI black hole survey: the black hole fundamental plane revisited and the paths of (co)evolution of supermassive black holes and bulges. ApJ 818(1):47. https://doi.org/10. 3847/0004-637X/818/1/47. arXiv:1601.00974

Sánchez-Janssen R, Côté P, Ferrarese L, Peng EW, Roediger J, Blakeslee JP, Emsellem E, Puzia TH, Spengler C, Taylor J (2019a) The next generation virgo cluster survey. XXIII. Fundamentals of nuclear star clusters over seven decades in galaxy mass. ApJ 878(1):18. https://doi.org/10.3847/ 1538-4357/aaf4fd. arXiv:1812.01019

Sánchez-Janssen R, Puzia TH, Ferrarese L, Côté P, Eigenthaler P, Miller B, Ordenes-Briceño Y, Peng EW, Ribbeck KX, Roediger J (2019b) How nucleation and luminosity shape faint dwarf galaxies. MNRAS 486(1):L1-L5. https://doi.org/10.1093/mnrasl/slz008. arXiv:1901.04509

Sandage A (1961) The Hubble atlas of galaxies. Carnegie Institution, Washington

Sandage A, Bedke J (1994) The carnegie atlas of galaxies, vol I, II. Carnegie Institution, Washington

Sarajedini VL, Green RF, Griffiths RE, Ratnatunga K (1996) Compact nuclei in moderately redshifted galaxies. ApJ 471:L15. https://doi.org/10.1086/310333. arXiv:astro-ph/9608180

Sarzi M, Rix HW, Shields JC, Rudnick G, Ho LC, McIntosh DH, Filippenko AV, Sargent WLW (2001) Supermassive black holes in bulges. ApJ 550(1):65-74. https://doi.org/10.1086/319724. arXiv:astro$\mathrm{ph} / 0010240$

Satyapal S, Vega D, Dudik RP, Abel NP, Heckman T (2008) Spitzer uncovers active galactic nuclei missed by optical surveys in seven late-type galaxies. ApJ 677(2):926-942. https://doi.org/10.1086/ 529014. arXiv:0801.2759

Satyapal S, Böker T, Mcalpine W, Gliozzi M, Abel NP, Heckman T (2009) The incidence of active galactic nuclei in pure disk galaxies: the Spitzer view. ApJ 704(1):439-452. https://doi.org/10.1088/ 0004-637X/704/1/439. arXiv:0908.1820

Scarlata C, Stiavelli M, Hughes MA, Axon D, Alonso-Herrero A, Atkinson J, Batcheldor D, Binney J, Capetti A, Carollo CM, Dressel L, Gerssen J, Macchetto D, Maciejewski W, Marconi A, Merrifield M, Ruiz M, Sparks W, Tsvetanov Z, van der Marel RP (2004) Nuclear properties of a sample of nearby spiral galaxies from Hubble Space Telescope STIS imaging. AJ 128:1124-1137. https://doi. org/10.1086/423036. arXiv:astro-ph/0408435

Schiavon RP (2007) Population synthesis in the blue. IV. Accurate model predictions for lick indices and UBV colors in single stellar populations. ApJS 171(1):146-205. https://doi.org/10.1086/511753. arXiv:astro-ph/0611464

Schinnerer E, Böker T, Emsellem E, Lisenfeld U (2006) Molecular gas dynamics in NGC 6946: a bardriven nuclear starburst "Caught in the Act". ApJ 649(1):181-200. https://doi.org/10.1086/506265. arXiv:astro-ph/0605702

Schinnerer E, Böker T, Emsellem E, Downes D (2007) Bar-driven mass build-up within the central 50 pc of NGC 6946. A\&A 462(3):L27-L30. https://doi.org/10.1051/0004-6361:20066711. arXiv:astro-ph/ 0701599

Schödel R, Feldmeier A, Kunneriath D, Stolovy S, Neumayer N, Amaro-Seoane P, Nishiyama S (2014a) Surface brightness profile of the Milky Way's nuclear star cluster. A\&A 566:A47. https://doi.org/10. 1051/0004-6361/201423481. arXiv:1403.6657

Schödel R, Feldmeier A, Neumayer N, Meyer L, Yelda S (2014b) The nuclear cluster of the Milky Way: our primary testbed for the interaction of a dense star cluster with a massive black hole. Class Quant Gravit 31(24):244007. https://doi.org/10.1088/0264-9381/31/24/244007. arXiv:1411.4504

Schödel R, Gallego-Cano E, Dong H, Nogueras-Lara F, Gallego-Calvente AT, Amaro-Seoane P, Baumgardt H (2018) The distribution of stars around the Milky Way's central black hole. II. Diffuse 
light from sub-giants and dwarfs. A\&A 609:A27. https://doi.org/10.1051/0004-6361/201730452. arXiv:1701.03817

Schultheis M, Rich RM, Origlia L, Ryde N, Nandakumar G, Thorsbro B, Neumayer N (2019) The inner two degrees of the Milky Way. Evidence of a chemical difference between the Galactic Center and the surrounding inner bulge stellar populations. Astron Astrophys 627:A152. https://doi.org/10. 1051/0004-6361/201935772. arXiv:1906.07985

Scott N, Graham AW (2013) Updated mass scaling relations for nuclear star clusters and a comparison to supermassive black holes. ApJ 763:76. https://doi.org/10.1088/0004-637X/763/2/76. arXiv:1205. 5338

Secunda A, Bellovary J, Mac Low MM, Ford KES, McKernan B, Leigh NWC, Lyra W, Sándor Z (2019) Orbital migration of interacting stellar mass black holes in disks around supermassive black holes. ApJ 878(2):85. https://doi.org/10.3847/1538-4357/ab20ca. arXiv:1807.02859

Seigar M, Carollo CM, Stiavelli M, de Zeeuw PT, Dejonghe H (2002) Spiral galaxies with HST/ NICMOS. II. Isophotal fits and nuclear cusp slopes. AJ 123:184-194. https://doi.org/10.1086/ 324730. arXiv:astro-ph/0110282

Sérsic JL (1968) Atlas de galaxias australes. Observatorio Astronomico, Cordoba

Seth AC, Dalcanton JJ, Hodge PW, Debattista VP (2006) Clues to nuclear star cluster formation from edge-on spirals. AJ 132:2539-2555. https://doi.org/10.1086/508994. arXiv:astro-ph/0609302

Seth A, Agüeros M, Lee D, Basu-Zych A (2008a) The coincidence of nuclear star clusters and active galactic nuclei. ApJ 678:116-130. https://doi.org/10.1086/528955. arXiv:0801.0439

Seth AC, Blum RD, Bastian N, Caldwell N, Debattista VP (2008b) The rotating nuclear star cluster in NGC 4244. ApJ 687(2):997-1003. https://doi.org/10.1086/591935. arXiv:0807.3044

Seth AC, Cappellari M, Neumayer N, Caldwell N, Bastian N, Olsen K, Blum RD, Debattista VP, McDermid R, Puzia T, Stephens A (2010) The NGC 404 nucleus: star cluster and possible intermediate-mass black hole. ApJ 714(1):713-731. https://doi.org/10.1088/0004-637X/714/1/713. arXiv:1003.0680

Seth AC, van den Bosch R, Mieske S, Baumgardt H, Brok MD, Strader J, Neumayer N, Chilingarian I, Hilker M, McDermid R, Spitler L, Brodie J, Frank MJ, Walsh JL (2014) A supermassive black hole in an ultra-compact dwarf galaxy. Nature 513:398-400. https://doi.org/10.1038/nature13762. arXiv: 1409.4769

Shapiro KL, Cappellari M, de Zeeuw T, McDermid RM, Gebhardt K, van den Bosch RCE, Statler TS (2006) The black hole in NGC 3379: a comparison of gas and stellar dynamical mass measurements with HST and integral-field data. MNRAS 370(2):559-579. https://doi.org/10.1111/j.1365-2966. 2006.10537.x. arXiv:astro-ph/0605479

Shlosman I, Begelman MC, Frank J (1990) The fuelling of active galactic nuclei. Nature 345(6277):679-686. https://doi.org/10.1038/345679a0

Siegel MH, Dotter A, Majewski SR, Sarajedini A, Chaboyer B, Nidever DL, Anderson J, Marín-Franch A, Rosenberg A, Bedin LR, Aparicio A, King I, Piotto G, Reid IN (2007) The ACS survey of galactic globular clusters: M54 and young populations in the Sagittarius dwarf spheroidal galaxy. ApJ 667:L57-L60. https://doi.org/10.1086/522003. arXiv:arXiv:0708.0027

Silk J, Wyse RFG, Shields GA (1987) On the origin of dwarf galaxies. ApJ 322:L59. https://doi.org/10. $1086 / 185037$

Sills A, Dalessandro E, Cadelano M, Alfaro-Cuello M, Kruijssen JMD (2019) Light element variations within the different age-metallicity populations in the nucleus of the Sagittarius dwarf. MNRAS 490(1):L67-L70. https://doi.org/10.1093/mnrasl/slz149. arXiv:1910.00080

Spengler C, Côté P, Roediger J, Ferrarese L, Sánchez-Janssen R, Toloba E, Liu Y, Guhathakurta P, Cuillandre JC, Gwyn S, Zirm A, Muñoz R, Puzia T, Lançon A, Peng EW, Mei S, Powalka M (2017) Virgo redux: the masses and stellar content of nuclei in early-type galaxies from multiband photometry and spectroscopy. ApJ 849(1):55. https://doi.org/10.3847/1538-4357/aa8a78. arXiv: 1709.00406

Stone NC, Metzger BD (2016) Rates of stellar tidal disruption as probes of the supermassive black hole mass function. MNRAS 455:859-883. https://doi.org/10.1093/mnras/stv2281. arXiv:1410.7772

Stone NC, Küpper AHW, Ostriker JP (2017) Formation of massive black holes in galactic nuclei: runaway tidal encounters. MNRAS 467:4180-4199. https://doi.org/10.1093/mnras/stx097. arXiv: 1606.01909

Strader J, Seth AC, Forbes DA, Fabbiano G, Romanowsky AJ, Brodie JP, Conroy C, Caldwell N, Pota V, Usher C, Arnold JA (2013) The densest galaxy. ApJ 775(1):L6. https://doi.org/10.1088/2041-8205/ 775/1/L6. arXiv:1307.7707 
Strubbe LE, Quataert E (2009) Optical flares from the tidal disruption of stars by massive black holes. MNRAS 400(4):2070-2084. https://doi.org/10.1111/j.1365-2966.2009.15599.x. arXiv:0905.3735

Tonry JL (1984) Evidence for a central mass concentration in M 32. ApJ 283:L27-L30. https://doi.org/10. $1086 / 184326$

Tonry JL (1987) A central black hole in M32. ApJ 322:632. https://doi.org/10.1086/165758

Tremaine S (1995) An eccentric-disk model for the nucleus of M31. AJ 110:628. https://doi.org/10.1086/ 117548. arXiv:astro-ph/9502065

Tremaine S (2019) Order-disorder phase transition in black-hole star clusters. II. A scale-free cluster. MNRAS. https://doi.org/10.1093/mnras/stz3181

Tremaine SD, Ostriker JP, Spitzer L (1975) The formation of the nuclei of galaxies. I-M31. ApJ 196:407-411

Tremou E, Strader J, Chomiuk L, Shishkovsky L, Maccarone TJ, Miller-Jones JCA, Tudor V, Heinke CO, Sivakoff GR, Seth AC, Noyola E (2018) The MAVERIC survey: still no evidence for accreting intermediate-mass black holes in globular clusters. ApJ 862(1):16. https://doi.org/10.3847/15384357/aac9b9. arXiv:1806.00259

Tsatsi A, Mastrobuono-Battisti A, van de Ven G, Perets HB, Bianchini P, Neumayer N (2017) On the rotation of nuclear star clusters formed by cluster inspirals. MNRAS 464(3):3720-3727. https://doi. org/10.1093/mnras/stw2593. arXiv:1610.01162

Turner ML, Côté P, Ferrarese L, Jordán A, Blakeslee JP, Mei S, Peng EW, West MJ (2012) The ACS Fornax Cluster Survey. VI. The nuclei of early-type galaxies in the Fornax Cluster. ApJS 203:5. https://doi.org/10.1088/0067-0049/203/1/5. arXiv:1208.0338

Valluri M, Ferrarese L, Merritt D, Joseph CL (2005) The low end of the supermassive black hole mass function: constraining the mass of a nuclear black hole in NGC 205 via stellar kinematics. Astrophys J 628(1):137-152. https://doi.org/10.1086/430752. arXiv:astro-ph/0502493

van den Bergh S (1986) Nucleated spheroidal galaxies. AJ 91:271-274. https://doi.org/10.1086/114006

van den Bosch FC, Ogiya G, Hahn O, Burkert A (2018) Disruption of dark matter substructure: fact or fiction? MNRAS 474(3):3043-3066. https://doi.org/10.1093/mnras/stx2956. arXiv:1711.05276

van der Marel RP, Anderson J (2010) New limits on an intermediate-mass black hole in omega centauri. II. Dynamical models. ApJ 710(2):1063-1088. https://doi.org/10.1088/0004-637X/710/2/1063. arXiv:0905.0638

van der Marel RP, Evans NW, Rix HW, White SDM, de Zeeuw T (1994) Velocity profiles of galaxies with claimed black holes. II. $f\left(E, L_{z}\right)$ models for M32. MNRAS 271:99-117. https://doi.org/10.1093/ mnras/271.1.99

van Velzen S (2018) On the mass and luminosity functions of tidal disruption flares: rate suppression due to black hole event horizons. ApJ 852(2):72. https://doi.org/10.3847/1538-4357/aa998e. arXiv:1707. 03458

Vasiliev E (2017) A new Fokker-Planck approach for the relaxation-driven evolution of galactic nuclei. ApJ 848(1):10. https://doi.org/10.3847/1538-4357/aa8cc8. arXiv:1709.04467

Vasiliev E, Antonini F, Merritt D (2015) The final-parsec problem in the collisionless limit. ApJ 810(1):49. https://doi.org/10.1088/0004-637X/810/1/49. arXiv:1505.05480

Verolme EK, Cappellari M, Copin Y, van der Marel RP, Bacon R, Bureau M, Davies RL, Miller BM, de Zeeuw PT (2002) A SAURON study of M32: measuring the intrinsic flattening and the central black hole mass. MNRAS 335(3):517-525. https://doi.org/10.1046/j.1365-8711.2002.05664.x. arXiv: astro-ph/0201086

Villanova S, Geisler D, Gratton RG, Cassisi S (2014) The metallicity spread and the age-metallicity relation of $\omega$ Centauri. ApJ 791(2):107. https://doi.org/10.1088/0004-637X/791/2/107. arXiv:1406. 5069

Voggel K, Hilker M, Richtler T (2016) Globular cluster clustering and tidal features around ultra-compact dwarf galaxies in the halo of NGC 1399. A\&A 586:A102. https://doi.org/10.1051/0004-6361/ 201527070. arXiv: 1510.05010

Voggel KT, Seth AC, Neumayer N, Mieske S, Chilingarian I, Ahn C, Baumgardt H, Hilker M, Nguyen DD, Romanowsky AJ, Walsh JL, den Brok M, Strader J (2018) Upper limits on the presence of central massive black holes in two ultra-compact dwarf galaxies in Centaurus A. ApJ 858(1):20. https://doi.org/10.3847/1538-4357/aabae5. arXiv:1803.09750

Voggel KT, Seth AC, Baumgardt H, Mieske S, Pfeffer J, Rasskazov A (2019) The impact of stripped nuclei on the supermassive black hole number density in the local universe. ApJ 871(2):159. https:// doi.org/10.3847/1538-4357/aaf735 
Volonteri M (2010) Formation of supermassive black holes. A\&AR 18(3):279-315. https://doi.org/10. 1007/s00159-010-0029-x. arXiv:1003.4404

Volonteri M (2012) The formation and evolution of massive black holes. Science 337(6094):544. https:// doi.org/10.1126/science.1220843. arXiv:1208.1106

Walcher CJ, van der Marel RP, McLaughlin D, Rix HW, Böker T, Häring N, Ho LC, Sarzi M, Shields JC (2005) Masses of star clusters in the nuclei of bulgeless spiral galaxies. ApJ 618:237-246. https:// doi.org/10.1086/425977. arXiv:astro-ph/0409216

Walcher CJ, Böker T, Charlot S, Ho LC, Rix HW, Rossa J, Shields JC, van der Marel RP (2006) Stellar populations in the nuclei of late-type spiral galaxies. ApJ 649:692-708. https://doi.org/10.1086/ 505166. arXiv:astro-ph/0604138

Walker MF (1964) Two-color composite photographs of M 33. AJ 69:744. https://doi.org/10.1086/ 109350

Wehner EH, Harris WE (2006) From supermassive black holes to dwarf elliptical nuclei: a mass continuum. ApJ 644(1):L17-L20. https://doi.org/10.1086/505387. arXiv:astro-ph/0603801

Wevers T, van Velzen S, Jonker PG, Stone NC, Hung T, Onori F, Gezari S, Blagorodnova N (2017) Black hole masses of tidal disruption event host galaxies. MNRAS 471(2):1694-1708. https://doi.org/10. 1093/mnras/stx1703. arXiv:1706.08965

Wevers T, Stone NC, van Velzen S, Jonker PG, Hung T, Auchettl K, Gezari S, Onori F, Mata Sánchez D, Kostrzewa-Rutkowska Z, Casares J (2019) Black hole masses of tidal disruption event host galaxies II. MNRAS 487(3):4136-4152. https://doi.org/10.1093/mnras/stz1602. arXiv:1902.04077

Williams BF, Dolphin AE, Dalcanton JJ, Weisz DR, Bell EF, Lewis AR, Rosenfield P, Choi Y, Skillman E, Monachesi A (2017) PHAT. XIX. The ancient star formation history of the M31 disk. ApJ 846(2):145. https://doi.org/10.3847/1538-4357/aa862a

Wise JH, Regan JA, O'Shea BW, Norman ML, Downes TP, Xu H (2019) Formation of massive black holes in rapidly growing pre-galactic gas clouds. Nature 566(7742):85-88. https://doi.org/10.1038/ s41586-019-0873-4. arXiv:1901.07563

Woo JH, Cho H, Gallo E, Hodges-Kluck E, Le HAN, Shin J, Son D, Horst JC (2019) A 10,000-solar-mass black hole in the nucleus of a bulgeless dwarf galaxy. Nat Astron 3:755-759. https://doi.org/10. 1038/s41550-019-0790-3. arXiv:1905.00145

Worthey G (1994) Comprehensive stellar population models and the disentanglement of age and metallicity effects. ApJS 95:107. https://doi.org/10.1086/192096

Zhang F, Shao L, Zhu W (2019) Gravitational-wave merging events from the dynamics of stellar-mass binary black holes around the massive black hole in a galactic nucleus. ApJ 877(2):87. https://doi. org/10.3847/1538-4357/ab1b28. arXiv:1903.02685

Publisher's Note Springer Nature remains neutral with regard to jurisdictional claims in published maps and institutional affiliations. 